University of Rhode Island

DigitalCommons@URI

Open Access Dissertations

2019

\title{
ADVANCEMENTS IN BIOMIMETIC UNDERWATER PROPULSION: BOUNDARY INTERACTIONS AND CROSSFLOW COMPENSATION
}

Matthew Perkins

University of Rhode Island, perkinsm204@gmail.com

Follow this and additional works at: https://digitalcommons.uri.edu/oa_diss

\section{Recommended Citation}

Perkins, Matthew, "ADVANCEMENTS IN BIOMIMETIC UNDERWATER PROPULSION: BOUNDARY INTERACTIONS AND CROSSFLOW COMPENSATION" (2019). Open Access Dissertations. Paper 963. https://digitalcommons.uri.edu/oa_diss/963

This Dissertation is brought to you for free and open access by DigitalCommons@URI. It has been accepted for inclusion in Open Access Dissertations by an authorized administrator of DigitalCommons@URI. For more information, please contact digitalcommons-group@uri.edu. 


\section{ADVANCEMENTS IN BIOMIMETIC UNDERWATER PROPULSION: BOUNDARY INTERACTIONS AND CROSSFLOW COMPENSATION \\ BY \\ MATTHEW PERKINS}

A DISSERTATION SUBMITTED IN PARTIAL FULFILLMENT OF THE REQUIREMENTS FOR THE DEGREE OF DOCTOR OF PHILOSOPHY

IN

OCEAN ENGINEERING 


\title{
DOCTOR OF PHILOSOPHY DISSERTATION
}

$\mathrm{OF}$

MATTHEW PERKINS

\section{APPROVED:}

Dissertation Committee:

\author{
Major Professor Stephen C. Licht \\ Jason M. Dahl \\ Richard J. Vaccaro \\ David N. Beal \\ Nasser H. Zawia \\ DEAN OF THE GRADUATE SCHOOL
}

UNIVERSITY OF RHODE ISLAND

2019 


\begin{abstract}
Biomimetic propulsion is an active area of research in the field of robotics. This work presents advancements to help improve the performance of vehicles employing oscillating foil propulsion. For vehicles operating near the sea floor, ground effect on rolling and pitching foils was investigated in Manuscript 1. The 'ground effect' was found to be present and to cause a repulsion force on the foil as well as an increased thrust force. It was also shown that ground effect can be activated by biasing the foil into the ground effect zone while the vehicle maintains a greater distance from the boundary. The effects on the foil due to 'ground effect' were compared to previous work done with heaving and pitching foils showing that 'ground effect' is an inherently 3 dimensional phenomena and studies using 2 dimensional constrained flow cannot be extrapolated into rolling and pitching foils. The second manuscript expands on this work, investigating the effect that free surface has on the oscillating foil. Similar to 'ground effect' it was found that the presence of the boundary creates a repulsive force pushing the foil away from the boundary. However, instead of increased thrust, the free surface causes a decrease in thrust forces on the foil. The third manuscript uses an analytical model to design a control system that uses force feedback from the foil in order to compensate and estimate a constant disturbance such as crossflow. The control system was able to compensate for a constant crossflow and was able to estimate the angle of the crossflow based on the force feedback to within 0.5 degrees. These advancements will help improve vehicles with oscillating foil propulsion that will open up new opportunities for investigation and exploration of complex oceanic environments.
\end{abstract}




\section{ACKNOWLEDGMENTS}

I am extremely grateful for all of the support I have gotten over these many years. I would like to personally thank Stephen Licht for taking me on as a graduate student so many years ago. Over the past 5 years I have learned a lot from him and it was because of him that I've had this great opportunity to obtain my doctorate. I would like to thank all my friends and family for the love and support that they have lent me through the low times and the highs. I'm very excited to start the next stage of my life and am happy to have had such support along the way. Thank you all. 


\section{TABLE OF CONTENTS}

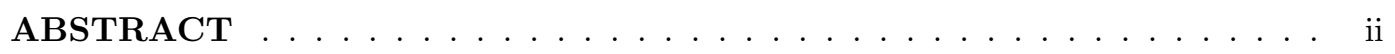

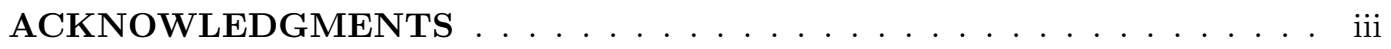

TABLE OF CONTENTS $\ldots \ldots \ldots \ldots \ldots \ldots \ldots$ iv

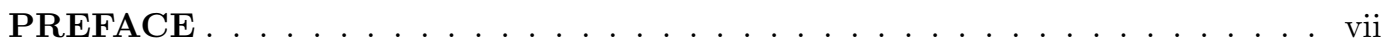

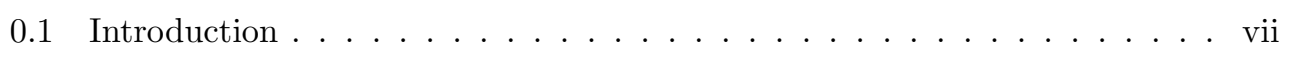

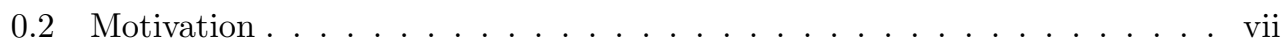

0.3 Manuscript Contributions . . . . . . . . . . . . viii

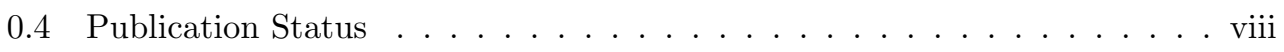

\section{MANUSCRIPT}

1 Rolling and pitching oscillating foil propulsion in ground effect. . . . 1

1.1 Introduction and Background . . . . . . . . . . . . 1

1.1.1 1-D Pitching or Heaving only Foils . . . . . . . . . . . . 2

1.1.2 2-D Combined Heaving and Pitching Foils . . . . . . . . . . . 2

1.1.3 3-D Rolling and Pitching Foils .............. 3

1.2 Methodology . . . . . . . . . . . . . . . . . . 3

1.2.1 Experimental Apparatus . . . . . . . . . . . . . . . 4

$1.2 .2 \quad$ Experimental Procedure . . . . . . . . . . . . . . . . . 12

1.3 Results . . . . . . . . . . . . . . . . . . . . 13

1.3.1 Evolution of $\overline{C_{L}}$ with transition into ground effect test zone. . . . . 14

1.3.2 Variation in mean thrust . . . . . . . . . . . . . . . . . 16

1.3.3 Change in lift and thrust in response to changes in proximity and foil orientation. . . . . . . . . . . . . . 18

1.3.4 Comparison to change in lift with two dimensional foil kinematics. 18

1.3.5 Cross platform comparison across a range of kinematic parameters. 21

1.4 Discussion . . . . . . . . . . . . . . . . 23 
1.4.1 Foils used to generate high thrust with rolling and pitching oscillations experience a 'ground effect' which repels them from solid boundaries. . . . . . . . . . . . . . . . 23

1.4.2 Ground effect can be induced at greater distances by biasing the tip of the foil toward the boundary. . . . . . . . . . 24

1.4.3 The ground effect experienced by a high-aspect ratio rolling and pitching foil is a fully three-dimensional phenomenon, which cannot be accurately predicted when two-dimensional flow and/or two-dimensional kinematics are enforced. . . . . . . . 24

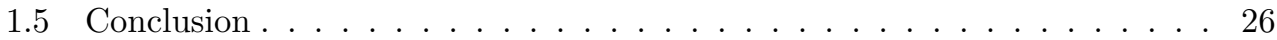

List of References . . . . . . . . . . . . . . . . . . . . . . . 27

2 Free surface boundary effects on rolling and pitching oscillating foil

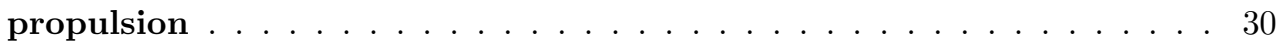

2.1 Introduction and Background $\ldots \ldots \ldots 30$

2.1 .1 Oscillating Foils . . . . . . . . . . . . . . 30

2.1 .2 Effect of the boundary . . . . . . . . . . . . . 31

2.1 .3 Contributions . . . . . . . . . . . . . . . . . 32

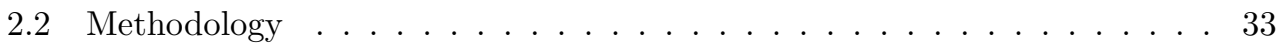

$2.2 .1 \quad$ Experimental Apparatus . . . . . . . . . . . . . . . . 33

$2.2 .2 \quad$ Experimental Procedure . . . . . . . . . . . . . . . . . 38

2.3 Results . . . . . . . . . . . . . . . . . . . . . 39

2.3 .1 Mean thrust in open water f . . . . . . . . . . . . 40

2.3 .2 Change in mean lift $\ldots \ldots \ldots \ldots \ldots$

2.3 .3 Changes in mean thrust . . . . . . . . . . . . . . . . 41

2.3.4 Boundary effect comparison across a range of kinematic parameters. 43

2.4 Discussion . . . . . . . . . . . . . . . . . . . 44

2.4.1 Thrust generating rolling and pitching oscillating foils experience a repulsion force as they approach a free surface. . . . . . . . . 44

2.4.2 Observed effect on lift forces of a foil oscillating are similar for free surface and solid boundaries. . . . . . . . . . . . . 44

2.4.3 The difference between ground effect and surface effect on thrust production can be accounted for by wave generation. . . . . . 45 


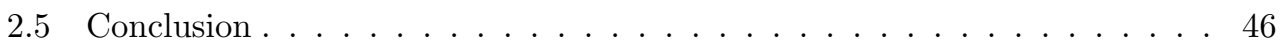

List of References . . . . . . . . . . . . . . . . . . . . 47

3 Combination of force feedback and an analytic flapping foil model to improve control of a flapping foils in the presence of crossflow . . . . . 49

3.1 Introduction and Background . . . . . . . . . . . . . . . 49

$3.1 .1 \quad$ Oscillating Foils . . . . . . . . . . . . . . . . . . . . . . . 49

3.1 .2 Digital Control Theory . . . . . . . . . . . . . . . . . . 50

3.1 .3 Contributions . . . . . . . . . . . . . . . . . . 51

$3.1 .4 \quad$ Useful Definitions . . . . . . . . . . . . . . . . . . . . . 52

3.2 Model Implementation . . . . . . . . . . . . . . . . . . . . . 52

3.2 .1 Result Replication . . . . . . . . . . . . . . . . 55

3.3 Evaluating Model Accuracy . . . . . . . . . . . . . . . . 56

3.3 .1 Experimental Apparatuses . . . . . . . . . . . . . . . . . 57

3.3.2 3-D rolling and pitching foil model validation . . . . . . . . 63

3.4 Using Analytic Model as a Design Tool . . . . . . . . . . . . . . . . . 69

3.4.1 System Model . . . . . . . . . . . . . . . . . . 69

3.4 .2 Gain Calculation and Simulation . . . . . . . . . . . 70

3.4.3 Crossflow Compensation using force feedback . . . . . . . . . . 72

3.5 Discussion . . . . . . . . . . . . . . . . . . 75

3.5.1 The code based on the analytical model provides accurate force prediction across a range of foil configurations, kinematics, and environmental factors. . . . . . . . . . . . . 76

3.5.2 A simple control system combined with an analytical model can compensate for and estimate crossflow. . . . . . . . . . 76

3.5.3 This model is a valid design tool for designing control systems of 3-D rolling and pitching oscillating foils . . . . . . 77

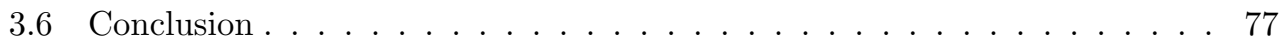

3.7 Future Work . . . . . . . . . . . . . . . . 78

List of References . . . . . . . . . . . . . . . . . . . 78

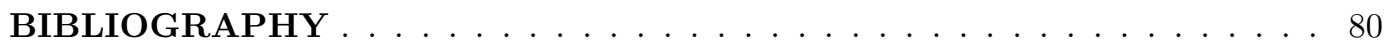




\section{PREFACE}

\subsection{Introduction}

Unmanned underwater vehicles (UUVs) have a proven track record as reliable, versatile platforms for scientific, commercial, or military applications. Current UUV technology does, however, have its limitations. Conventional UUV's are torpedo shaped bodies, with a propeller and lifting surfaces for control. While effective in calm waters, they do not perform well in areas of more dynamic flow, nor in near bottom or complex environments. Access to these areas is essential for the furthering study of nearshore ocean dynamics, removal of unexploded ordinance and countless other applications. This has led to an increase in biomimetics and bio-inspired engineering in order to develop novel ideas for new generations of UUVs.

\subsection{Motivation}

Complex underwater environments are difficult to navigate through, hindering efforts of robotics to operate in these areas. Turtle-like robots emulate the locomotion of turtles with oscillating(flapping) foils inspired by the fins and motions of a sea turtle. Oscillating foils provide vehicles with advantages such as increased low-speed maneuverability and agility. Vehicles equipped with this type of propulsion have a wide range of applications, both scientific and militaristic. I believe that vehicles with biomimetic propulsion are the next technological step in autonomous vehicles. There are many different styles of flapping foils, however, most vehicles use rolling and pitching coupled motions since they are easier to implement in an autonomous vehicle. For this reason my research aims to improve the understanding of this type of system. Vehicles with greater low-speed maneuverability will allow autonomous systems to investigate more complex environments. My research aims to shed light upon the performance of these oscillating foil systems in the presence of different types of boundaries and also in the presence of a crossflow. Understanding how these environments affect the forces on the foil will allow us to improve the performance of vehicles operating in near-surface and near-bottom conditions. Crossflows are also a common occurrence due to ocean currents, however, it could also be a crossflow caused by the vehicle traveling upwards or downwards while simultaneously moving forward. Furthermore, advanced spinning maneuvers would also create more complex flow about the fin. 


\subsection{Manuscript Contributions}

This dissertation is made up of three separate but related manuscripts. Manuscript 1 studies an oscillating foil operating near a solid boundary such as the sea floor where there is expected to be a 'ground effect zone.' This is a zone close to the boundary that affects the lift and thrust on a wing or foil. The effect has been studied extensively in aerodynamics as well as with other types of oscillating foil systems. Manuscript 1 describes the appearance and magnitude of this effect as well as comparing the effect to that seen on other system configurations. Manuscript 2 extends the work of Manuscript 1 to the effect that free-surface such as the ocean surface has on the oscillating foil. The effect is detailed in this work as well as being compared to results from the work on 'ground effect.' Finally, Manuscript 3 employs a new analytic model to design a control system that effectively compensates for and estimates the angle of a crossflow using force feedback from the foil. All of this work will help improve the performance of these biomimetic vehicles, providing better platforms to investigate and explore the oceans.

\subsection{Publication Status}

Manuscript 1 has already been published in Bioinspriation and Biomimetics. Manuscript 2 will be submitted for review in the future. Manuscript 3 may be submitted for review in the future. 


\section{MANUSCRIPT 1}

\section{Rolling and pitching oscillating foil propulsion in ground effect.}

In this paper, we investigate the effect of operating near a solid boundary on the forces produced by harmonically oscillating thrust generating foils. A rolling and pitching foil was towed in a freshwater tank in a series of experiments with varying kinematics. Hydrodynamic forces and torques were measured in the freestream and at varying distances from a solid boundary, and changes in mean lift and thrust were found when the foil approached the boundary. The magnitude of this ground effect exhibited a strong nonlinear dependence on the distance between the foil and the boundary. Significant effects were found within three chord lengths of the boundary, and ground effect can be induced at greater distances from the boundary by biasing the tip of the foil toward the boundary. Lift coefficients changed by as much as 0.2 at the closest approach to the ground, with changes $>0.05$ for all cases across Strouhal number ranging from 0.3 to 0.6 , and nominal maximum angle of attack ranging from $20^{\circ}$ to $40^{\circ}$. The ubiquity of the ground effect in high thrust kinematics suggests that the ground effect can provide a passive obstacle avoidance capability for foil propelled vehicles. By comparison to previous experimental work, we find that the ground effect experienced by a high-aspect ratio rolling and pitching foil is a fully three-dimensional phenomenon, as it is not accurately predicted when two-dimensional flow

and/or two-dimensional kinematics are enforced. While two dimensional foil kinematics are more easily modeled for numerical studies, three-dimensional foil kinematics may be more practical for real world implementation in underwater vehicles.

\subsection{Introduction and Background}

The complexity of operating efficiently and effectively underwater has driven researchers and engineers to search for biological examples to improve propulsion mechanisms in underwater vehicles. In this paper, we focus on the use of harmonically oscillating foils in the presence of obstacles. For discussion, we can categorize oscillating foils by the number of degrees of freedom in the driving motion of the foil. Three propulsive configurations for rigid foils have been extensively studied: one-dimensional (pitching or heaving only), two-dimensional (combined pitching and heaving motions), and three-dimensional (coupled rolling and pitching motions). The effect of operating near a solid bottom boundary, i.e. 
in 'ground effect', has been investigated in the first two foil configurations. This paper is a first experimental study of three dimensional high aspect ratio thrust producing foils in ground effect.

\subsubsection{1-D Pitching or Heaving only Foils}

Investigations of foils that oscillate about a single axis or in a single direction include studies of pitching-only and heaving-only motion. 'Pitching only' foils rotate about an axis parallel to the foil span. 'Heaving only' foils maintain a constant foil orientation while the foil is oscillated in a direction perpendicular to the incoming flow. Early visualization efforts demonstrated that both the pitching motion [1] and the heaving motion alone [2] can produce thrust through an alternating vortex wake structure. The wake structure depends on Strouhal number and maximum angle of attack. The dynamics of both modes of operation in the absence of obstacles have since been extensively studied.

Numerical studies with heaving only foils in the presence of a solid boundary [3] predict that a foil can experience either net suction or repulsion force, depending on the distance between the foil and the boundary. [4] found that self-propelled rigid foils in the presence of a wall will yield increased cruising speed with a reduced efficiency if the kinematics are unchanged. Flexible foils however will experience both increased speed and efficiency. Numerical simulations by [5] and [6] have also found increased thrust by propulsors near a wall. Flow tank experiments with pitching only foils find that rectangular foils undergoing pitch oscillations generate higher thrust when near a boundary [7] and that a suction region exists very near the wall with a repulsion region at moderate distances from the wall [8]. Using a very flexible self propelled foil actuated in pitch [9] showed that the wall affects the jet structure of the wake, enhancing the thrust and altering the lift force when compared to freestream operation.

\subsubsection{2-D Combined Heaving and Pitching Foils}

Combining heave and pitch motions while enforcing two-dimensional flow has allowed investigators to more closely approximate real animal mechanisms while limiting system complexity, particularly in measurement and analysis of fluid flow. A wide variety of kinematic and structural parameters have been investigated experimentally with heaving and pitching foils. [10] performed experimental studies of the forces as well as flow visualization of the wake. The test apparatus included restrictor plates attached to the sides of the foil 
to reduce spanwise flow around the tips, thus minimizing three dimensional effects. Wake structure behind the foil was parameterized based on maximum angle of attack and Strouhal number. [11] found that there was a plateau of 50\%-60\% propulsive efficiency in a range of Strouhal numbers from 0.05 to 0.6 with maximum angle ranging from $10^{\circ}$ to $50^{\circ}$ and that by adding a bias to the mean foil pitch angle, effective force vectoring is possible. [12] found that variations in chordwise flexibility can be used to increase propulsive efficiency. In experiments where flow around the foil tip was allowed, [13] and [14], found that adding in-line motion with a power/feathering stroke style provides substantial improvements on force vectoring without reducing propulsive efficiency.

Operation of pitching and heaving foils near a boundary was investigated in [15] and [16] for a flexible undulating foil with flow around both ends. In [17] and [8], ground effect is treated as a 2 dimensional flow phenomenon, but [18] suggests that ground effect is inherently three dimensional as flow around the tip of a foil has a strong effect on the hydrodynamic forcing near the boundary. Out of plane flow around the tip of the foil caused significant differences in both the magnitude and direction of the ground effect, when compared to the case with purely two dimensional flow.

\subsubsection{3-D Rolling and Pitching Foils}

For marine animals, fin propulsion is an inherently three dimensional phenomenon, both in terms of the motion of the foil and the resulting fluid flow. We classify as three dimensional foils those that use a rotary oscillation about a shoulder joint, instead of linear heaving motion, coupled with oscillatory pitching. This configuration is more appropriate for integration into underwater vehicles as a propulsion method [19]. For three dimensional foils, [20] and [21] found similar dependencies of lift and thrust coefficients on Strouhal number and maximum angle of attack as in two dimensional foils. The influence of a solid boundary has been extensively studied for one and two dimensional foils. In the current work, we extend our investigation of oscillating foil ground effect to encompass fully three dimensional geometry and flow during operation near the boundary. To our knowledge this is the first experimental study of ground effect in rolling and pitching foils.

\section{$1.2 \quad$ Methodology}

A rolling and pitching foil was oscillated while towed in a freshwater tank, in a series of experiments with varying foil kinematics. The instantaneous fluid forces and mo- 
ments applied to the foil were recorded as the foil moved from a constant depth deep water ('freestream') section, through a 'transition' section in which the tank depth linearly decreased, into a constant depth shallow water ('ground effect') test section.

\subsubsection{Experimental Apparatus}

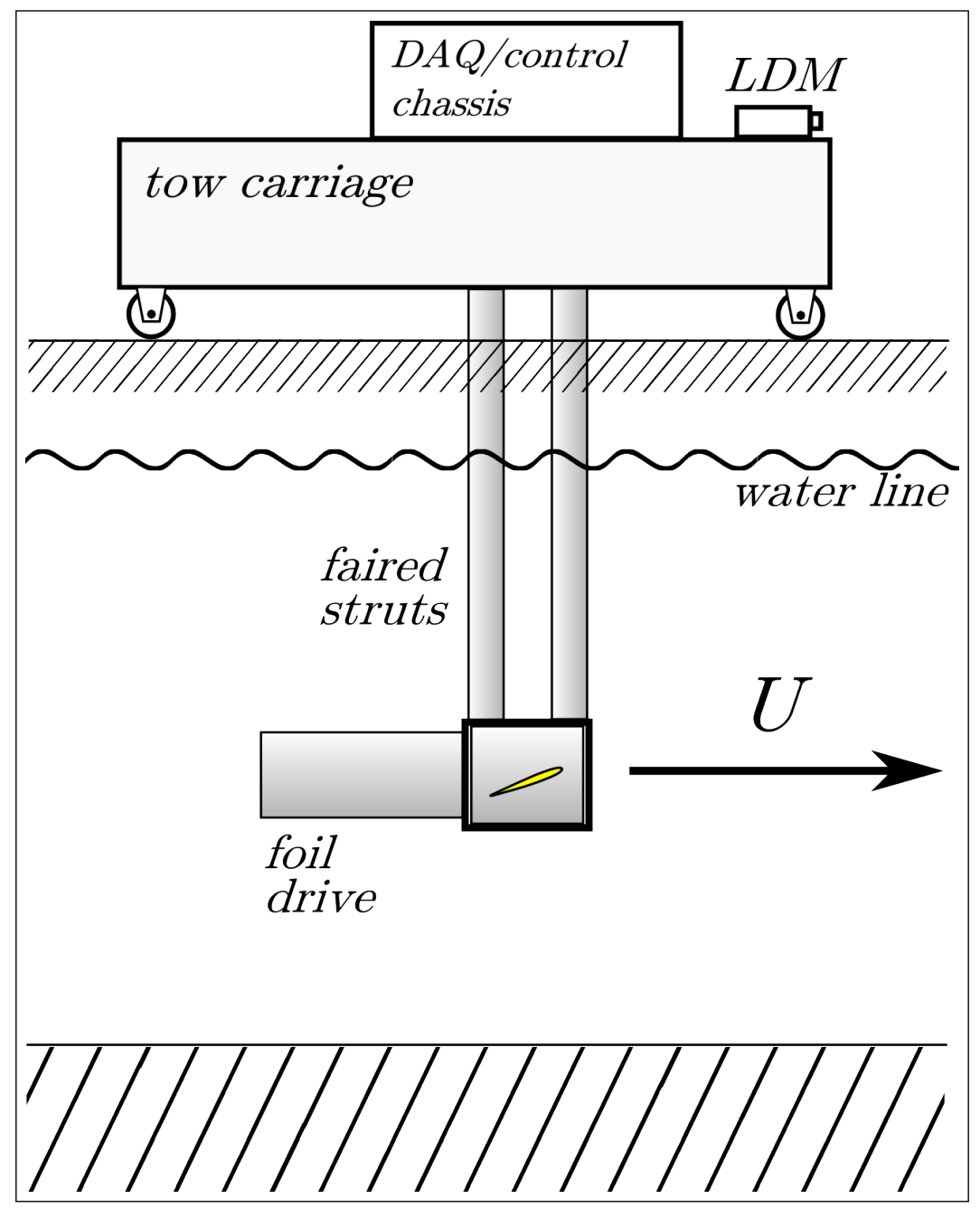

Figure 1.2.1: Side view of foil apparatus as installed in tow tank. DAQ, communications, and foil control are managed through an integrated electronics chassis mounted on the self-propelled tow carriage. The laser distance measurement (LDM) provides real-time measurement of carriage position relative to the end of the tow tank. The roll axis of the foil is parallel to the direction of motion.

The foil drive mechanism is mounted to the overhead towing carriage using a trio of faired struts, as shown in Figure 1.2.3 and described in [22]. The test apparatus is 


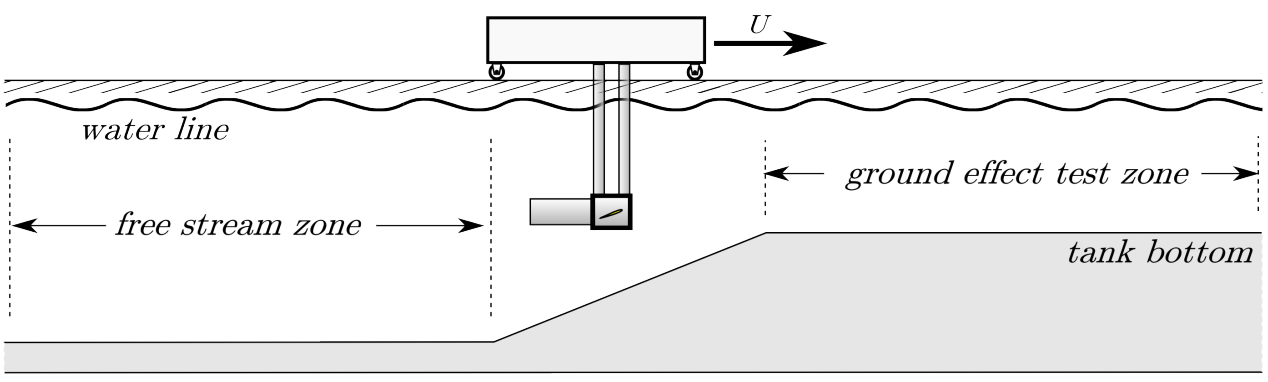

Figure 1.2.2: Side view cross section of the tow tank showing the depth profile created by adjustment of the tank's solid bottom panels. The foil drive is shown in the transition zone between freestream and ground effect test zone, and is shown mounted at a height which results in the closest bottom approach.

suspended such that the foil is centered in a water tank at a depth of $0.7 \mathrm{~m}$ (Figure 1.2.1). This motorized tow carriage travels the length of the tank by rolling on top of the tank walls. The tank is $3.65 \mathrm{~m}$ wide, with a total length of $30 \mathrm{~m}$ and maximum depth of $1.55 \mathrm{~m}$. The distances between the fin apparatus and the boundaries of the tank are equal to or greater than that used in foundational work on flapping foils, with similar chord lengths and Reynolds number, by [11] and [21] using two dimensional and three dimensional foils, respectively. The tank depth profile is defined using seven adjustable $2.43 \mathrm{~m}$ long bottom panels spanning the width of the tank. The depth profile used in this effort is shown to scale in Figure 1.2.2. The usable length for these tests consists of a 9 meter long 'freestream' zone, followed by a single panel 'transition' zone, and a 4 meter long 'ground effect' test zone. In the freestream zone, the foil is assumed to have no significant wake boundary interactions with the bottom, side walls, or free surface. The transition from freestream to the ground effect test zone occurs above a single sloped bottom panel. Using this configuration, we are able to directly compare measured forces in the freestream to those in the ground effect test zones during a single experimental trial, minimizing the effect of run to run discrepancies such as small non-zero pitch biases or misalignments of the entire apparatus.

\subsubsection{Foil Drive Mechanism}

The foil oscillation is actuated by two $24 \mathrm{~V}$ DC brush servo motors (LittonPolyscientific), each mounted inside a pressure housing. The rolling motion is actuated using a $196 \mathrm{~W}$ motor with a 25:1 gearing contained in a sealed PVC cylinder, the 'roll canister'. The output shaft of the roll actuation motor protrudes through a dynamic o-ring seal to drive a Delrin cylinder that is constrained at the opposite end with a plain rotary 


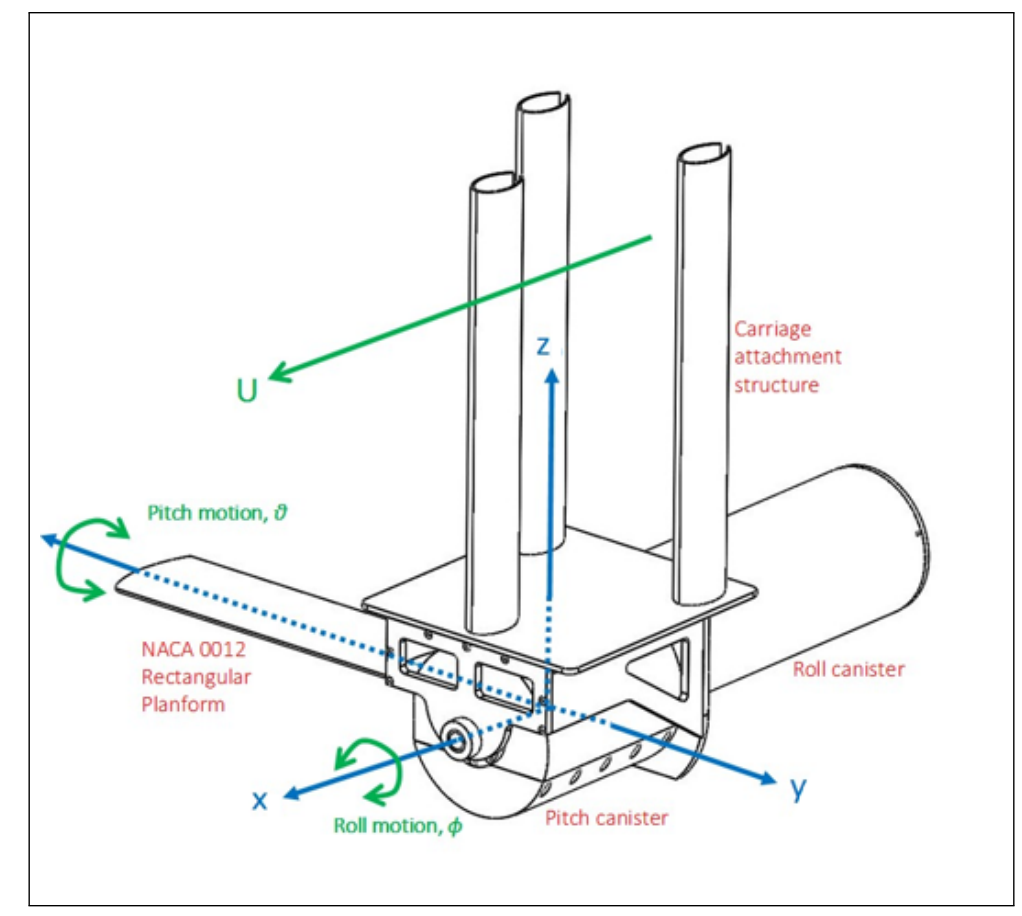

Figure 1.2.3: Foil drive mechanism and attachment. The submersible foil drive apparatus consists of two pressure housings, each containing a DC brush motor to actuate roll or pitch. Drive electronics are contained within the roll canister, while power and communication are supplied from the overhead carriage through underwater connectors.

The foil shaft bearings are mounted to a rigid platform supported by a 6 -DOF dynamometer inside the pitch canister, as detailed in Figure 1.2.5.

bearing. This 'pitch' canister is machined with an internal pocket which contains a $15 \mathrm{~W}$ motor with an 85:1 gearing for actuation of the pitching motion. Low level motor control is accomplished with a programmable two-axis motion control card (Galil 1425) and two PWM motor drives (Advanced Motion Devices) housed in the roll canister. Feedback from encoders, mounted on the drive motor shafts, to the dedicated motion control processor is used to ensure repeatable and accurate kinematics. The motion control processor is programmed to halt the motion if deviations of greater than two degrees from the desired kinematics occurs on either axis. The entire apparatus is a modified and updated version of a foil drive removed from the underwater vehicle described in [23]. The foil drive was augmented to enable direct measurement of the forces applied to the foil as described in [24]. 


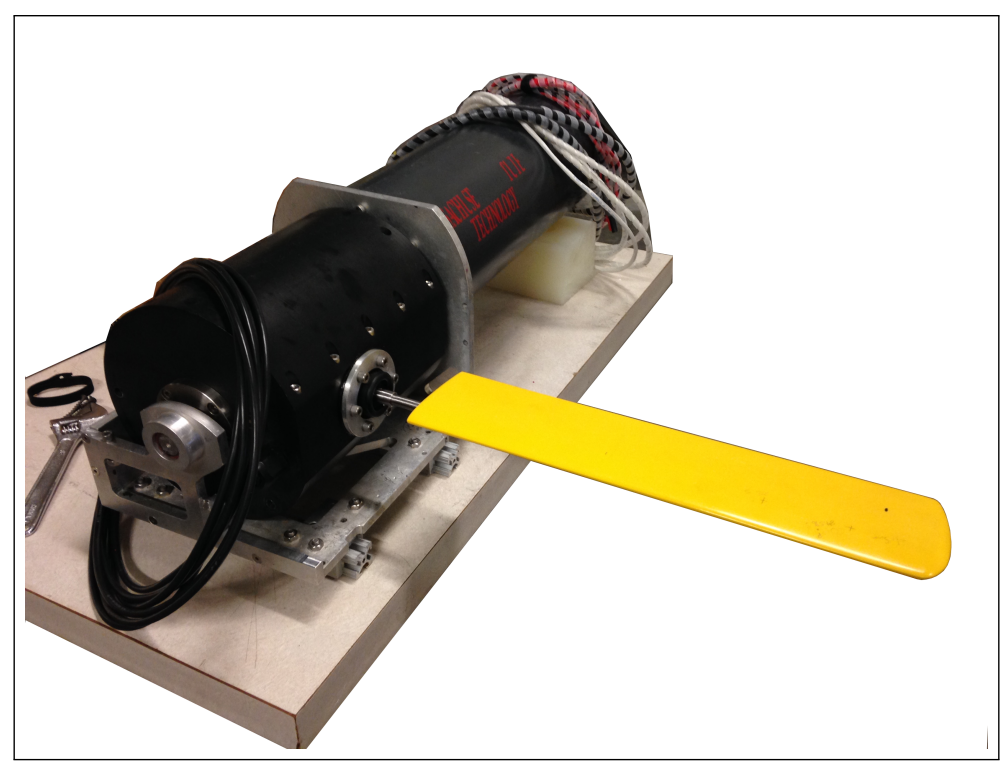

Figure 1.2.4: Image of foil drive apparatus prior to installation with foil mounted in place.

The foil has a uniform NACA-0012 cross-section, with span of $0.398 \mathrm{~m}$ and chord of $0.095 \mathrm{~m}$, and is nominally rigid, with a titanium frame and cast outer shape.

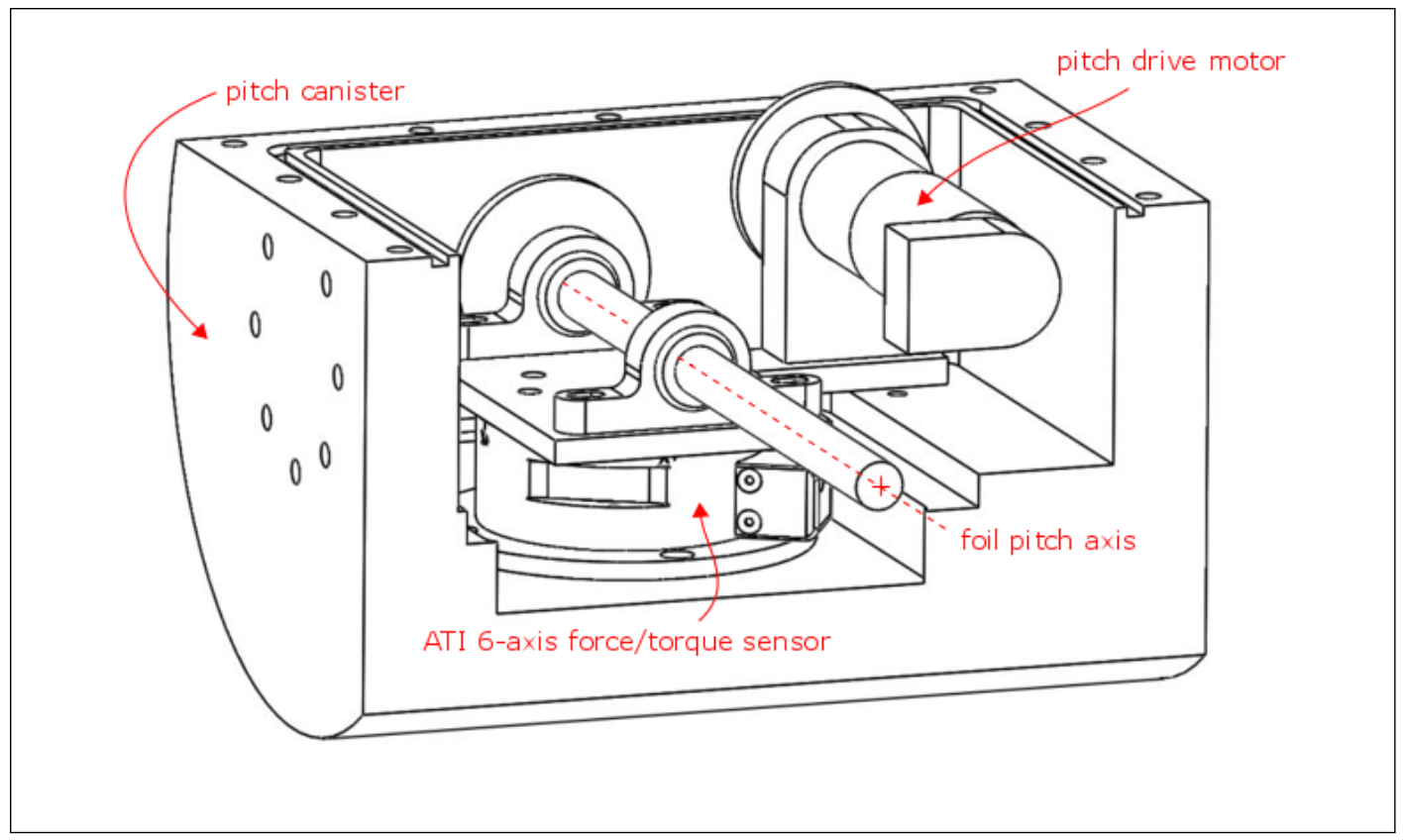

Figure 1.2.5: The pitch canister containing sensor and foil drive train. The front face of the canister is cut away in this view to show internal detail. The motor and shaft bearings are mounted to a rigid platform supported by the six-axis dynamometer. The shaft is driven by a chain drive with a one to one gear ratio, as shown in Figure 1.2.6. 


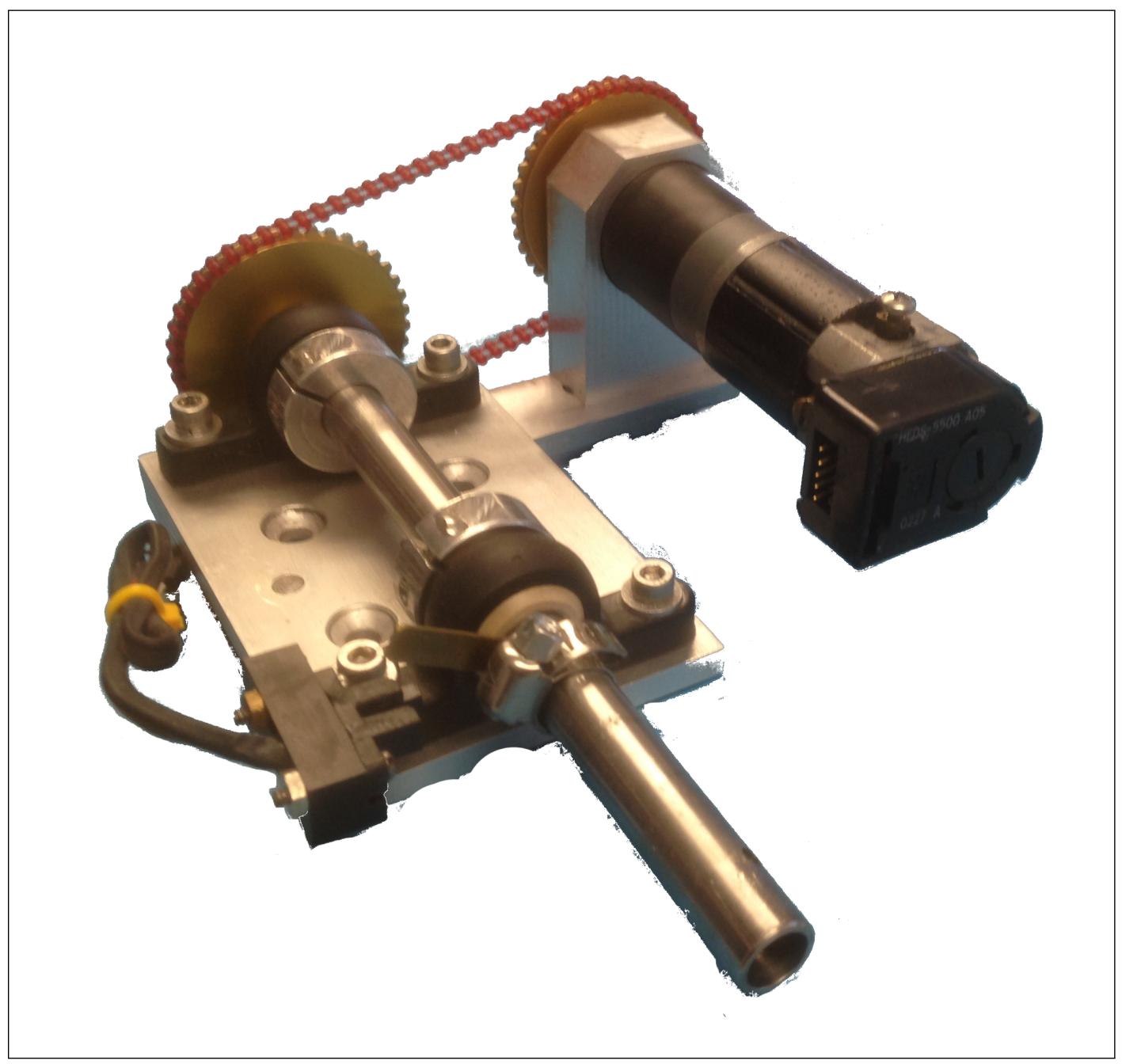

Figure 1.2.6: Pitch drive train. The foil shaft is connected to the gearbox output shaft using a chain drive.

\subsubsection{Sensor and Foil}

The test foil is a nominally rigid rectangular planform foil with a constant NACA-0012 cross section. It is constructed of a titanium framework cast inside resin with hardness Shore D 58 (Smooth-On Feather Lite), as shown in Figure 1.2.4. The foil has a span of $0.398 \mathrm{~m}$ and a chord of $0.095 \mathrm{~m}$ which yields an aspect ratio of 4.2 .

The foil attachment within the pitch canister is shown in cutaway detail in Figure 1.2.5. The pitch motor drive system and the foil shaft bearings are mounted directly to a 6 -axis strain-gauge dynamometer (ATI Gamma SI-65-5) installed inside the pitch canister. The foil is mounted directly to the sensor to avoid direct measurement of drag forces on the 
drive apparatus. Flow interaction between the drive apparatus and the foil is assumed to be a secondary effect. As the external geometry of the drive apparatus is identical to that of the free swimming vehicle in [23], it provides an accurate representation of a real world application. A floating dynamic o-ring seal, as detailed in [20], is used to minimize mechanical coupling between the canister and the foil shaft where it exits the housing.

The six analog outputs from the dynamometer strain gauges are amplified and then acquired using a 16-bit multiplexing DAQ module (National Instrument PXI-6225) installed in a carriage mounted chassis (National Instruments PXIe-1082). The linearity of the dynamometer was verified using hanging calibration weights applied to activate both positive and negative directions on all six axes. Five values were applied across the entire nominal measurement range for each axis and direction. The factory calibration was accurate to within $0.2 \%$ of the expected value for all weights applied. Nominal sensor resolution of $0.99 \mathrm{mN}$ in the $\mathrm{x}$ and $\mathrm{y}$ axes, $3.05 \mathrm{mN}$ in the $\mathrm{z}$-axis and $0.076 \mathrm{mN}-\mathrm{m}$ in all torque axes is achieved using the 16-bit analog to digital conversion. The angular position in roll is captured using a string potentiometer wrapped once around the moving canister.

Roll position is used to synchronize roll foil motion with the force data; the analog potentiometer output is acquired using the same DAQ module that acquires the dynamometer output. Carriage position relative to the tank is acquired at $10 \mathrm{~Hz}$ using a laser distance measurement device (Astech LDM42A) mounted to the carriage. All communication between the automated experimental script (implemented in LabView 7.0) on the operator workstation, and the sensors and actuators is routed through the carriage mounted chassis via a fiber optic link. 


\subsubsection{Foil Kinematics}

Table 1.2.1: Experimental Parameters: Constants

\begin{tabular}{ccc}
\hline Carriage velocity & $U$ & $0.5 \frac{\mathrm{m}}{\mathrm{s}}$ \\
Roll amplitude & $\Phi_{0}$ & $12^{\circ}$ \\
Foil span length & $\mathrm{S}$ & $0.398 \mathrm{~m}$ \\
Foil chord length & $\mathrm{c}$ & $0.095 \mathrm{~m}$ \\
Foil planform area & $A_{p}=S c$ & $0.038 \mathrm{~m}^{2}$ \\
Foil aspect ratio & $A R=\frac{S}{c}$ & 4.2 \\
Distance to 70\% span & $r_{0.7}$ & $0.41 \mathrm{~m}$ \\
2-D heave of 70\% span point & $h_{0.7}$ & $0.17 \mathrm{~m}$ \\
Kinematic viscosity, $\mathrm{H}_{2} \mathrm{O}$ & $\nu$ & $10^{-6}$ \\
Reynolds number & $\frac{U c}{\nu}$ & 57,000 \\
\hline
\end{tabular}

Table 1.2.2: Experimental Parameters: Independent Variables

\begin{tabular}{lcc}
\hline Max. nominal angle of attack & $\alpha_{\max }$ & {$[20,25,30,35,40]^{\circ}$} \\
Strouhal number & $S t$ & {$[0.3,0.4,0.5,0.6]$} \\
Heave to chord ratio & $\frac{h_{0.7}}{c}$ & 1.8 \\
$\begin{array}{l}\text { Height of pitch axis to chord ratio } \\
\text { with no } \Phi_{\text {bias }}\end{array}$ & $H^{*}=\frac{d}{c}$ & {$[1.4,1.65,1.9,2.15,2.4,2.65,2.9,3.4,3.9]$} \\
Downward roll bias $\left[^{\circ}\right]$ & & \\
\hline
\end{tabular}

Table 1.2.3: Experimental Parameters: Dependent Variables

\begin{tabular}{ccc}
\hline Pitch amplitude & $\theta_{0}$ & chosen to obtain desired $\alpha_{0}$ \\
Oscillation frequency & $\omega$ & chosen to obtain desired $S t$ \\
Mean roll bias & $\Phi_{\text {bias }}$ & chosen to obtain desired tip clearance \\
Height & $d$ & chosen to obtain desired $H^{*}$ \\
\hline
\end{tabular}

The foil kinematics in all experiments performed are completely be described by (1.1) and (1.2),

$$
\begin{gathered}
\Phi(t)=\Phi_{0} \sin (\omega t)+\Phi_{\text {bias }} \\
\theta(t)=\theta_{0} \cos (\omega t)
\end{gathered}
$$


where $\Phi_{0}$ is the amplitude of the roll motion, $\theta_{0}$ is the amplitude of the pitch motion, and $\Phi_{\text {bias }}$ is a mean roll bias, and $\Phi$ and $\theta$ are defined to be zero where the foil planform is parallel to the ground plane. The resulting nominal angle of attack, $\alpha$, of the foil relative to the stationary fluid is given by,

$$
\alpha(t)=-\arctan \left(\frac{\omega r_{0.7} \Phi(t)}{U}\right)+\theta(t)
$$

where $U$ is the carriage speed. By convention, $\alpha$ is calculated at a point that is $70 \%$ of the distance from the axis of rotation to the foil tip in the spanwise direction, i.e. at a point $r_{0.7}$ from the origin.

The relevant non-dimensional parameters for harmonically oscillating foils generating thrust in unobstructed flow are the Strouhal number, $S t=\frac{2 r_{0.7} \Phi_{0} f}{U}$, the maximum angle of attack, $\alpha_{\max }$, and the Reynolds number, $R e=\frac{U c}{\nu}$, where $\nu \approx 10^{-6} \mathrm{~m}^{2} s^{-1}$ is the kinematic viscosity of water [21]. For operation near the boundary, we define the final independent experimental parameter as $H^{*}=\frac{h}{c}$, where $d$ is the distance from the tank bottom to the pitch rotation axis in the ground effect test zone assuming $\Phi_{\text {bias }}=0$, following [18].

Reynolds number was held constant throughout; the foil was towed at $U=0.5 \mathrm{~m} / \mathrm{s}$ in all trials, resulting in $R e=57,000$.

With carriage speed, foil geometry, and roll amplitude all constant, the desired $S t$ was achieved by varying the oscillatory frequency, $f$.

$H^{*}$ was varied by adjusting $d$ the pitch axis at $0^{\circ}$ roll and the boundary in the 'ground effect' zone (see Figure 1.2.7a) through changes in the height of the mount point on the overhead carriage.

For harmonic rolling and pitching, it is evident from equation (1.3) that the evolution of $\alpha(t)$ is not sinusoidal. For a given $\Phi_{0}$ and $U$, an iterative procedure was used to determine the value of $\theta_{0}$ that produced the desired $\alpha_{\max }$.

Trials were conducted for $S t=\left[\begin{array}{llll}0.3 & 0.4 & 0.5 & 0.6\end{array}\right], \alpha_{\max }=\left[\begin{array}{llll}20^{\circ} & 25^{\circ} 30^{\circ} 35^{\circ} 40^{\circ}\end{array}\right]$, and

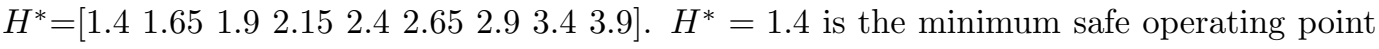
given the geometry of the foil apparatus. The parameter space was chosen to align with previous work in three dimensional flapping foils [21] and work with two dimensional foils by [18] to which we compare the data gathered in this experiment. At this height with a $12^{\circ}$ roll amplitude, the closest approach to the bottom by the foil tip is $1 \mathrm{~cm} . \Phi_{0}=12^{\circ}$ was maintained throughout all trials. 


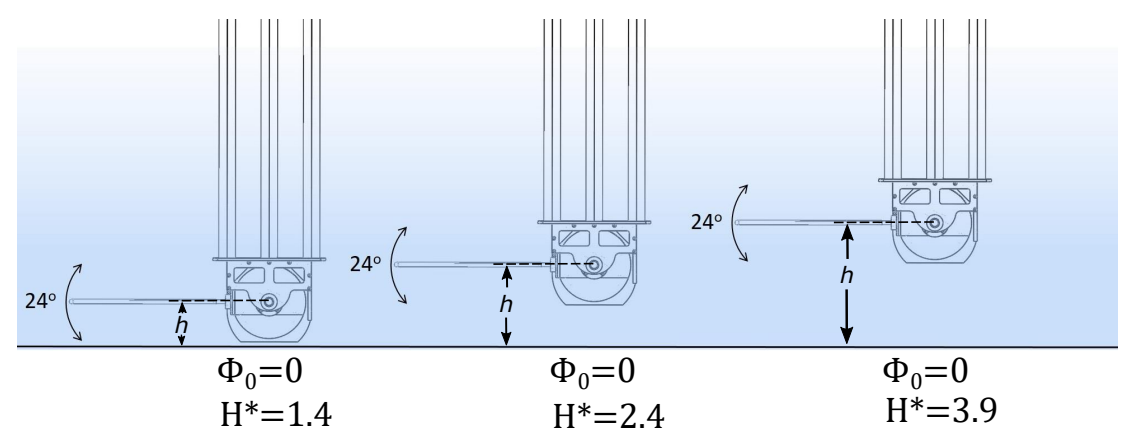

(a)

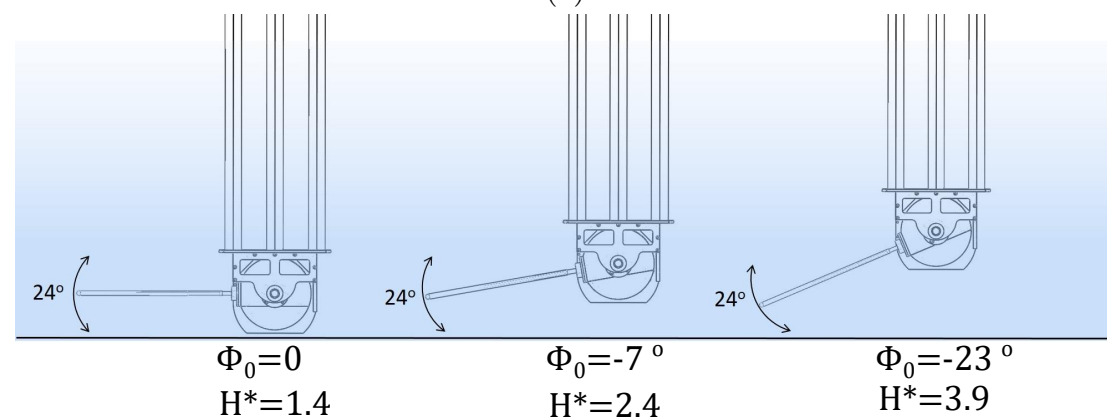

(b)

Figure 1.2.7: (a) The three dimensional foil with different values of $H^{*}$ with no roll bias (3-D case). (b) Test apparatus shown at multiple values of $H^{*}$ with a mean roll bias added to maintain a $1 \mathrm{~cm}$ tip clearance from the bottom. (3-D+ case)

When $\Phi_{\text {bias }}$ is held constant at zero, increasing $H^{*}$ increases the closest approach of the foil tip to the bottom. However, for each height above $H^{*}=1.4$, there is a non-zero $\Phi_{\text {bias }}$ such that the closest approach of the foil tip is returned to $1 \mathrm{~cm}$. For all values of (St, $\alpha_{0}$, $\left.H^{*}\right)$ tests were performed both with $\Phi_{\text {bias }}=0$ and with the appropriate $\Phi_{\text {bias }}$ to achieve $1 \mathrm{~cm}$ tip clearance. The maximum roll excursion of the foil is illustrated in the scale drawing in Figure 1.2.7b.

\subsubsection{Experimental Procedure}

Before each trial, the carriage is positioned against a hard stop at the deep end of the tank. The 6-axis dynamometer is tared to compensate for the in water weight of the foil before the flapping motion is started. The foil motion is started, and after three flapping cycles are completed, the carriage is accelerated up to $0.5 \mathrm{~m} / \mathrm{s}$ and travels the length of the tank, starting in freestream zone and ending in the ground effect test zone. Each run lasts approximately 40 seconds. After the carriage is returned to its starting position, the tank is allowed to settle for eight minutes before the next trial. 


\subsection{Results}

In this effort, we will focus on the mean lift and thrust produced by the oscillating foil, and the changes that occur when the foil moves from the freestream into proximity with the tank bottom. Results are reported in terms of non-dimensional lift and thrust coefficients, $C_{L}$ and $C_{T}$, respectively,

The instantaneous lift and thrust coefficients are calculated as follows,

$$
\begin{aligned}
C_{T} & =\frac{F_{x}}{0.5 \rho U^{2} S c} \\
C_{L} & =\frac{F_{z}}{0.5 \rho U^{2} S c}
\end{aligned}
$$

where $\rho$ is the density of water, $U$ is the carriage speed, and $S$ and $c$ are the foil span and chord lengths. Representative time series' of dimensional thrust and lift forces, along with the corresponding roll angle, are given for an example case where $S t=0.5, \alpha_{\max }=35^{\circ}$ in Figure 1.3.1.

$\bar{C}_{L}$ and $\overline{C_{T}}$ denote mean lift and thrust coefficients averaged over a complete cycle of motion, and are calculated as follows,

$$
\begin{aligned}
\bar{C}_{T} & =\frac{\int_{\tau_{i}}^{\tau_{i}+T} C_{T}(t) \mathrm{d} t}{T} \\
\bar{C}_{L} & =\frac{\int_{\tau_{i}}^{\tau_{i}+T} C_{L}(t) \mathrm{d} t}{T}
\end{aligned}
$$

where $T$ is the period of the motion and $\tau_{i}$ is the starting time for the $i^{\text {th }}$ cycle.

In each trial, the force data is divided into successive complete cycles, with each cycle identified using the starting and ending location of the foil within the tank. The individual cycles can then be labeled as occurring wholly within the freestream, wholly or in part within the transition zone, or wholly within the ground effect test zone. Cycles where the carriage is at rest or accelerating are discarded. 

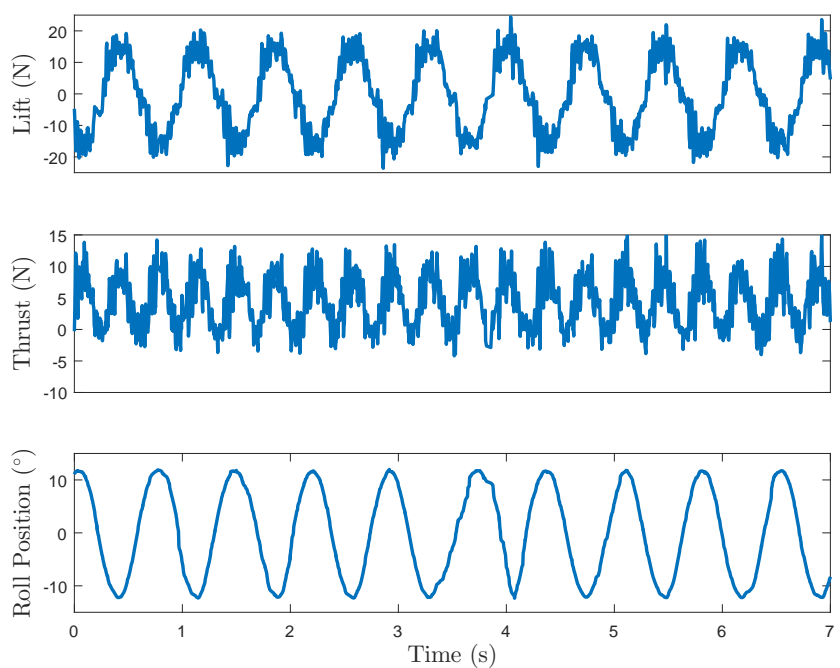

Figure 1.3.1: Lift force, thrust force, and roll angle measurement time series' while in the freestream zone, prior to phase averaging, for an example case withe $S t=0.5, \alpha_{\max }=35^{\circ}$.

\subsubsection{Evolution of $\overline{C_{L}}$ with transition into ground effect test zone.}

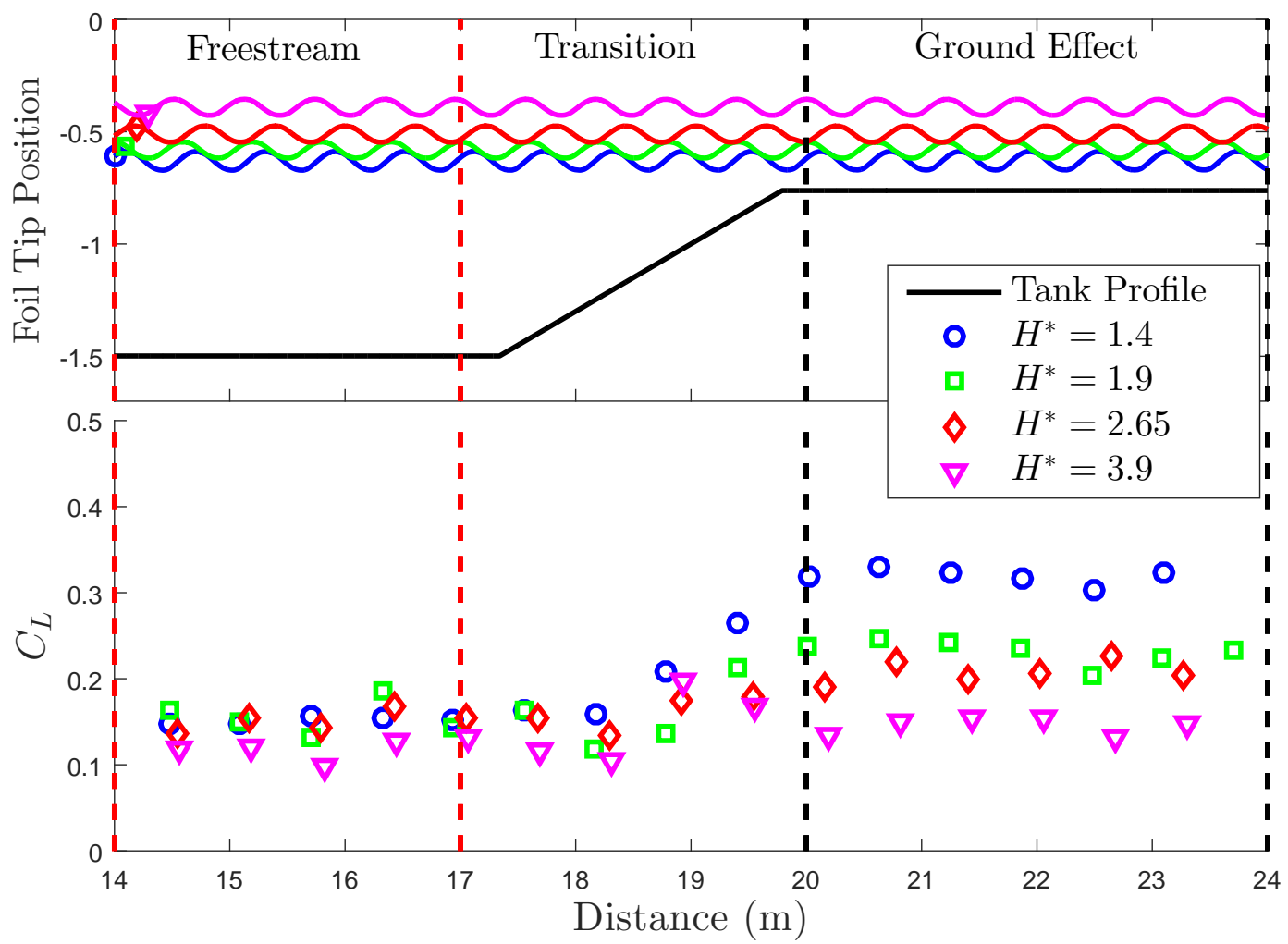

Figure 1.3.2: Tip position and lift coefficients of successive cycles as the foil moves down the tank for $\mathrm{St}=0.3, \alpha_{\max }=30^{\circ}$ and $H^{*}=[1.4,1.9,2.65,3.9]$ 
To illustrate the evolution of lift as the foil approaches and then operates near the bottom, $\bar{C}_{L}$ is plotted for each cycle as a function of carriage position for three cases ranging from $H^{*}=1.4$ to $H^{*}=3.9$ in Figure 1.3 .2 , along with the position of the foil tip relative to the bottom profile of the tank. The vertical lines show the boundaries of the three zones. The mean lift coefficient in the freestream zone for all cycles and all cases fall between 0.1 and 0.15 , indicating a small systemic bias in the alignment of the foil or apparatus. For the closest case, $H^{*}=1.4$ there is a consistent positive change in lift, indicating repulsion from the bottom, as the foil operates in the ground effect test zone. At $H^{*}=3.9$, no significant change is evident.

As $H^{*}$ decreases and the foil apparatus approaches the boundary, the flow beneath the apparatus will become constricted. We do not control for the fact that the drive system is being moved farther from the ground along with the foil and therefore we cannot directly separate the direct effects on the foils from these secondary effects due to the change in the interaction between the flow and drive system. The foil protrudes $5 \mathrm{~cm}$ from the leading edge of the drive mechanism minimizing turbulence from boundary layer effects but this is also a possible avenue for future investigation of near ground flapping foil propulsion. Any vehicle or drive system will constrict the flow beneath the body and should be expected to have similar vertical extent given similar foil geometry, hence the results should be broadly applicable to other three dimensional flapping foil systems.

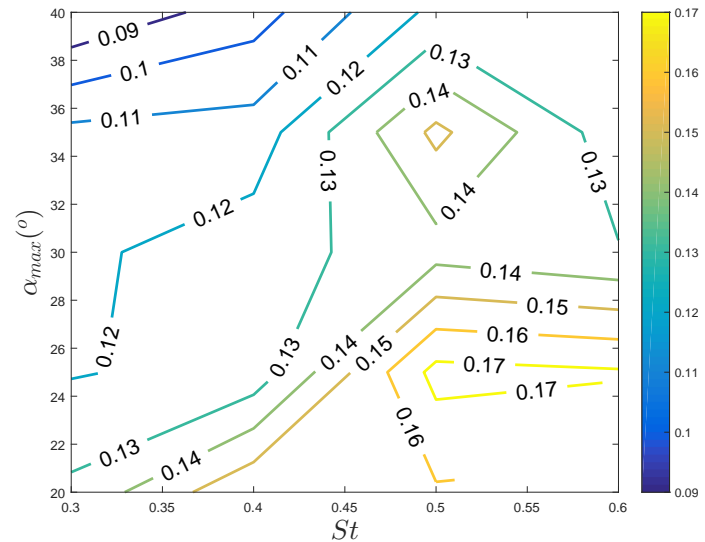

(a) Freestream Lift Coefficient, $\bar{C}_{L}$

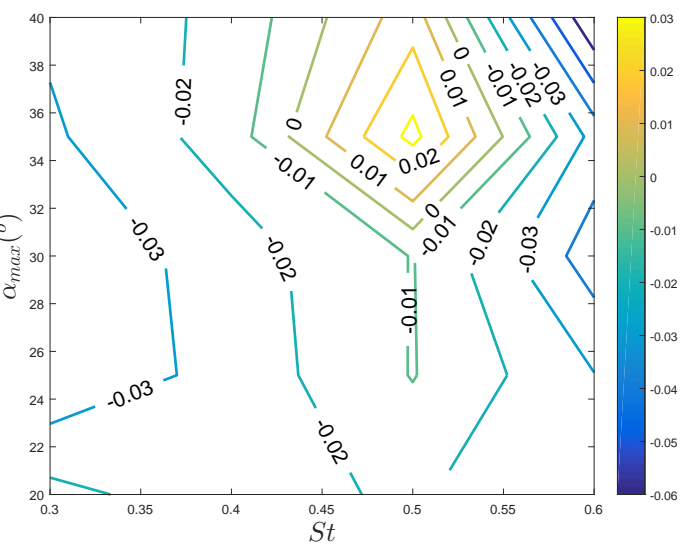

(b) Change in Freestream Lift Coefficient

Figure 1.3.3: (a)Mean lift coefficient, $\bar{C}_{L}$, while operating in the freestream for run corresponding to $H^{*}=3.9$ in the ground effect zone. (b) Difference in mean lift coefficient while operating in the freestream during trials of $H^{*}=1.4$ and $H^{*}=3.9$.

Figure 1.3.3a shows a positive mean lift coefficient in the freestream zone for all kine- 
matics tested produced by misalignment in the foil and drive mechanism; for a sense of the probable extent of the misalignment, we found that the magnitude of the lift is consistent with a non-zero pitch bias on the order of a degree. Figure 1.3.3b shows the change in the freestream lift coefficient from $H^{*}=1.4$ to $H^{*}=3.9$ showing that for all kinematics tested, the distance to the free surface of the tow tank has relatively small effect (ranging from -0.03 to 0.02 ) compared to the systemic bias due to misalignment or the change in lift due to ground effect $(>0.1)$. Comparison between freestream and near ground effect within a single experiment (i.e. a single traversal of the towing tank), rather than in two separate experiments, was used to ensure that any systemic biases due to misalignment of foil or drive system are included in the forces experience in both zones, and we can therefore reasonably assume that the gross effect of the misalignment is canceled. The current experiments do not allow us to assess the direct effect of the misalignment on the total magnitude of the ground effect, and this is an area of potential future efforts. For the purposes of discussion, we assume that this is a secondary effect that can be neglected.

\subsubsection{Variation in mean thrust}

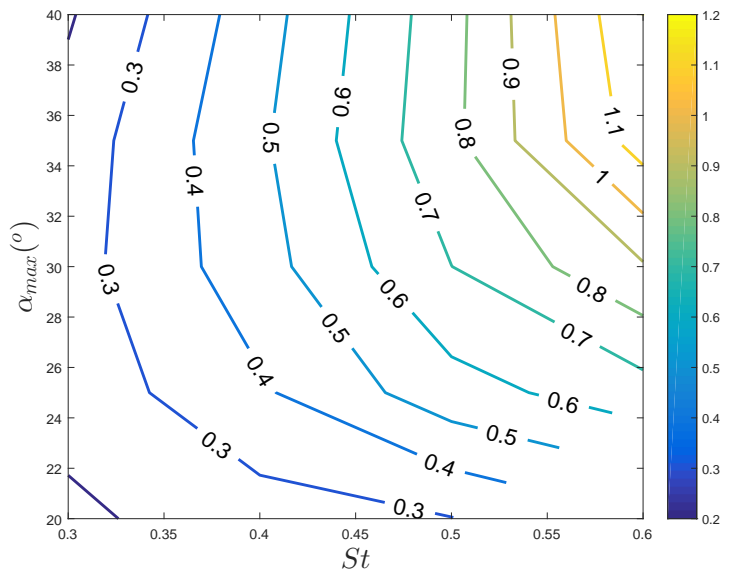

(a) Measured $\bar{C}_{T}$

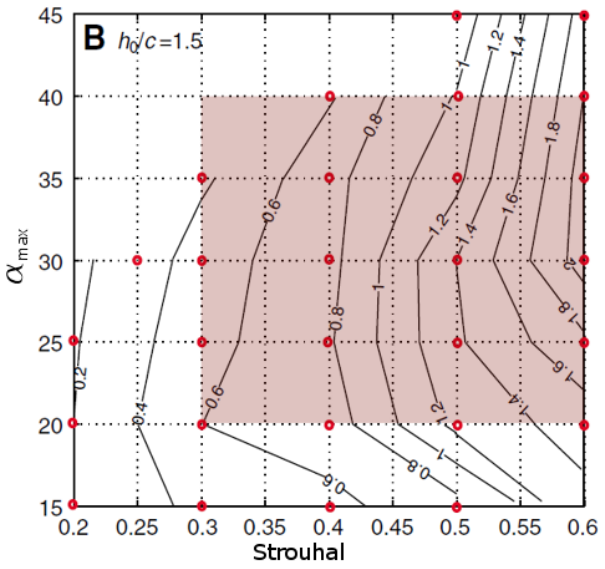

(b) Source: [21]

Figure 1.3.4: (a) Mean freestream thrust coefficient, $\overline{C_{T}}$, present work. (b) $\overline{C_{T}}$ as reported by [21] for a rolling and pitching foil operated with $\frac{h_{0.7}}{c}=1.5$. The present work covers a subset of the parameter space shown here, as highlighted in red.

Contours of mean thrust coefficient in the freestream zone are shown in Figure 1.3.4a. $\overline{C_{T}}$ increases with $S t$ for all $\alpha_{\max }$, consistent with previous research in both two dimensional [11] [18] and three dimensional foils [25] [20] [21]. Values of $\overline{C_{T}}$ reported by [21], for a 


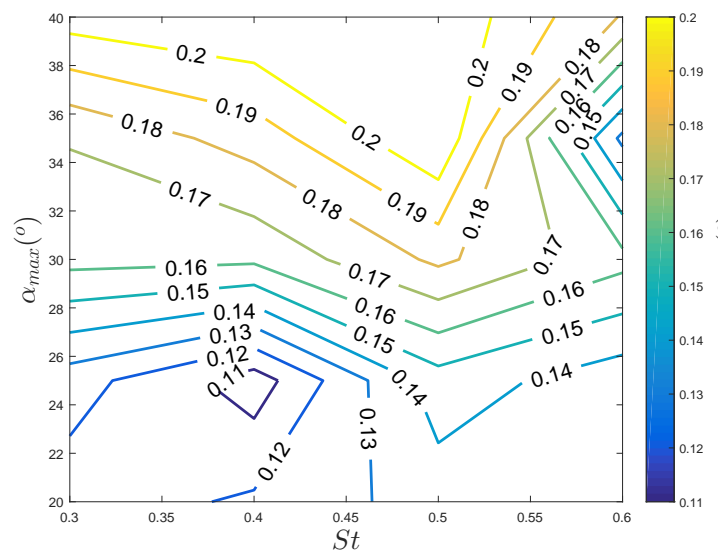

(a) Lift Coefficient Change $\Delta \bar{C}_{L}$

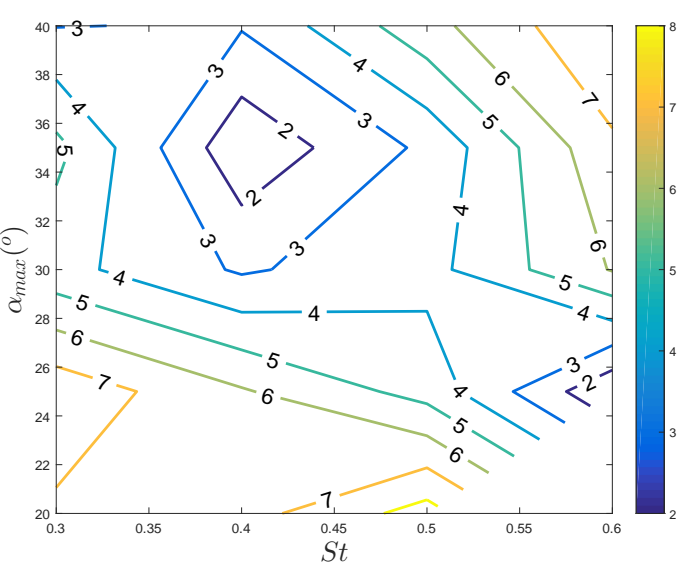

(b) Thrust Change, $\Delta \bar{C}_{T}[\%]$

Figure 1.3.5: Differences in the lift coefficient(a) and thrust coefficient(b), with the foil operating at the closest approach to the bottom $\left(H^{*}=1.4\right)$ vs. operation in the freestream $\left(H^{*} \approx 9\right)$.

rolling and pitching foil driven by a similar apparatus in a flow tunnel, are shown for direct comparison in Figure 1.3.4b. Testing across larger parameter set, [21] reports higher total thrust across the subset of parameters in the current work, with lower sensitivity to nominal angle of attack at low values of angle of attack. The difference in thrust coefficient may be caused by a number of differences in the geometry of those experiments, including the larger distance from axis of roll rotation to the foil base (caused by the use of an inline force sensor), the larger aspect ratio of the foil itself, and the use of a tapered, rather than a straight, trailing edge.

To illustrate the variation in the magnitude of ground effect as a function of the kinematic parameters, contours of the change in $\overline{C_{T}}$ and $\overline{C_{L}}$ between the freestream and the ground effect zone (denoted in the following as $\Delta \bar{C}_{T}$ and $\Delta \bar{C}_{L}$ ) at the closest approach to the bottom are shown in Figure 1.3.5. For $H^{*}=1.4$ case, a positive change in $\Delta \bar{C}_{L}$ was found for all kinematics tested, with the magnitude of the change ranging from 0.1 to 0.2 . The greatest repulsion from the bottom was found at the highest $\alpha_{\max }$ across all St. As shown in Figure 1.3.5b, $\Delta \bar{C}_{T}$ is always positive through all kinematics tested. Changes in thrust range in magnitude from 2 to 7 percent relative to the corresponding kinematics in the freestream. The greatest relative changes are found at high $S t$ with high $\alpha_{\max }$ and at low $\alpha_{\max }$ across low to moderate $S t$. Given that the magnitude of $\overline{C_{T}}$ at $\left(S t, \alpha_{\max }\right)=\left(0.6,40^{\circ}\right)$ is more than three times the magnitude at $\left(S t, \alpha_{\max }\right)=\left(0.5,20^{\circ}\right)$, the largest absolute increase in thrust occurs at high $S t$ and high $\alpha_{\max }$. 


\subsubsection{Change in lift and thrust in response to changes in proximity and foil orientation.}

Figure 1.3.7a shows the magnitude of $\Delta \bar{C}_{L}$ as a function of the proximity to the ground, $H^{*}$, for all values of $\alpha_{\max }$ at $S t=0.3$. Each curve represents a different $\alpha_{\max }$. Results with $\Phi_{\text {bias }}=0$ (referred to in the following as the 3-D case) are shown in Figure 1.3.7a. Results with $\Phi_{\text {bias }}$ set to bias the foil motion downwards as much as possible without contacting the bottom (referred to in the following as the $3-\mathrm{D}+$ case) are shown in Figure 1.3.7b.

The 3-D and 3-D + case show qualitatively similar trends in $\Delta \bar{C}_{L}$ with $H^{*} . \Delta \bar{C}_{L}$ is always positive (i.e. in no case is there a mean suction force towards the bottom), and always increasing as $H^{*}$ is decreased. For all $H^{*}$, the magnitude of $\Delta \bar{C}_{L}$ increases with increasing $\alpha_{\max }$. For the 3 -D + case, $\Delta \bar{C}_{L}$ is always greater than or equal to the 3 - $\mathrm{D}$ case, with the difference decreasing as the axis of the roll motion approaches the boundary and the kinematics converge. That is, biasing the foil downward towards the bottom increases the magnitude of the ground effect.

\subsubsection{Comparison to change in lift with two dimensional foil kinematics.}

One motivation for this investigation is to determine what qualitative and quantitative differences in observed ground effect occur between three dimensional rolling and pitching foils, and two dimensional heaving and pitching foils.

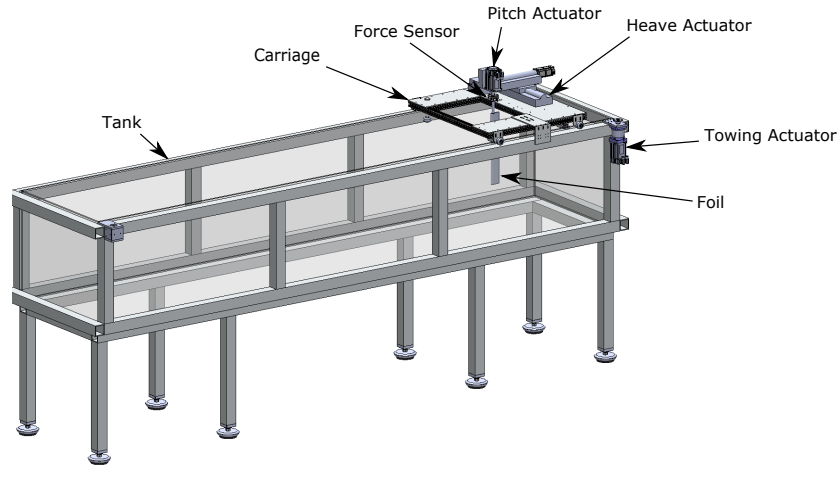

(a)

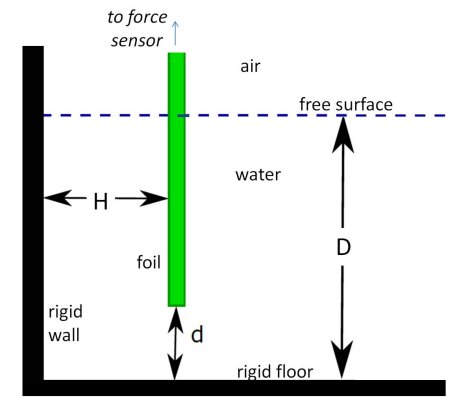

(b)

Figure 1.3.6: Experimental setup for previous work done with two dimensional foils [18]

To that end a comparison is made in Figure 1.3.7 between the results from the present effort, and experimental results produced using the experimental method detailed by [18], where a two dimensional heaving and pitching foil was tested in small tow tank. The 
apparatus is detailed in Figure 1.3.6a, taken from the previous work. Tests were performed with an aluminum foil with a constant NACA-0012 cross section, with chord length $0.07 \mathrm{~m}$ and submerged span of $0.37 \mathrm{~m}$, resulting in an effective aspect ratio of $5.3 . H^{*}$ was defined as $H^{*}=\frac{H}{c}$ for these experiments, where $H$ is the mean distance from the boundary to the foil as shown in Figure 1.3.6b, again taken from the previous work. Experiments were performed (a) with the tip of the foil less than $3 \%$ of the chord length from the bottom, and (b) with the tip of the foil $75 \%$ of the chord length from the bottom, i.e. with $d=2 \mathrm{~mm}$ and $d=52 \mathrm{~mm}$ in Figure 1.3.6b. The first configuration is assumed to result in nearly two dimensional fluid flow, while the second configuration allows spanwise flow around the tip. Both cases are 2-D combined heaving and pitching foils according to the classification in Section 1.1-1.3. In the following, we refer to the first configuration as the 2-D case, and the second configuration as the $2-\mathrm{D}+$ case, in order to distinguish them from one another.

Results used for comparison to the 2-D+ case in the present work are identical to those presented by [18]. A brief comparison between the 2-D and 2-D+ cases was included in the previous work, using 2-D results at a single value of $S t=0.35$. An expanded set of results for the 2-D case including multiple values of $S t$, again acquired using the same method, is used for comparison in the present work.

$\Delta \bar{C}_{L}$ for all $\mathrm{H}^{*}$ and $\mathrm{St}=0.3$ are shown in Figure $1.3 .7 \mathrm{c}$ and Figure $1.3 .7 \mathrm{~d}$, for the 2-D and 2-D+ case, respectively. In the 2-D case, a large suction force is present at low angles of attack, with $\Delta \bar{C}_{L}$ becoming increasingly negative as the foil approaches the solid boundary. $\Delta \bar{C}_{L}>0.05$ is only achieved at the very highest $\alpha_{\max }$ with the very closest approach, $H^{*}=1.33$. In contrast, the rolling and pitching foil always shows an increasingly positive repulsion force as the boundary is approached, regardless of $\alpha_{\max }$.

When flow around the tip is allowed, as in the 2-D+ case, we no longer see the large suction forces present in 2-D case at low $\alpha_{\max } . \Delta \bar{C}_{L}$ at the closest approach increases monotonically with $\alpha_{\max }$, from approximately zero at $\alpha_{\max }=20^{\circ}$ to nearly 0.2 at $\alpha_{\max }=$ $40^{\circ}$. At $\alpha_{\max }=40^{\circ}$ the change in lift is similar for the 2-D+ and the 3-D case, however in the 3-D case the change is less sensitive to $\alpha_{\max }$. As in the 3-D case, the effect of the boundary is negligible for $H^{*}>3$ for both the 2-D and 2-D+ cases. 


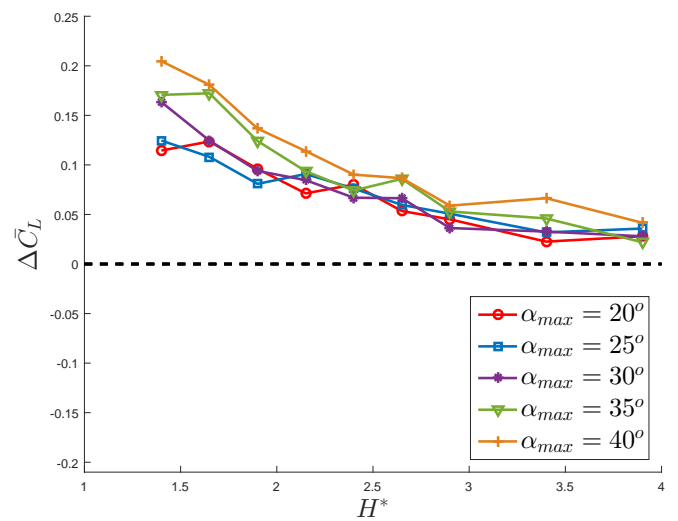

(a) 3 -D case $(\mathrm{St}=0.3)$

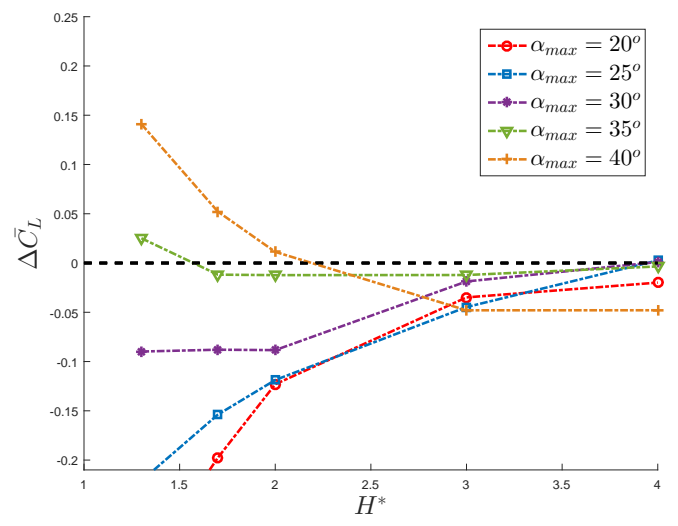

(c) $2-\mathrm{D}$ case $(\mathrm{St}=0.3)$

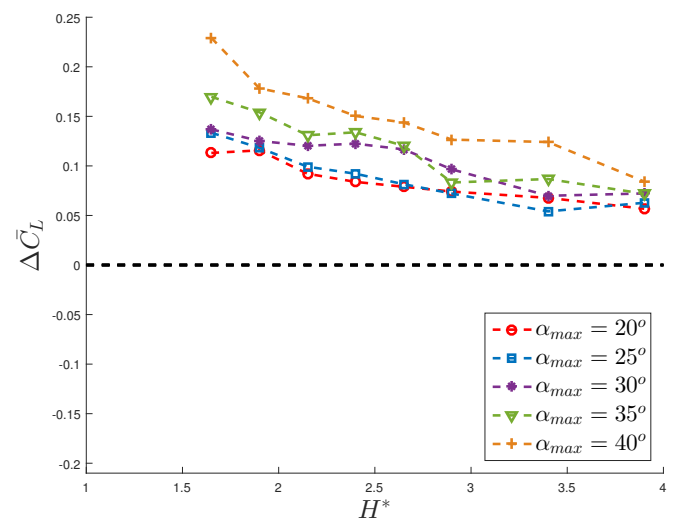

(b) $3 \mathrm{D}+$ case $(\mathrm{St}=0.3)$

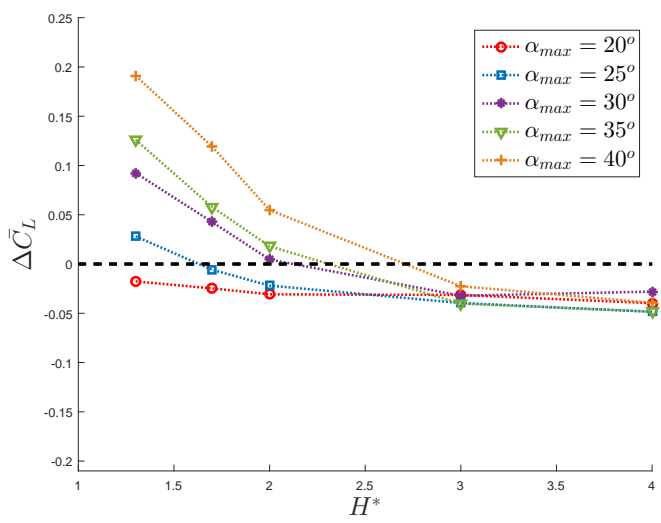

(d) 2 -D+ case $(\mathrm{St}=0.3)$

Figure 1.3.7: $\Delta \bar{C}_{L}$ due to ground effect in 3D: rolling and pitching foils (a), and 3D+: rolling and pitching foils with a mean downward roll bias (b). Results from [18] in 2D: heaving and pitching with no spanwise flow (c), and 2D+: heaving and pitching with spanwise flow (d), 


\subsubsection{Cross platform comparison across a range of kinematic parameters.}

A comparison between two-dimensional and three-dimensional foils, Figure 1.3.8, shows that difference in $\Delta \bar{C}_{L}$ between the two depends on $S t$ and $\alpha_{\max }$. The two are qualitatively similar at low $S t$ and high $\alpha_{\max }$ with a minimum difference in mean coefficient of lift of 0.06 but diverge as $S t$ increases and $\alpha_{\max }$ decreases with a maximum difference of 0.85 during the closest approach $\left(H^{*}=1.4\right)$.
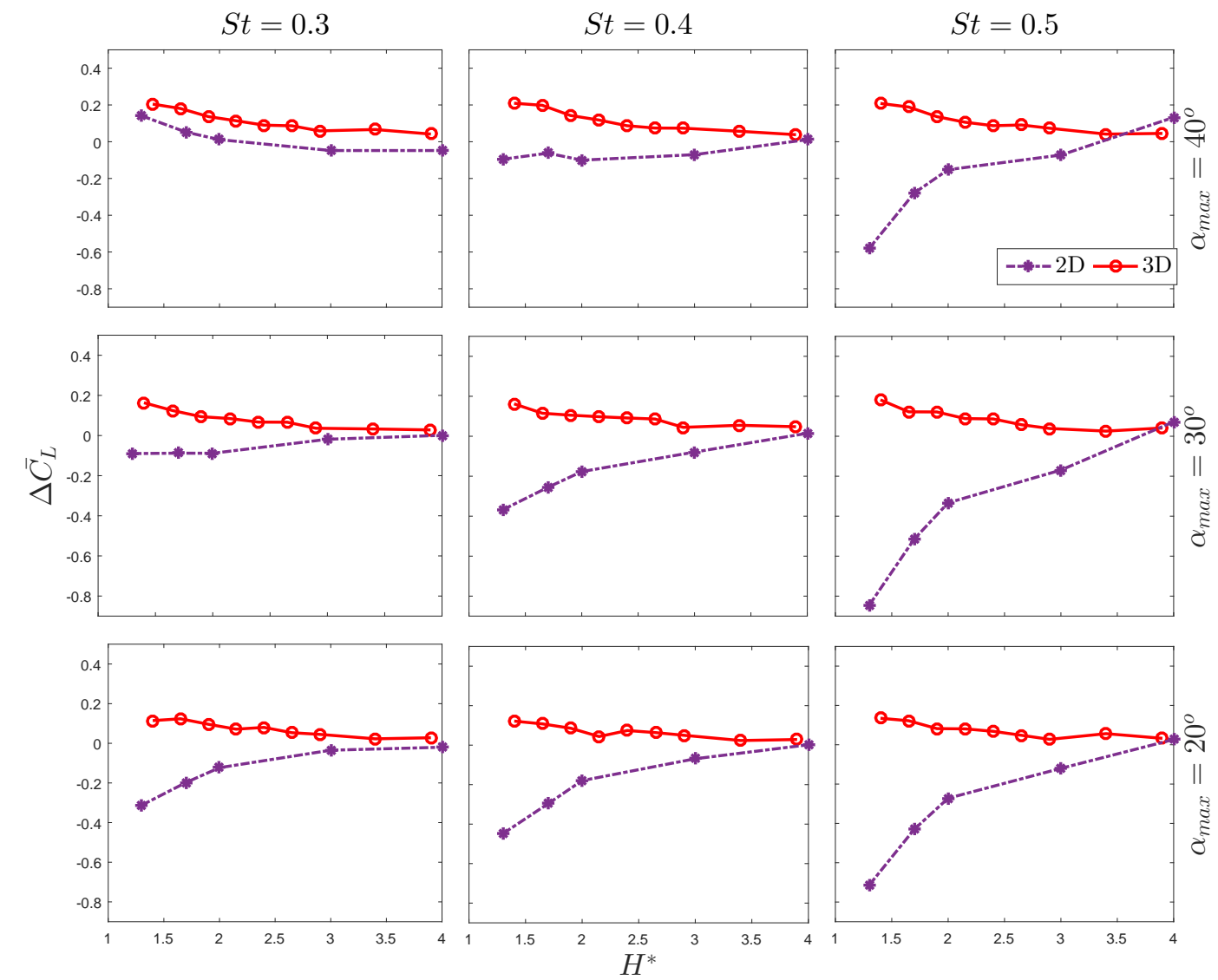

Figure 1.3.8: Comparison of 2-D and 3-D cases for $S t=[0.3,0.4,0.5]$ and $\alpha_{\max }=\left[20^{\circ}, 30^{\circ}, 40^{\circ}\right]$. Results from [18], including previously unpublished experiments, are compared to the present work. We define heaving and pitching without flow around the tip as the '2-D' case opposed to rolling and pitching with flow around the tip as the

' 3 -D' case.

To better visualize the differences in $\Delta \bar{C}_{l}$ as a function of $H^{*}$ directly compared across all four foil configurations (2-D, 2-D+, 3-D, 3-D+), the scale was adjusted in Figure 1.3.9, which led to some of the 2-D data points to lay outside the visible window. In Figure 1.3.9 the results for nine underlying cases, representing the entire range of kinematic parameters 
tested, are shown, with $S t=[0.3,0.4,0.5]$ and $\alpha_{\max }=\left[20^{\circ}, 30^{\circ}, 40^{\circ}\right]$.
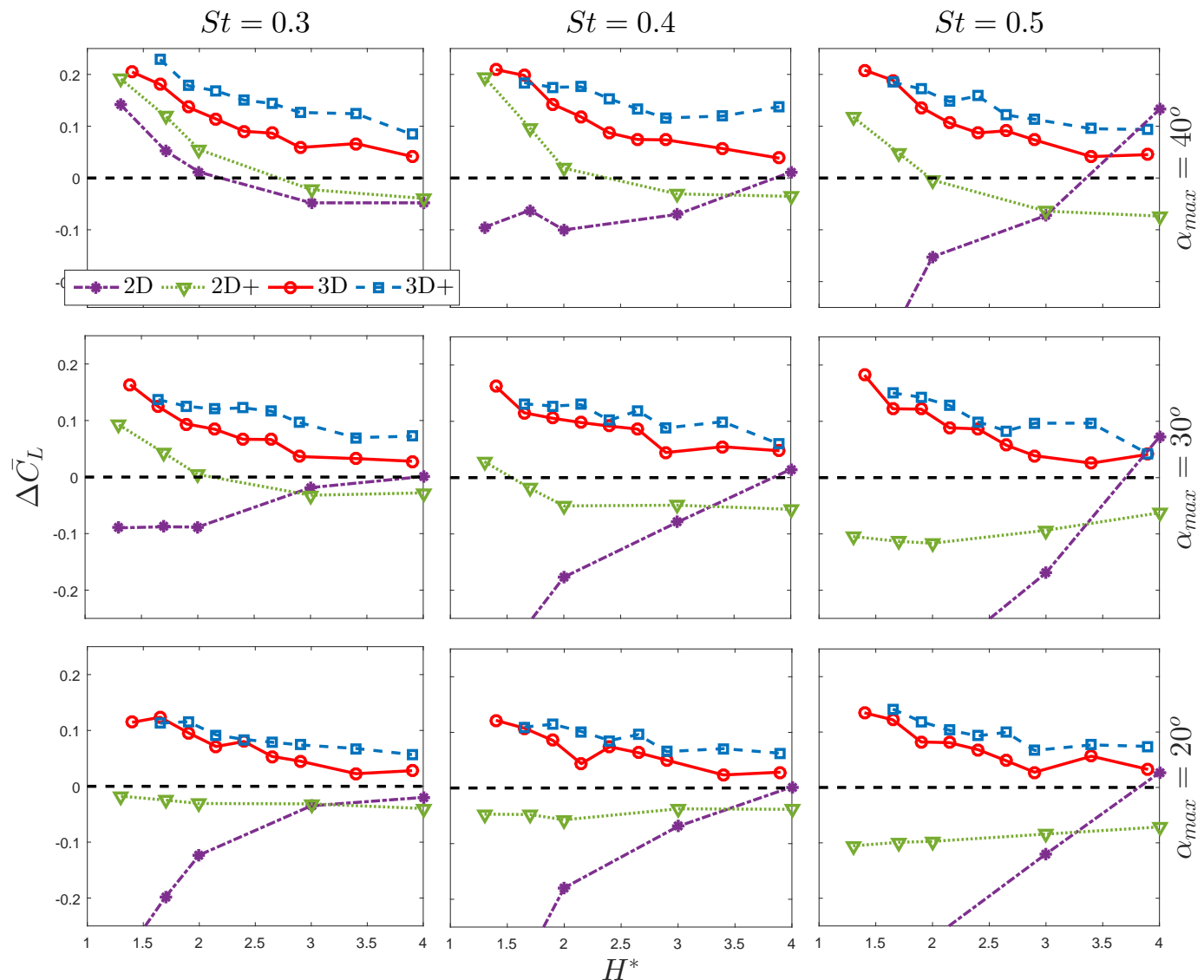

Figure 1.3.9: Comparison of 2-D, 2-D+, 3-D, 3-D + cases for $S t=[0.3,0.4,0.5]$ and $\alpha_{\max }=\left[20^{\circ}, 30^{\circ}, 40^{\circ}\right]$. Results from [18], including previously unpublished experiments, are compared to the present work. We define heaving and pitching without flow around the tip as the '2-D' case, heaving and pitching with flow around the tip as the '2-D+' case, rolling and pitching with flow around the tip as the ' 3 -D' case, and rolling and pitching with flow around the tip and a downward roll bias such that the minimum safe tip height is reached as the ' $3-\mathrm{D}+$ ' case. Extreme values of the '2-D' case were excluded in order to focus on differences across all platforms in greater detail.

Across all underlying foil kinematics, the $3 \mathrm{D}$ and $3 \mathrm{D}+$ cases see a positive $\Delta \bar{C}_{L}$ as the foil approaches the solid boundary. The magnitude of $\Delta \bar{C}_{L}$ increases with increasing $\alpha_{\max }$ for all $\alpha_{\max }$, and $\Delta \bar{C}_{L}>0.05$ within $H^{*}<3$ for all cases.

Biasing the foil downward towards the bottom increases the magnitude of the ground effect across the entire range of kinematics tested, i.e. $\Delta \bar{C}_{L}$ for the $3-\mathrm{D}+$ case is always greater than or equal to the 3-D case, with the difference decreasing as the kinematics of the two cases converge. In the 3 -D+ case, angling the foil downwards towards the bottom results in $\Delta \bar{C}_{L}>0.05$ at the largest distance tested, $H^{*}=3.9$ with the exception of 
$\left(S t, \alpha_{\max }\right)=\left([0.4,0.5], 30^{\circ}\right) . \Delta \bar{C}_{L}>0.05$ for $H^{*} \leq 3.4$ in all cases.

In contrast, for the purely $2-\mathrm{D}$ cases $\Delta \bar{C}_{L}$ is negative at the closest approach for all cases except for $\left(S t, \alpha_{\max }\right)=\left(0.3,40^{\circ}\right)$, indicating a suction force drawing the foil towards the boundary. As $\alpha_{0}$ decreases and/or $S t$ increases, the $\Delta \bar{C}_{L}$ becomes increasingly negative.

In the $2-\mathrm{D}+$ case, $\Delta \bar{C}_{L}$ is positive for $\alpha_{\max } \geq 30$, with the exception of the case where $\left(S t, \alpha_{\max }\right)=\left(0.5,30^{\circ}\right)$. For all other kinematics, $C_{L}$ is less sensitive to $H^{*}$ in the 2 -D+ case, compared to the other cases.

\subsection{Discussion}

The presence of a nearby solid boundary influences the hydrodynamic forcing on an oscillating foil used for propulsion. We highlight three significant conclusions that can be derived from the experimental results presented above:

1. Foils used to generate high thrust with rolling and pitching oscillations experience a 'ground effect' which repels them from solid boundaries.

2. Ground effect can be induced at greater distances by biasing the tip of the foil toward the boundary.

3. The ground effect experienced by a high-aspect ratio rolling and pitching foil is a fully three-dimensional phenomenon, which cannot be accurately predicted when twodimensional flow and/or two-dimensional kinematics are enforced.

\subsubsection{Foils used to generate high thrust with rolling and pitching oscillations experience a 'ground effect' which repels them from solid boundaries.}

The results presented in Figure 1.3.9 make it clear that for the entire range of kinematics investigated, the presence of the boundary repels the foil from the boundary. The non-zero mean lift indicates asymmetry in the fluid wake resulting from boundary effects, as the foil kinematics themselves are symmetric.

In steady flow, absent obstructions or boundaries, symmetric foil oscillations with zero mean pitch angle produce zero mean lift over each complete cycle of motion [21]. The magnitude of the opposing instantaneous lift peaks is typically several times the magnitude of the mean thrust [20]. Given the presence of these large instantaneous lift forces, it is thus not unexpected that large mean lift forces can be produced through asymmetries in the kinematics. These large lift forces have been illustrated in bench top experiments [11] [13] 
[25] and in practical application on a vehicle [23], where a non-zero mean pitch angle (pitch

'bias') is used to vector total force and control the trajectory of an underwater vehicle.

Absent ground effect, a free-swimming vehicle (or animal) seeking to avoid a boundary would need to actively sense that boundary and alter foil kinematics to vector the total force away from it. However, the ubiquity of the ground effect in high thrust kinematics suggests that the ground effect can provide a passive obstacle avoidance capability. With proper trajectory design, the closed loop sense/react control problem could be alleviated by taking advantage of the naturally occurring cushion created by the ground effect.

\subsubsection{Ground effect can be induced at greater distances by biasing the tip of the foil toward the boundary.}

The magnitude of the repulsion force created by the ground effect is strongly dependent on the distance between the foil and the boundary. If the foil is oscillated straight out from the side of a swimmer, as in Figure 1.2.7a, any passive obstacle avoidance or sensing is then entirely a function of the swimmer height above bottom. To experience ground effect in this case, the swimmer body would necessarily be very close to the boundary, potentially only a fraction of the foil chord length.

In practice, it may be desirable for an underwater vehicle to maintain a greater stand off distance from a boundary for safe operation. The results indicate that operating with the tip of the foil biased in the direction of the boundary, as shown in Figure 1.2.7b is an effective way of increasing the distance at which the passive cushioning effect of the ground effect is experienced. While this strategy does not increase the magnitude of the ground effect, reducing the sensitivity to ground proximity has a significant practical implication.

\subsubsection{The ground effect experienced by a high-aspect ratio rolling and pitching foil is a fully three-dimensional phenomenon, which cannot be accurately predicted when two-dimensional flow and/or two-dimensional kinematics are enforced.}

Previous experimental and numerical studies of flapping foils have enforced artificial limitations on the degrees of freedom of the foil motion and the fluid flow. However, the results of the present work show that there are significant qualitative differences between the forces produced by two dimensional and three dimensional foil and flow geometries. Based on this observation, we believe that naively extrapolating from the two dimensional to the three dimensional using empirical coefficients based on aspect ratio, such as those presented 
for stationary foils in [26], is likely to fail.

One rationale for investigation of two dimensional foil kinematics and flow (i.e. infinite aspect ratio foils) is to inform strip theory estimates of total force on finite aspect ratio foils. This approach assumes that an effective 2D heave and pitch trajectory can be calculated for each section as a function of the spanwise location and the rotary kinematics, and that resulting forces based on comparable 2-D experiments can be integrated along the length of the foil. The results presented in Figure 1.3.8 suggest that this section by section approach is also likely to fail. The key problem with this approach is that for all effective trajectories other than those with low St number and high angle of attack (i.e. in the top left plot of Figure 1.3.8), the 2-D foil experiments predict a suction force where the 3-D foil experiments uniformly show a repulsive force. As an illustrative example, for $S t=0.4$ and $\alpha_{\max }=30^{\circ}$, the effective Strouhal number and $\alpha_{\max }$ will both be higher towards the tip of the foil, and they will both be lower towards the root of the foil. From Figure 1.3.8 it is evident that every nominal 2-D section along the span of the foil will produce a suction force, yet the foil as a whole produces a repulsion force!

For self-propelled underwater vehicles using variations on median paired pectoral fin propulsors [23] [27] [28] both the foil motion and the fluid flow are necessarily three dimensional. For underwater vehicles propelled using variations on the caudal fin [29] [30], the foil motion more closely approximates heaving and pitching with spanwise flow allowed around both tips, as in the 2-D+ case. Ground effect for vehicles that propel themselves with pitch only motions [31] [32] [33] are more appropriately modeled using results for very low aspect ratio foils with spanwise flow, e.g. from [7]

Studies of steady wing in ground effect studies may provide insight in to the differences in the force production of two and three dimensional flapping foils. [34] splits fixed wing ground effect into two phenomena one being span dominated and pertaining to the interaction between ground and the tip vortices and a second being chord dominated 'ram effect' that deals with high pressure build up under the wing. In the former, the tip vortices are compressed by the presence of the solid boundary increasing the effective span and also decreasing the strength or amount of downwash created by the tip vortices, which in turn decreases the energy lost as drag. [35] also notes that the decreased downwash changes the streamlines in the flow, increasing the effective angle of attack of the foil increasing lift. As the distance between the wing and the ground decreases ram effect increases as the air 
speed beneath the wing begins to stagnate increasing the pressure under the wing. However, with convex foils, at low angles of incidence, lift can actually be decreased as a venturi nozzle begins to form between the foil and the ground, creating suction between the two. It should also be noted that the chord dominated ground effect is a two-dimensional flow effect, whereas the decreased downwash of the tip vortices is a three-dimensional effect.

These phenomena can help explain the difference in results between the 2-D, 2-D+ and $3-\mathrm{D}$ cases. In the 2-D case there should be no tip vortex present, eliminating any lift enhancement or drag reduction due to their compression. The 2-D case also sees the highest amounts of suction at lower maximum angles of attack which corresponds with the conditions for the formation of a Venturi nozzle. In the 2-D+ case, there should be tip vortex effects which would counteract the suction dominated forces and explain the increase in $\Delta \bar{C}_{L}$ from the $2-\mathrm{D}$ to the $2-\mathrm{D}+$ case across the entire test range. The $3-\mathrm{D}$ case is likely dominated by tip vortex boundary interactions since the roll motion of the foil would cause large amounts of spanwise flow, likely preventing the formation of a Venturi nozzle and therefore eliminating any suction effects.

The force data presented may provide first order validation data for fully three dimensional numerical flow simulations. Experiments with either qualitative flow visualization

(e.g. dye release) or quantitative flow visualization (e.g. particle image velocimetry, or PIV) are an important avenue for future research efforts, and have the potential to provide more insight in to the underlying physical mechanisms involved as well as more fine grained validation of numerical simulations.

\subsection{Conclusion}

The present work demonstrates that it is critical to investigate ground effect using fully three dimensional geometry. Swimming animals and biologically inspired underwater vehicles use a wide variety of different oscillating foil mechanisms to propel themselves. In the face of this variety, it is natural to seek fundamental knowledge through experimental and numerical investigations which simplify the underlying hydrodynamic mechanisms. As a result, there is a large body of work into two dimensional foil and flow geometries, including numerous studies that attempt to elucidate ground effect with two dimensional numerical simulations. However, extrapolating two dimensional results through techniques such as strip theory, or empirical correction factors for aspect ratio, will fail to capture the qualitative 
and quantitative differences between the two dimensional and three dimensional cases, which are strongly dependent on nominal foil kinematics.

Productive directions for future efforts include flow visualization or numerical simulations to illuminate the underlying hydrodynamic mechanisms at work in ground effect; variation of foil geometry, particularly aspect ratio; variation of foil kinematics along with the inclusion of in-line motion similar to work by [13]; and introduction of soft or compliant elements in the foil structure to more closely imitate biological templates. These investigations can provide fundamental performance data to directly inform the design of vehicle propulsion, sensing, and control systems. With sufficient precision and calibration, force feedback could be used to not only detect boundaries but estimate distances to the boundary as well. Computational fluid dynamics (CFD) techniques are only beginning to approach the practical capability to model unsteady, 3-D fluid-structure interaction at Reynolds numbers approaching those of practical unmanned underwater vehicles. We hope that this and future experimental studies will eventually provide a rich data set for validation of these CFD tools as their sophistication and capabilities improve.

\section{List of References}

[1] M. M. Koochesfahani, "Vortical patterns in the wake of an oscillating airfoil," American Institute of Aeronautics and Astronautics, vol. 27, no. 9, pp. 1200-1205, Sept 1989.

[2] K. D. Jones, C. M. Dohring, and M. F. Platzer, "Experimental and Computational Investigation of the Knoller-Betz Effect," AIAA Journal, vol. 36, no. 7, pp. 1240-1246, July 1998.

[3] L. Dai, G. He, and X. Zhang, "Self-propulsion of a Flexible Plunging Foil Near a Solid Wall," Procedia Engineering, vol. 126, pp. 431-435, 2015.

[4] L. Dai, G. He, and X. Zhang, "Self-propelled swimming of a flexible plunging foil near a solid wall," Bioinspiration E Biomimetics, vol. 11, no. 4, p. 046005, July 2016.

[5] J. Ryu, S. G. Park, B. Kim, and H. J. Sung, "Flapping dynamics of a flexible propulsor near ground," Acta Mechanica Sinica, vol. 32, no. 6, pp. 991-1000, Dec. 2016.

[6] C. Zhang, H. Huang, and X.-Y. Lu, "Free locomotion of a flexible plate near the ground," Physics of Fluids, vol. 29, no. 4, p. 041903, Apr. 2017.

[7] D. B. Quinn and A. J. Smits, "Rectangular panels undergoing pitch oscillation in ground effect," in The 1000 Island Fluids Meeting, 2012.

[8] D. B. Quinn, K. W. Moored, P. A. Dewey, and A. J. Smits, "Unsteady propulsion near a solid boundary," Journal of Fluid Mechanics, vol. 742, pp. 152-170, Mar. 2014.

[9] R. Fernandez-Prats, V. Raspa, B. Thiria, F. Huera-Huarte, and R. Godoy-Diana, "Large-amplitude undulatory swimming near a wall," Bioinspiration $\&$ biomimetics, vol. 10, no. 1, p. 016003, 2015. 
[10] J. M. Anderson, K. Streitlien, D. S. Barrett, and M. S. Triantafyllou, "Oscillating foils of high propulsive efficiency," Journal of Fluid Mechanics, vol. 360, pp. 41-72, Apr. 1998.

[11] D. A. Read, "Oscillating foils for propulsion and maneuvering of ships and underwater vehicles," Master's thesis, Massachusetts Institute of Technology, 2001.

[12] P. Prempraneerach, F. S. Hover, and M. S. Triantafyllou, "The effect of chordwise flexibility on the thrust and efficiency of a flapping foil," Proceedings Unmanned, Untethered Submersible Technology, 2003.

[13] J. S. Izraelevitz and M. S. Triantafyllou, "Adding in-line motion and model-based optimization offers exceptional force control authority in flapping foils," Journal of Fluid Mechanics, vol. 742, pp. 5-34, Mar. 2014.

[14] S. C. Licht, M. S. Wibawa, F. S. Hover, and M. S. Triantafyllou, "In-line motion causes high thrust and efficiency in flapping foils that use power downstroke," The Journal of experimental biology, vol. 213, no. 1, pp. 63-71, 2010.

[15] E. Blevins and G. V. Lauder, "Swimming near the substrate: a simple robotic model of stingray locomotion," Bioinspiration \& Biomimetics, vol. 8, no. 1, p. 016005, Mar. 2013.

[16] D. B. Quinn, G. V. Lauder, and A. J. Smits, "Flexible propulsors in ground effect," Bioinspiration \& Biomimetics, vol. 9, no. 3, p. 036008, Sept. 2014.

[17] J. Wu, Y. L. Qiu, C. Shu, and N. Zhao, "Pitching-motion-activated flapping foil near solid walls for power extraction: A numerical investigation," Physics of Fluids, vol. 26, no. 8, p. 083601, Aug. 2014.

[18] A. Mivehchi, J. Dahl, and S. Licht, "Heaving and pitching oscillating foil propulsion in ground effect," Journal of Fluids and Structures, vol. 63, pp. 174-187, May 2016.

[19] S. Licht, V. Polidoro, M. Flores, F. Hover, and M. Triantafyllou, "Design and Projected Performance of a Flapping Foil AUV," IEEE Journal of Oceanic Engineering, vol. 29, no. 3, pp. 786-794, July 2004.

[20] V. Polidoro, "Flapping foil propulsion for cruising and hovering autonomous underwater vehicles," Master's thesis, Massachusetts Institute of Technology, 2003.

[21] A. H. Techet, "Propulsive performance of biologically inspired flapping foils at high Reynolds numbers," Journal of Experimental Biology, vol. 211, no. 2, pp. 274-279, Jan. 2008.

[22] S. L. Rauworth, "Development of an underwater flapping foil tow-test system," Master's thesis, University of Rhode Island, 2014.

[23] S. C. Licht, "Biomimetic oscillating foil propulsion to enhance underwater vehicle agility and maneuverability," Ph.D. dissertation, Massachusetts Institute of Technology and Woods Hole Oceanographic Institution, 2008.

[24] D. Elles, "Analysis of Force Production by a Biologically Inspired Underwater Flapping Foil Near Solid Boundaries in Three Dimensional Flow," 2016.

[25] M. D. Flores, "Flapping motion of a three-dimensional foil for propulsion and maneuvering of underwater vehicles," Ph.D. dissertation, Massachusetts Institute of Technology, 2003. 
[26] S. F. Hoerner and H. V. Borst, "Fluid-dynamic lift: practical information on aerodynamic and hydrodynamic lift," 1985.

[27] C. Siegenthaler, C. Pradalier, F. Gnther, G. Hitz, and R. Siegwart, "System integration and fin trajectory Design for a robotic sea-turtle," in 2013 IEEE/RSJ International Conference on Intelligent Robots and Systems, Nov. 2013, pp. 3790-3795.

[28] D. Beal, H. Leinhos, A. Fredette, and R. Berube, "Unified Scaling for Flapping Fins," IEEE Journal of Oceanic Engineering, vol. 38, no. 1, pp. 1-11, 2013.

[29] J. M. Anderson and N. K. Chhabra, "Maneuvering and Stability Performance of a Robotic Tuna," Integrative and Comparative Biology, vol. 42, no. 1, pp. 118-126, Feb. 2002 .

[30] M. Rufo and M. Smithers, "Ghostswimmer auv: Applying biomimetics to underwater robotics for achievement of tactical relevance," Marine Technology Society Journal, vol. 45 , no. $4,2011$.

[31] T. Salumäe, A. Chemori, and M. Kruusmaa, "Motion control architecture of a 4-fin u-cat auv using dof prioritization," in Intelligent Robots and Systems (IROS), 2016 IEEE/RSJ International Conference on. IEEE, 2016, pp. 1321-1327.

[32] J. H. Long Jr, J. Schumacher, N. Livingston, and M. Kemp, "Four flippers or two? tetrapodal swimming with an aquatic robot," Bioinspiration 85 Biomimetics, vol. 1, no. 1 , p. $20,2006$.

[33] C. Georgiades, M. Nahon, and M. Buehler, "Simulation of an underwater hexapod robot," Ocean Engineering, vol. 36, no. 1, pp. 39-47, 2009.

[34] T. Abramowski, "Numerical investigation of airfoil in ground proximity," Journal of Theoretical and Applied Mechanics, vol. 45, pp. 425-436, 2007.

[35] M. Ahmed and S. Sharma, "An investigation on the aerodynamics of a symmetrical airfoil in ground effect," Experimental Thermal and Fluid Science, vol. 29, no. 6, pp. 633-647, July 2005. 


\section{MANUSCRIPT 2}

\section{Free surface boundary effects on rolling and pitching oscillating foil propulsion}

This paper expands the research into boundary effects on force production of a rolling and pitching oscillating foil. An oscillating foil apparatus was towed in a fresh water tank operating over a range of kinematics with Strouhal numbers, St, from 0.3 to 0.5 and maximum angles of attack, $\alpha_{\max }$, from $20^{\circ}$ to $40^{\circ}$ at varying distances from the free surface, $H^{*}=1.75$ to 5.68 . Hydrodynamic forces were recorded and analyzed. Changes in forces as the foil approaches the free surface of the tow tank show the presence of a repulsion force similar to that experienced by an oscillating foil in ground effect. Lift coefficients were affected my magnitudes reaching 0.19 with values $>.05$ across all tested kinematics. The surface effect develops similarly to ground effect as an oscillating foil approaches the boundary. This suggests that similar hydrodynamic mechanisms are at work in both cases. Changes seen in mean thrust coefficients due to the free surface are found to be negative where as ground effect sees positive. The negative change can be explained by energy lost due to wave generation as the foil operates near the surface.

\subsection{Introduction and Background}

Biomimetic propulsion systems have long been an area of interest in underwater vehicles. With the development of new systems come new difficulties designing control systems to harness these novel propulsors. A step toward efficient and robust control of a vehicle is understanding the forces it undergoes while in operation. Oscillating foils are one novel propulsion method that has evolved from these studies. The goal of oscillating foils is to take inspiration from sea animals such as a sea turtle in order to inspire novel propulsion methods for underwater vehicles.

\subsubsection{Oscillating Foils}

In our work, we distinguish between different oscillating foil configurations by referring to the degrees of freedom in the motion of the foil. Three common configurations are one dimensional (pitching or heaving motions only), two dimensional (coupled heaving and pitching motions), and three dimensional (combined rolling and pitching motions). This paper focuses on three dimensional (3-D) rolling and pitching oscillating foils and is the first 
experimental study of 3-D high aspect ratio thrust producing foils near a free surface. One and two dimensional kinematics have been studied both numerically and experimentally.

[1] found experimentally that the structure of a wake behind a pitching only 1-D oscillating foil can be modified significantly by changing the amplitude, frequency, or shape of the oscillating motion. The foil was also found to produce greater thrust than predicted by linear inviscid theory. [2] investigated 1-D heaving only foils both experimentally and numerically. Results compared experimental values to an inviscid, unsteady panel code coupled with a nonlinear wake model and found excellent comparisons over a range of frequencies in both drag and thrust producing regimes proving that thrust production mechanisms are mainly inviscid phenomena. [3] studied a 2-D heaving and pitching foil and found that the efficiency of the thrust produced was dependent on the wake structure behind the foil. Results showed that the highest efficiencies were linked to the formation of a leading-edge vortex on each side per one half-cycle. These leading-edge vortexes interact with the vorticity at the trailing edge in order to form a reverse von Kármán street. Fin propulsion observed in marine animals is a fully three dimensional phenomenon. [4] performed wake visualization of a 1-D pitching foil and found the formation of a three-dimensional von Kármán vortex street at low frequencies but as frequencies increase the wake will split and separate behind the foil. Similar results are found by [5] in their numerical work on low aspect ratio oscillating foils.

In the 3-D rolling and pitching foils, the foil uses a rotary oscillation about a shoulder joint with coupled oscillatory pitching. This configuration has been used as propulsion in vehicles akin to a robotic turtle, [6]. Studies by [7], [8], and [9] identify the Strouhal number, $S t$, and maximum angle of attack, $\alpha_{\max }$, as the primary non-dimensional parameters that govern the change in lift and thrust coefficients of the oscillating foils.

\subsubsection{Effect of the boundary}

Unmanned Underwater Vehicles (UUV) have increasing needs to access more complex areas including operating in near surface and near bottom conditions. The 'ground effect' resulting from operating near a solid boundary has been extensively studied for static foils(see [10] for review). More recently numerical and experimental studies have examined ground effect for oscillating foils (see [11] for a more in depth review). The presence of suction an repulsion forces, as well as increased thrust, is typically reported within less than 2 chord lengths of the wall. The magnitude and direction of the forces perpendicular to the wall 
are determined by the kinematics of the foil as well as the distance between the foil and the solid boundary. Numerical studies by [12] predict ground effect in 1-D heaving foils where the net force is dependent on the distance to a solid boundary. The foil experiences first a suction force and then repulsion as the separating distance decreases. Further investigation reported in [13] found that as the foil approaches the boundary, vortexes will start to be formed and shed from the solid boundary. The vortex pairs begin to stretch and rotate as the separation decreases causing the wake to deflect away from boundary. Experiments by [14] showed that in a flow tank, a pitching only 1-D foil will experience increased thrust while in the presence of a solid boundary with a region of attraction and repulsion. However,in this case the attraction region was located at greater distances from the wall than the repulsion region. Using a 2-D heaving and pitching foil configuration, [15] found that restricting the span-wise flow around the tip of the foil can have a significant effect on the magnitude and direction of forces experienced by the foil and supports the idea of ground effect being an inherently three dimensional phenomenon. In [11], the presence of a solid boundary was shown to effect forces on a 3-D rolling and pitching foil. A non-zero mean lift, indicating an asymmetry in the wake structure, was seen across the entire range of the kinematics tested. The results were compared to [15] and showed that ground effect is a fully three dimensional phenomenon and that extrapolation of 2-D foil studies into 3-D foils would be inherently inaccurate.

Recently, work has been conducted on 2-D heaving and pitching foils near the surface. [16] found a significant effect due to proximity of the free surface. The numerical studies found a nearly linear relationship between thrust and pitch amplitude for a given Strouhal number as well as a decrease in thrust as the foil approaches the surface.

\subsubsection{Contributions}

Near-surface autonomous underwater vehicle (AUV) operations have been of interest to both the military and civilian communities [17]. These operations include oceanographic data collection, mine countermeasures, as well as intelligence, surveillance and reconnaissance(ISR) missions. In the present work we extend the investigation from [11] to operation of a 3-D rolling and pitching foil near the free surface. When operating near the surface, wave generation is bound to have an effect on the forces experienced by a flapping foil. Quantitative and qualitative analysis of near surface operation of oscillating foils can pro- 
vide insight into modeling and improve performance of autonomous vehicles equipped with this type of propulsor.

\subsection{Methodology}

Fluid forces and moments on a foil at varying distance from the free surface were measured for a foil towed in a water tank. The experimental apparatus used in [11] to conduct ground effect tests in the University of Rhode Island tow tank was modified to allow for testing near the surface. Details of the apparatus from [11] are repeated here for completeness. The position of the foil was raised and lowered to allow the foil to flap in the free stream and with increasing proximity of the free surface of the tank.

\subsubsection{Experimental Apparatus}

The foil drive mechanism consists of a two canister oscillating mechanism mounted to an overhead towing carriage using a trio of faired struts, as shown in Figure 2.2.3. The deepest mounting point is approximately $0.5 \mathrm{~m}$ beneath the surface and the foil can be raised by increments of approximately $0.05 \mathrm{~m}$. The tow tank measures $3.65 \mathrm{~m}$ wide and $30 \mathrm{~m}$ long with a maximum depth of $1.55 \mathrm{~m}$. The depth profile in the tank can be adjusted with seven movable bottom panels spanning the width of the tank which were set to the greatest possible depth. This left a $8 \mathrm{~m}$ long test section that allowed for the carriage to ramp up to speed before entering the test zone and avoiding any possible interaction with the adjustable bottom panels that slope upward toward one end of the tank.

\subsubsection{Foil Drive Mechanism}

The test foil is a nominally rigid rectangular planform foil with a constant NACA-0012 cross section and an aspect ratio of 4.2 with a span of $0.398 \mathrm{~m}$ and a chord of $0.095 \mathrm{~m}$ (See Figure 2.2.4). The foil attachment within the pitch canister is shown in cutaway detail in Figure 2.2.5. The pitch motor drive system and the foil shaft bearings are mounted directly to a 6-axis strain-gauge dynamometer (ATI Gamma SI-65-5) installed inside the pitch canister. The foil is mounted directly to the sensor to avoid direct measurement of drag forces on the drive apparatus. A floating dynamic o-ring seal, as detailed in [7], is used to minimize mechanical coupling between the canister and the foil shaft where it exits the housing. 


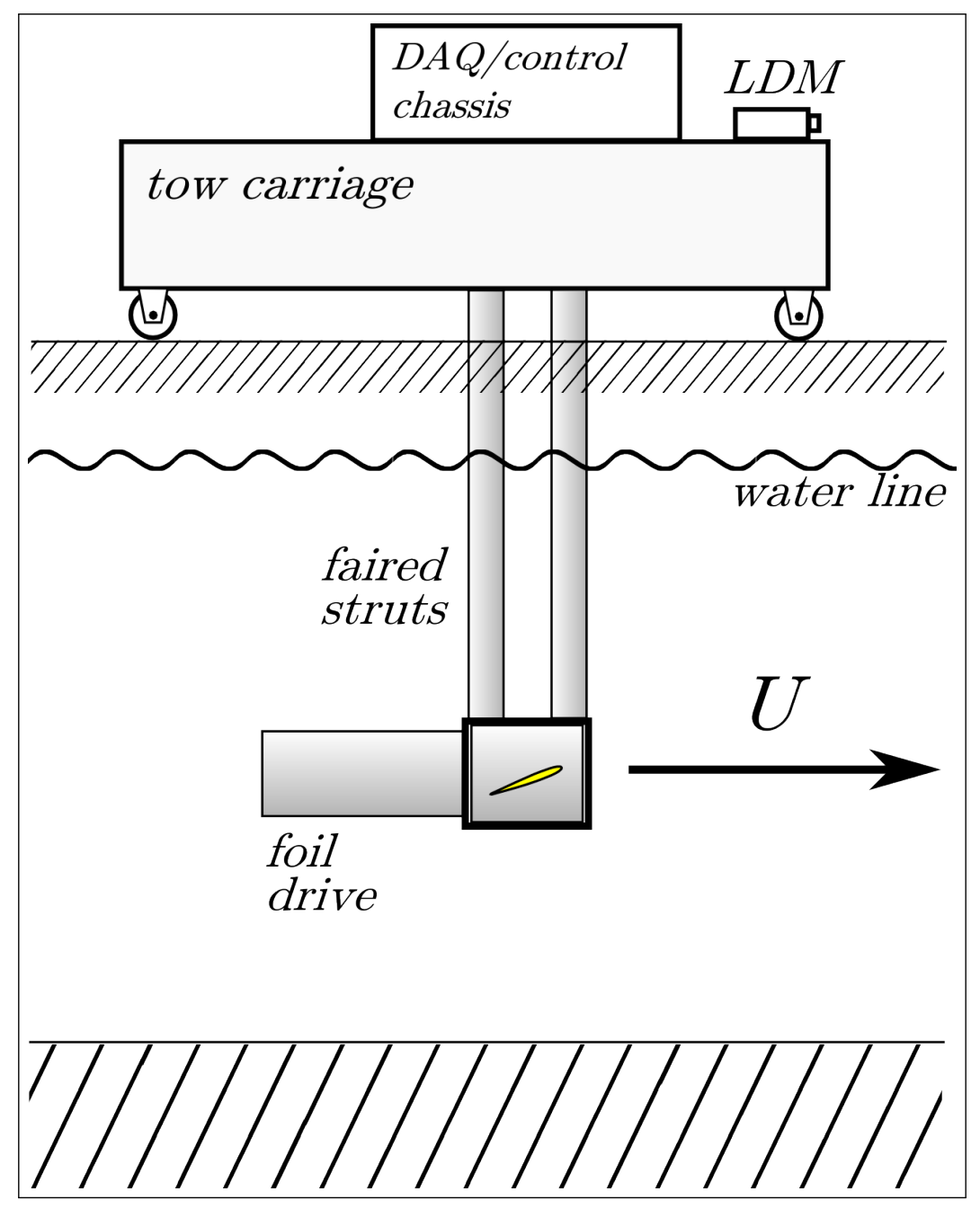

Figure 2.2.1: Side view of foil apparatus as installed in tow tank. DAQ, communications, and foil control are managed through an integrated electronics chassis mounted on the self-propelled tow carriage. The laser distance measurement (LDM) provides real-time measurement of carriage position relative to the end of the tow tank. The roll axis of the foil is parallel to the direction of motion. [11]

The six analog outputs from the dynamometer are amplified and recorded by a DAQ module (National Instrument PXI-6225) installed in a carriage mounted chassis (National Instruments PXIe-1082). The angular position in roll is captured using a string potentiometer wrapped once around the moving canister. Carriage position is logged at $10 \mathrm{~Hz}$ using a laser distance measurement device (Astech LDM42A) mounted to the carriage. The data acquisition hardware and control computer are connect via fiber optics for fast reliable communication. 


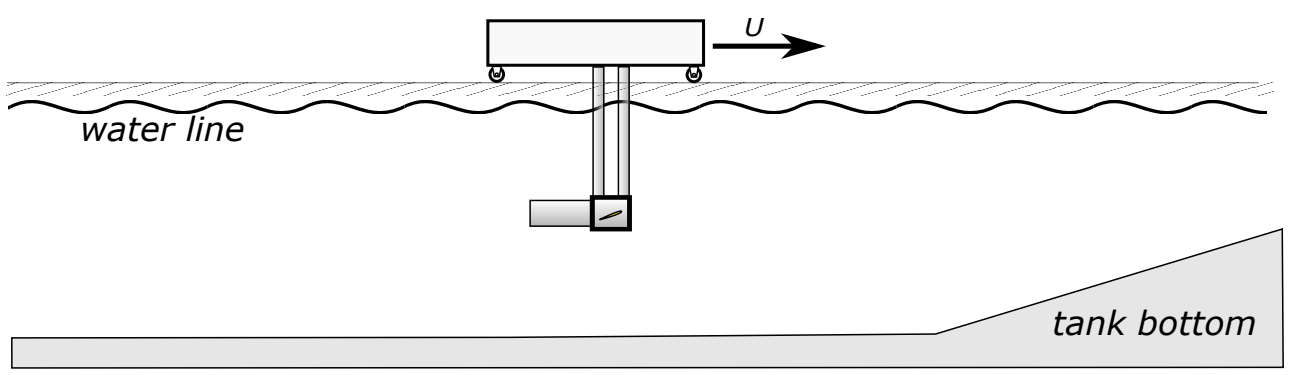

Figure 2.2.2: Side view cross section of the tow tank showing the depth profile created by adjustment of the tank's solid bottom panels. The foil drive is shown in the transition zone between freestream and ground effect test zone, and is shown mounted at a height which results in the closest bottom approach.[11]

\subsubsection{Foil Kinematics}

Table 2.2.1: Experimental Parameters: Constants

\begin{tabular}{ccc}
\hline Carriage velocity & $U$ & $0.5 \frac{\mathrm{m}}{\mathrm{s}}$ \\
Roll amplitude & $\Phi_{0}$ & $12^{\circ}$ \\
Foil span length & $\mathrm{S}$ & $0.398 \mathrm{~m}$ \\
Foil chord length & $\mathrm{c}$ & $0.095 \mathrm{~m}$ \\
Foil planform area & $A_{p}=S c$ & $0.038 \mathrm{~m}^{2}$ \\
Foil aspect ratio & $A R=\frac{S}{c}$ & 4.2 \\
Distance to 70\% span & $r_{0.7}$ & $0.41 \mathrm{~m}$ \\
2-D heave of 70\% span point & $h_{0.7}$ & $0.17 \mathrm{~m}$ \\
Kinematic viscosity, $\mathrm{H}_{2} \mathrm{O}$ & $\nu$ & $10^{-6}$ \\
Reynolds number & $\frac{U c}{\nu}$ & 57,000 \\
\hline
\end{tabular}

Table 2.2.2: Experimental Parameters: Independent Variables

\begin{tabular}{lcc}
\hline Max. nominal angle of attack & $\alpha_{\max }$ & {$[20,25,30,35,40]^{\circ}$} \\
Strouhal number & $S t$ & {$[0.3,0.4,0.5]$} \\
Heave to chord ratio & $\frac{h_{0.7}}{c}$ & 1.8 \\
Depth to chord ratio & $H^{*}=\frac{h}{c}$ & {$[1.75,2.31,2.88,3.44,4.0,4.56,5.68]$} \\
\hline
\end{tabular}

Table 2.2.3: Experimental Parameters: Dependent Variables

\begin{tabular}{ccc}
\hline Pitch amplitude & $\theta_{0}$ & chosen to obtain desired $\alpha_{0}$ \\
Oscillation frequency & $\omega$ & chosen to obtain desired $S t$ \\
\hline
\end{tabular}




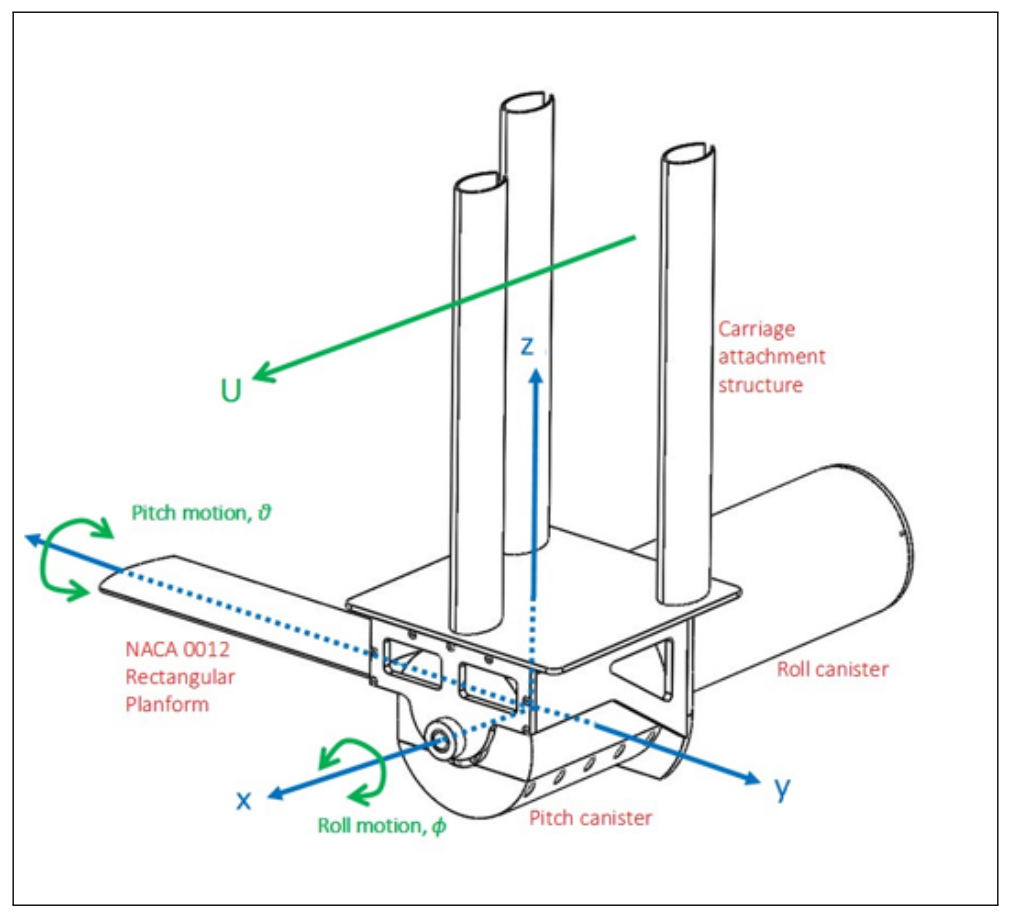

Figure 2.2.3: Foil drive mechanism and attachment. The submersible foil drive apparatus consists of two pressure housings, each containing a DC brush motor to actuate roll or pitch. Drive electronics are contained within the roll canister, while power and communication are supplied from the overhead carriage through underwater connectors. The foil shaft bearings are mounted to a rigid platform supported by a 6-DOF dynamometer inside the pitch canister, as detailed in Figure 2.2.5.[11]

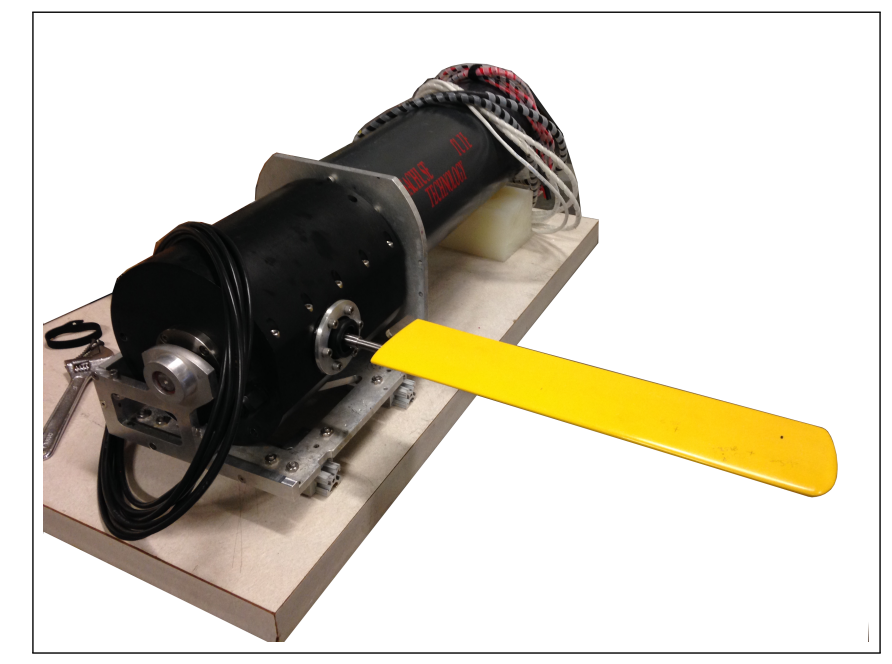

Figure 2.2.4: Image of foil drive apparatus prior to installation with foil mounted in place. The foil has a uniform NACA-0012 cross-section, with span of $0.398 \mathrm{~m}$ and chord of $0.095 \mathrm{~m}$, and is nominally rigid, with a titanium frame and cast outer shape.[11] 


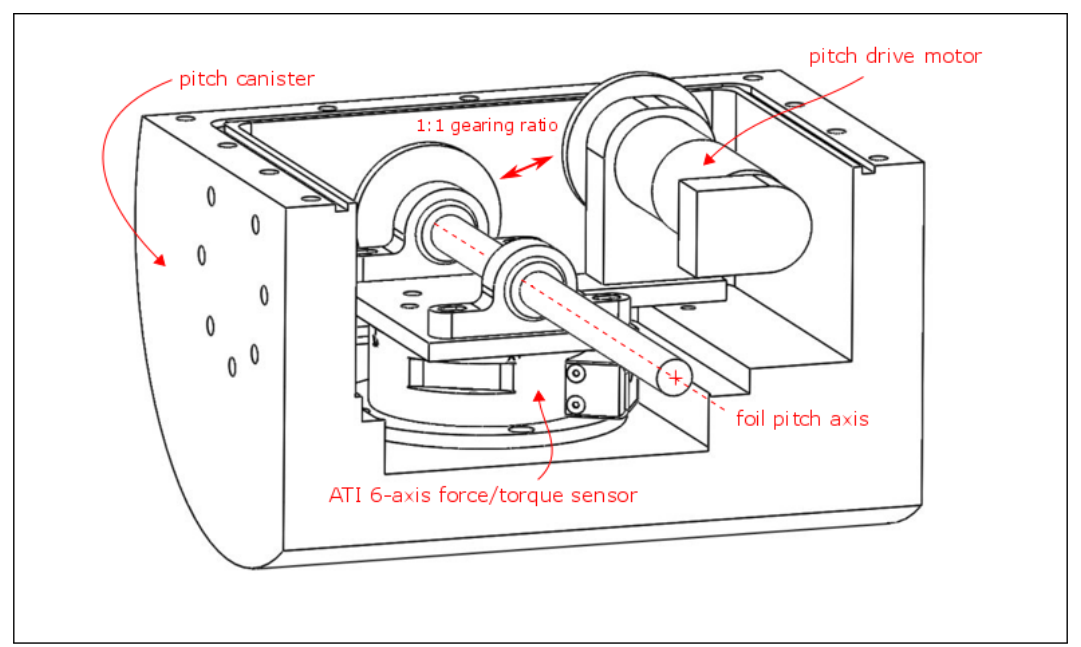

Figure 2.2.5: The pitch canister containing sensor and foil drive train. The front face of the canister is cut away in this view to show internal detail. The motor and shaft bearings are mounted to a rigid platform supported by the six-axis dynamometer. [11]

Sinusoidal pitch and roll trajectories with a $90^{\circ}$ phase offset were used for all experiments. The foil motion can be completely described by (1.1) and (1.2),

$$
\begin{aligned}
& \Phi(t)=\Phi_{0} \sin (\omega t) \\
& \theta(t)=\theta_{0} \cos (\omega t)
\end{aligned}
$$

where $\Phi_{0}$ is the amplitude of the roll motion, and $\theta_{0}$ is the amplitude of the pitch motion. $\Phi$ and $\theta$ are defined to be zero where the foil planform is parallel to the surface plane. The resulting nominal angle of attack as a function of time, $\alpha$, of the foil is given by,

$$
\alpha(t)=-\arctan \left(\frac{\omega r_{0.7} \Phi(t)}{U}\right)+\theta(t)
$$

where $U$ is the carriage speed. By convention, $\alpha$ is calculated at the $70 \%$ chord distance, i.e. at a point $r_{0.7}$ from the origin, however $\alpha$ will vary across the span of the foil due to differences in angular velocity.

The relevant non-dimensional parameters of thrust producing oscillating foils are Strouhal number, $S t=\frac{2 r_{0.7} \Phi_{0} f}{U}$, maximum angle of attack, $\alpha_{\max }$, and the Reynolds number, $R e=\frac{U c}{\nu}$, where $\nu \approx 10^{-6} \mathrm{~m}^{2} \mathrm{~s}^{-1}$ is the kinematic viscosity of water [8]. Furthermore we define an additional independent variable, $H^{*}=\frac{h}{c}$, the non-dimensional distance from the surface boundary, where $h$ is the distance from the free surface to the axis of pitch rotation, following [11] where $h$ represented distance to the solid boundary. 


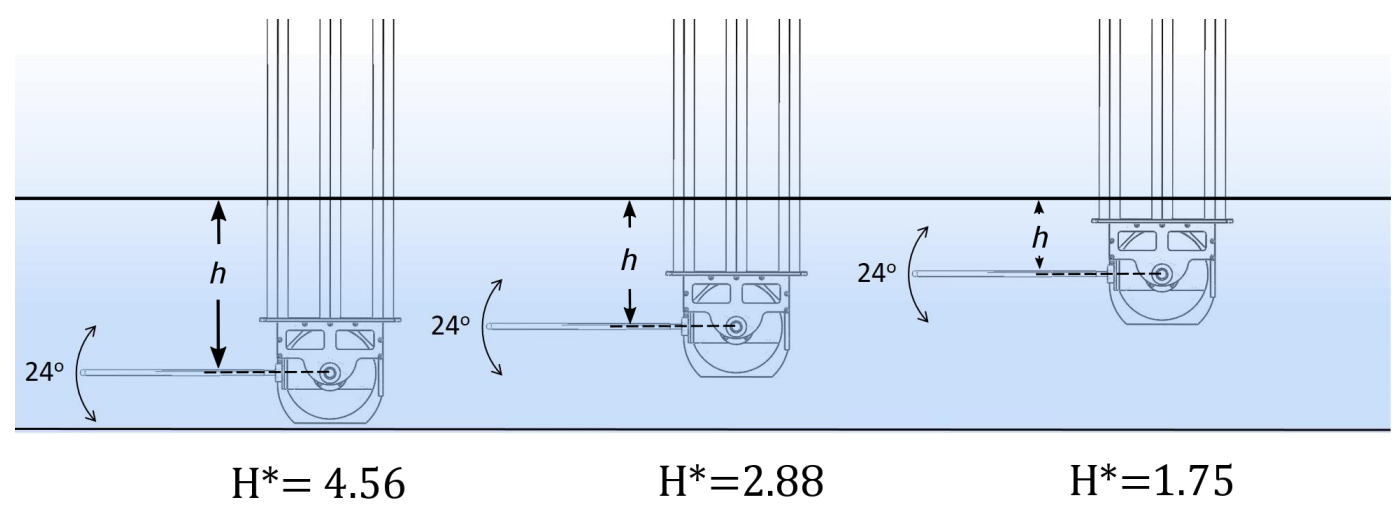

Figure 2.2.6

Figure 2.2.7: The three dimensional foil with different values of $H^{*}$

Reynolds number remained constant in all trials at $R e=57,000$ for a constant carriage speed of $0.5 \frac{\mathrm{m}}{\mathrm{s}}$.

With carriage speed, foil geometry, and roll amplitude all held constant, the desired $S t$ was achieved by varying the oscillating frequency, $f$.

$H^{*}$ was varied by adjusting the distance $h$ (see Figure 2.2.6) through changes in the height of the mount point on the overhead carriage.

For harmonic rolling and pitching, it is evident from equation (1.3) that the evolution of $\alpha(t)$ is not sinusoidal. For a given $\Phi_{0}$ and $U$, an iterative procedure was used to determine the value of $\theta_{0}$ that produced the desired $\alpha_{\max }$.

Trials were conducted for $S t=\left[\begin{array}{lll}0.30 .40 .5\end{array}\right], \alpha_{\max }=\left[20^{\circ} 25^{\circ} 30^{\circ} 35^{\circ} 40^{\circ}\right]$, and $H^{*}=[1.75$ $2.312 .883 .444 .04 .565 .68] . \quad H^{*}=1.75$ was chosen as the minimum operating point to minimize disturbances in the free surface due to the towing apparatus. The kinematic parameter space was chosen to align with previous work in 3-D oscillating foils by [8] and [11] to which we compare the data gathered in this experiment.

\subsubsection{Experimental Procedure}

Before each trial, the carriage is reset to a position against one end of the tank. The 6-axis dynamometer is tared to compensate for the in water weight of the foil before any motion. The foil motion is started, and after three periods elapse, the carriage is accelerated up to $0.5 \frac{m}{s}$ and travels the length of the tank before it is stopped. The foil motion is stopped, the foil is homed to return it to zero roll and zero pitch and finally the carriage is returned to its starting position. Each trial runs for approximately 40 seconds, after which 
the experiment is reset and the tank is allowed to settle for eight minutes before the next trial begins. After all kinematics are run, the foil is raised to a new depth and the testing continues.

\section{$2.3 \quad$ Results}

Results are reported in terms of non-dimensional lift and thrust coefficients, $C_{L}$ and $C_{T}$, respectively, as defined in equations 1.5 and 1.4,

$$
\begin{gathered}
C_{L}=\frac{F_{z}(t)}{0.5 \rho U^{2} S c} \\
C_{T}=\frac{F_{x}(t)}{0.5 \rho U^{2} S c}
\end{gathered}
$$

where $\rho$ is the density of water, $U$ is the carriage speed, and $S$ and $c$ are the foil span and chord lengths. $F_{z}$ is the measured force perpendicular to the span of the foil and incoming flow. $F_{x}$ is the thrust force in line with incoming flow. $\bar{C}_{L}$ and $\overline{C_{T}}$ denote mean lift and thrust coefficients averaged over a complete cycle of motion, and are calculated as follows, for each cycle of motion:

$$
\begin{aligned}
\bar{C}_{T} & =\frac{\int_{\tau_{i}}^{\tau_{i}+T} C_{T}(t) \mathrm{d} t}{T} \\
\bar{C}_{L} & =\frac{\int_{\tau_{i}}^{\tau_{i}+T} C_{L}(t) \mathrm{d} t}{T}
\end{aligned}
$$

where $T$ is the period of the oscillating motion and $\tau_{i}$ is the start of the $i^{\text {th }}$ cycle. 


\subsubsection{Mean thrust in open water}

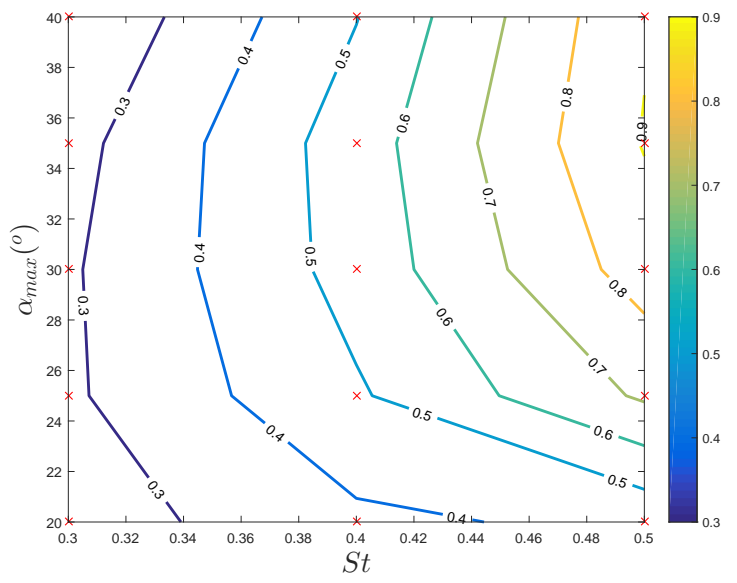

(a) Measured $\overline{C_{T}}$

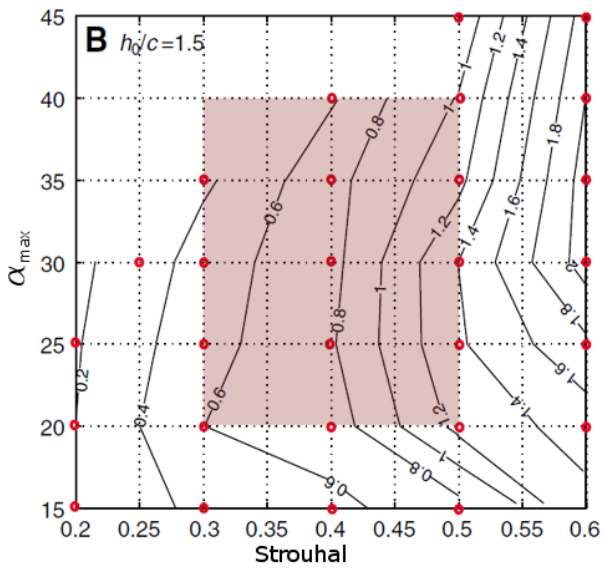

(b) Source: [8]

Figure 2.3.1: (a) Mean freestream thrust coefficient, $\bar{C}_{T}$, from present work, $\frac{h_{0.7}}{c}=1.8$.

(b) $\bar{C}_{T}$ as reported by [8] for a rolling and pitching foil operated with $\frac{h_{o}}{c}=1.5$. The present work covers a subset of the parameter space shown here, as highlighted in red.

Contours of the mean freestream thrust coefficient for all $S t$ and $\alpha_{\max }$ are shown in Figure 2.3.1a. Results show that $\overline{C_{T}}$ increases with $S t$ for a given value of $\alpha_{\max }$, which agrees with previous research results from two dimensional [18] [15] and 3-D oscillating foils [19] [7] [8]. Figure 2.3.1b shows values of $\bar{C}_{T}$ recorded in tests of a similar rolling and pitching foil inside a flow tunnel performed by [8]. [8] reports higher total thrust with lower sensitivity to nominal angle of attack at low values of $\alpha_{\max }$ as expected given the larger distance from axis of roll rotation to the foil base (caused by the use of an in-line force sensor) and the larger aspect ratio of the foil itself. Figure 2.3.2 shows the foil apparatus used for studies in [8]. The dependence of lift coefficients on $S t$ and $\alpha_{\max }$ demonstrates the same trends providing confidence in the validity of the sensing and actuation approach used. 
A

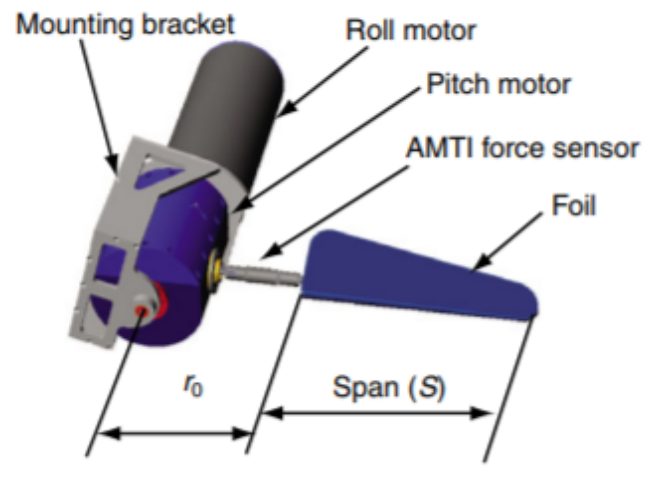

(a)

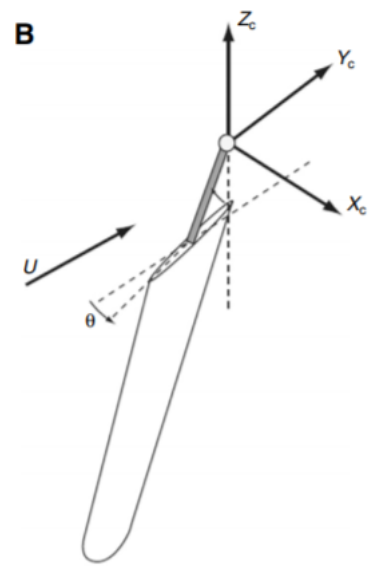

(b)

Figure 2.3.2: (a) Oscillating foil apparatus and (b)Foil shape and orientation in previous work by $[8]$.

\subsubsection{Change in mean lift}

As the foil approaches the surface, there is a change in both the mean lift and mean thrust coefficients. The magnitude of this change is dependent on the kinematic parameters. The magnitude of change, $\Delta \bar{C}_{L}$, is shown in Figure 2.3.3b, which gives contours of the change in $\bar{C}_{L}$ between the freestream and closest approach to the surface. Changes in mean lift are negative across all kinematics tested with the magnitude ranging from $\Delta \overline{C_{L}}=-0.08$ to -0.18. Negative $\Delta \bar{C}_{L}$ indicates a repulsion force, away from the free surface. The greatest change was found at the highest $\alpha_{\max }$ and lowest $S t$, and for each Strouhal number, the magnitude of $\Delta \bar{C}_{L}$ increases with $\alpha_{\max }$. Contours of $\overline{C_{L}}$ during freestream operation are shown in Figure 2.3.3a, to provide a comparison between the magnitude of observed surface effect seen in Figure 2.3.3b and the magnitude of variations in lift caused by mechanical asymmetries in the test apparatus.

\subsubsection{Changes in mean thrust}

Contours of changes in mean thrust coefficient, $\Delta \bar{C}_{T}$, at the closest approach to the surface are shown in Figure 2.3.4a. $\Delta \overline{C_{T}}$ is negative across all tested kinematics and ranges from magnitudes of $3 \%$ to $25 \%$ with the maximum found at low $S t$ and low $\alpha_{\max }$ and minimum at high $S t$ and low $\alpha_{\max }$. For, comparison $\Delta \bar{C}_{T}$ for a foil approaching a solid boundary, [11], is shown in Figure 2.3.4b. In these tests, thrust was increased across all kinematics tested ranging from 2 to 7 percent which is opposite to the current work. The 


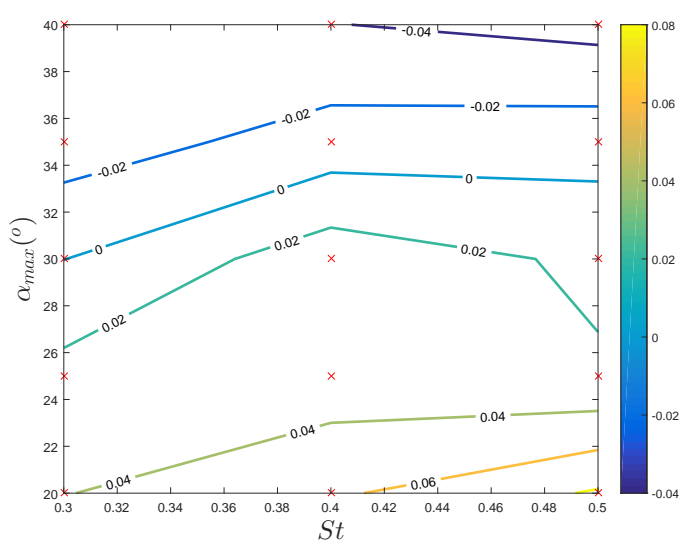

(a) Mean Freestream Lift Coefficient, $\overline{C_{L}}$

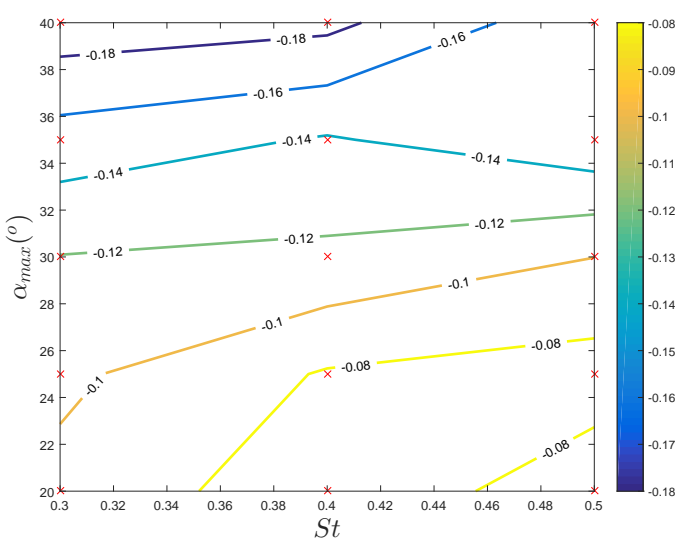

(b) Lift Coefficient Change $\Delta \bar{C}_{L}$

Figure 2.3.3: (a) Mean lift coefficient, $\bar{C}_{L}$, while operating in the freestream. (b)Changes in mean lift coefficient, with the foil operating at the closest approach to the surface $\left(H^{*}=1.75\right)$ vs. operation in the freestream $\left(H^{*}=5.68\right)$.

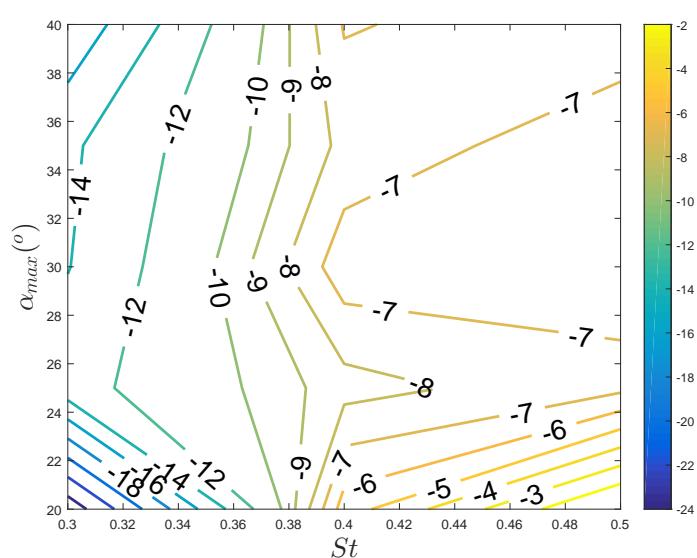

(a)

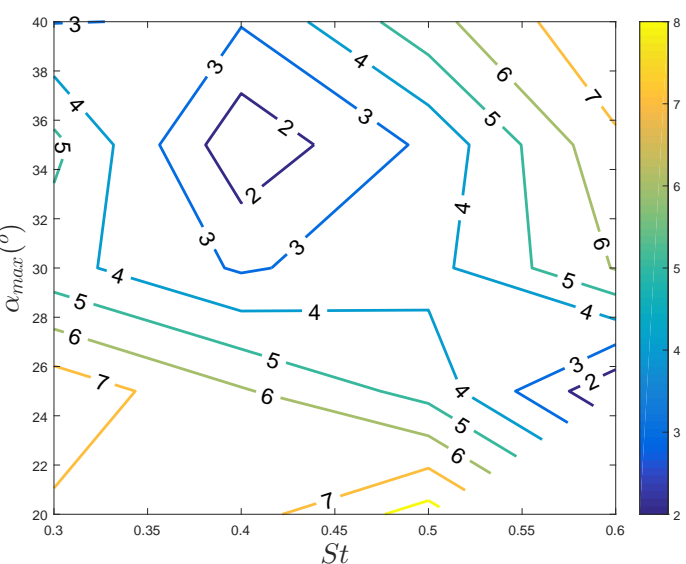

(b)

Figure 2.3.4: Differences in the mean thrust coefficient, $\Delta \bar{C}_{T}$, from (a) current work on the effect of a free surface and (b) previous work on the effect of solid boundary[11], with the foil operating at the closest $\operatorname{approach}\left(H^{*}=1.75\right.$ and 1.4 respectively) to the boundary vs. operation in the freestream. 


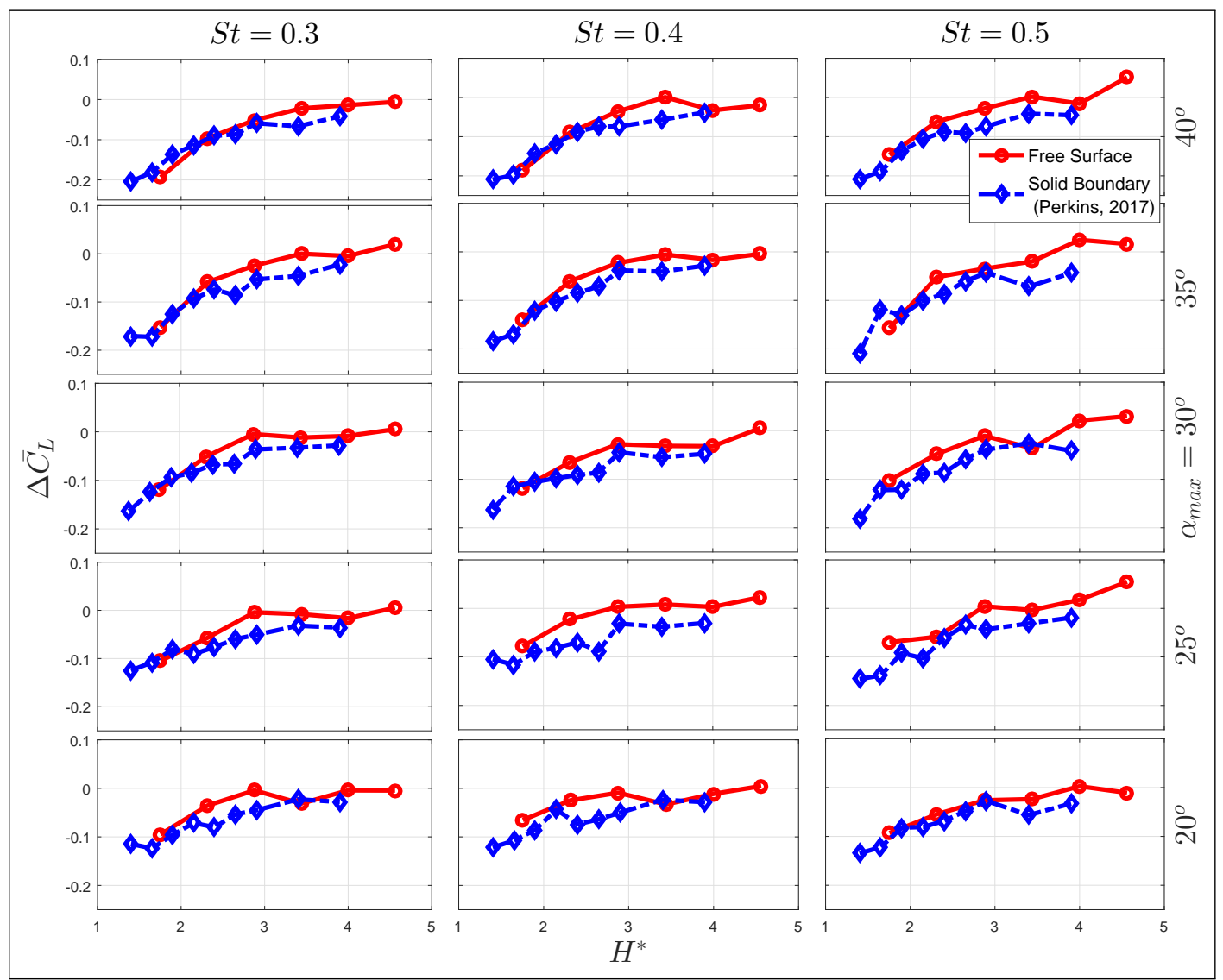

Figure 2.3.5: Comparison of the effect of different boundaries on $\Delta \bar{C}_{L}$ for $S t=[0.3,0.4,0.5]$ and $\alpha_{\max }=\left[20^{\circ}, 25^{\circ}, 30^{\circ}, 35^{\circ}, 40^{\circ}, 45^{\circ}\right]$. Present work, a foil approaching a free surface, compared to results from [11], a foil approaching a solid boundary.

greatest increases were found at high $S t$ and high $\alpha_{\max }$.

\subsubsection{Boundary effect comparison across a range of kinematic parameters.}

Figure 2.3.5 shows the evolution of $\Delta \bar{C}_{L}$ with $H^{*}$ over a range of kinematics for a foil oscillating near a free surface boundary. Results for a foil oscillating near a solid boundary, [11], are shown for comparison. Positive and negative changes correspond to a suction and repulsion force experienced by the foil respectively. As $H^{*}$ decreases, the magnitude of $\Delta \bar{C}_{L}$ increases in all cases for both types of boundaries. The greatest changes in lift occurring at low $S t$ for both boundary types. Furthermore, Figure 2.3.5 shows that relationship between $\Delta \bar{C}_{L}$ and $H^{*}$ is not linear for either case. 


\subsection{Discussion}

The forces on a oscillating foil propulsor are affected by the presence of the free surface.

We highlight the significant conclusions that can be taken from these results below:

- Thrust generating rolling and pitching oscillating foils experience a repulsion force as they approach a free surface.

- Observed effect on lift forces of an oscillating foil are similar for free surface and solid boundaries.

- Thrust production difference can be accounted for by wave generation.

\subsubsection{Thrust generating rolling and pitching oscillating foils experience a re- pulsion force as they approach a free surface.}

Figure 2.3.3b illustrates that across all tested kinematics, the presence of the free surface repels the foil away from the boundary. The non-zero mean lift indicates that there is an asymmetry in the lift production over a complete cycle. As the motion itself is symmetric, the force production must be influenced by the presence of the free surface.

Ground effect can provide vehicles passive boundary avoidance capabilities[11]. This passive avoidance capability is also present as an oscillating foil approaches a free surface boundary. The repulsion force present could be used to detect the boundary and if coupled with an analysis of the thrust forces a vehicle could distinguish a free surface boundary condition from a solid boundary condition.

\subsubsection{Observed effect on lift forces of a foil oscillating are similar for free surface and solid boundaries.}

Figure 2.3.5 clearly illustrates the similarity between the effects solid and free surface boundaries have on a 3-D oscillating foil. As a function of $H^{*}$ across all kinematics tested, the 'ground effect' and 'free surface effect' develop equally as the foil approaches the boundary. The similarity between these two repulsion forces indicates that there are common hydrodynamic mechanisms affecting the lift forces of an oscillating foil. We believe that the development of waves on the free surface is disrupting and draining energy from the vortex street and may account for the decrease in thrust production.

Figure 2.4.1 shows the results of a numerical study done on a 2-D heaving and pitching oscillating foil in the presence of the free surface (2.4.1a) compared to the results of the 


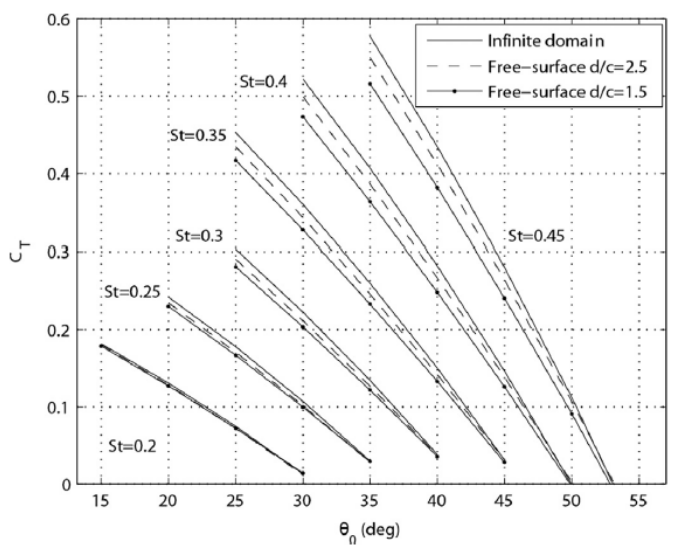

(a) Thrust Coefficient vs Pitch Amplitude [16]

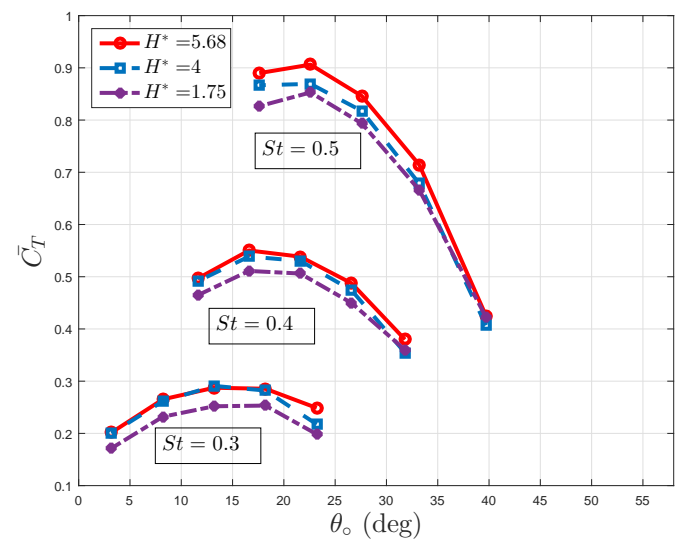

(b) Mean Thrust Coefficient vs Pitch Amplitude (Current Work)

Figure 2.4.1: Numerical study of the effect of a free surface on the thrust coefficient of a 2 -D heaving and pitching oscillating foil, [16] versus the free surface effect on thrust of the current work.

current work (2.4.1b). The numerical studies also show a decrease in thrust due to the surface, but the dependencies on $S t$ and $\theta_{\circ}$ are different but there is little overlap of kinematics tested.

\subsubsection{The difference between ground effect and surface effect on thrust pro- duction can be accounted for by wave generation.}

It is likely that a large portion of the power lost as the oscillating foil approaches the surface is due to wave generation as is common with a body moving near or on the surface. Assuming this to be the case, the power lost due to the surface effect is calculated here by multiplying the thrust difference between the freestream and the closest approach to the surface by the freestream speed (equation 2.8). Equation 2.9 is the power per meter of crest of a deep water wave according to linear wave theory, [20], where $\rho$ is the density of water, $\mathrm{g}$ is gravity, $\mathrm{H}$ is wave height and $\mathrm{f}$ is wave frequency. For these calculations we assume a wave frequency equal to the oscillation frequency of the foil.

$$
\begin{gathered}
P_{\text {loss }}=\left(0.5 \Delta \bar{C}_{T} \rho U^{2} S c\right) U \\
P_{\text {wave }}=\frac{\rho g^{2} H^{2}}{32 \pi f}
\end{gathered}
$$

Figure 2.4.2 shows the estimated wave height in meters of a wave containing all the power lost due to the presence of a free surface. The values range from 6 to 22 centimeters. 


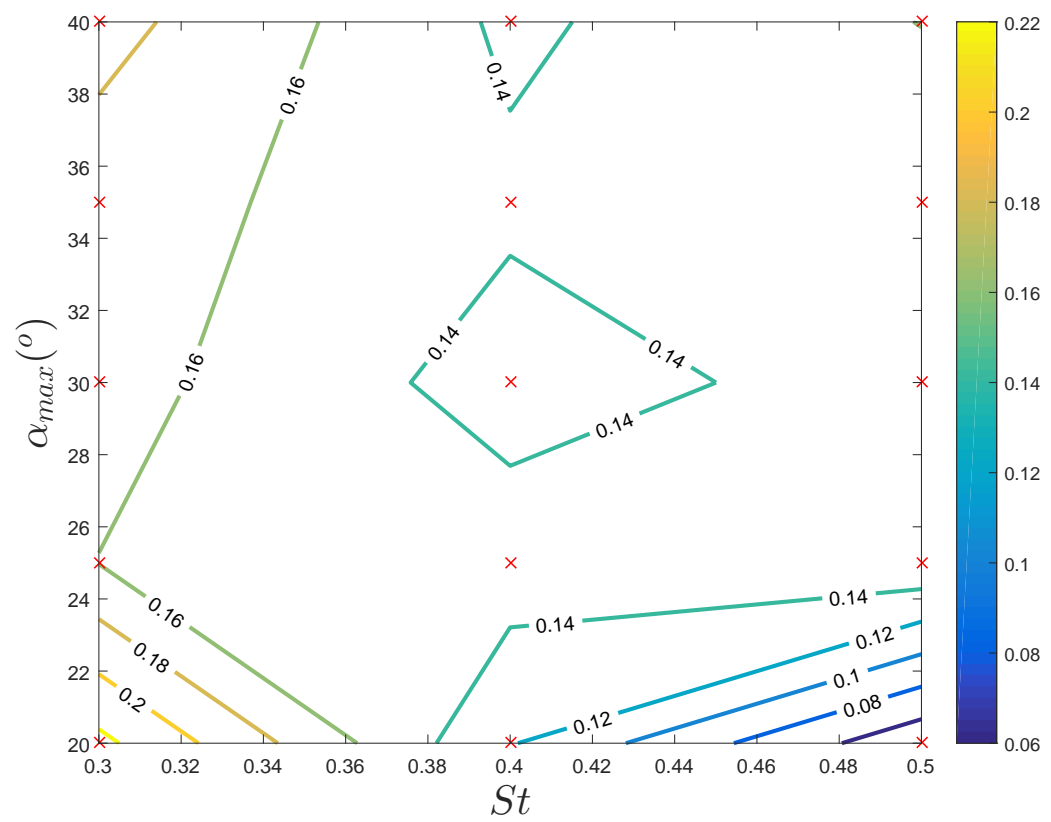

Figure 2.4.2: Wave heights(m) estimated from power loss seen in thrust measurements for the range of tested kinematics at the closest approach to the surface $\left(H^{*}=1.75\right)$

The waves generated during the test were not measured but these estimated values are not unrealistic. It should be emphasized that this is a conservative over-estimation since it is unlikely that $100 \%$ of the power would transfer into waves and the wake created is more complex that a single wave with this wave height. Both gravity and transverse waves are likely to appear in a complex wake pattern with frequencies based on both the oscillation frequency as well as the freestream velocity of the apparatus. The presence of this wave generation at the surface is a large difference from an oscillating foil operating near a solid boundary and can cause the negative effect on thrust production. It is possible that the effect on thrust can be altered by creating constructive or destructive interference between the body waves created by the apparatus passing close to the surface with the wave generated by the flapping motion of the foil, but this has not yet been tested.

\subsection{Conclusion}

The hydrodynamic forces on a 3-D rolling and pitching oscillating foil were characterized at varying distances to the free surface. We conclude that there is a similar effect akin to the previously researched ground effect [11] on a 3-D rolling and pitching oscillating foil when it operates in close proximity to a free surface. The repulsion force is present in similar magnitudes, however the change in mean thrust is negative as opposed to the increase in 
thrust seen by a foil operating in ground effect. This difference in thrust is likely due to wave generation on the free surface.

Direct extensions of this research include flow visualization to help understand the boundary vortex interactions that affect the force production of the foil. Three dimensional visualization of this flow could shed light on effects due to both solid and free surface boundaries and may in turn give insight into different boundary types. Future research may also investigate how softer compliant materials will interact with the boundaries and if the repulsion force will differ. These experiments provide direct experimental data to inform future design of underwater vehicle propulsion and sensor systems. The effect studied here could be used to not only detect different boundaries but classify and estimate distances to them. As Computational Fluid Dynamics (CFD) improve, this data set will also provide reference data for numerical analyses of 3-D rolling and pitching oscillating foils. We hope that these experiments will provide useful data for further validations and investigations both numerical and experimental into force production of oscillating foils.

\section{List of References}

[1] M. M. Koochesfahani, "Vortical patterns in the wake of an oscillating airfoil," American Institute of Aeronautics and Astronautics, vol. 27, no. 9, pp. 1200-1205, Sept 1989.

[2] K. D. Jones, C. M. Dohring, and M. F. Platzer, "Experimental and Computational Investigation of the Knoller-Betz Effect," AIAA Journal, vol. 36, no. 7, pp. 1240-1246, July 1998.

[3] J. M. Anderson, K. Streitlien, D. S. Barrett, and M. S. Triantafyllou, "Oscillating foils of high propulsive efficiency," Journal of Fluid Mechanics, vol. 360, pp. 41-72, Apr. 1998.

[4] J. H. J. Buchholz and A. J. Smits, "On the evolution of the wake structure produced by a low-aspect-ratio pitching panel," Journal of Fluid Mechanics, vol. 546, no. -1, p. 433, Dec. 2005.

[5] P. Blondeaux, F. Fornarelli, L. Guglielmini, M. S. Triantafyllou, and R. Verzicco, "Numerical experiments on flapping foils mimicking fish-like locomotion," Physics of Fluids, vol. 17, no. 11, p. 113601, Nov. 2005.

[6] S. Licht, V. Polidoro, M. Flores, F. Hover, and M. Triantafyllou, "Design and Projected Performance of a Flapping Foil AUV," IEEE Journal of Oceanic Engineering, vol. 29, no. 3, pp. 786-794, July 2004.

[7] V. Polidoro, "Flapping foil propulsion for cruising and hovering autonomous underwater vehicles," Master's thesis, Massachusetts Institute of Technology, 2003.

[8] A. H. Techet, "Propulsive performance of biologically inspired flapping foils at high Reynolds numbers," Journal of Experimental Biology, vol. 211, no. 2, pp. 274-279, Jan. 2008. 
[9] D. Beal, H. Leinhos, A. Fredette, and R. Berube, "Unified Scaling for Flapping Fins," IEEE Journal of Oceanic Engineering, vol. 38, no. 1, pp. 1-11, 2013.

[10] E. Cui and X. Zhang, "Ground effect aerodynamics," Encyclopedia of Aerospace Engineering, vol. 1, no. Part 3, pp. 245-256, 2010.

[11] M. Perkins, D. Elles, G. Badlissi, A. Mivehchi, J. Dahl, and S. Licht, "Rolling and pitching oscillating foil propulsion in ground effect," Bioinspiration 63 biomimetics, vol. 13, no. 1, p. 016003, 2017.

[12] L. Dai, G. He, and X. Zhang, "Self-propulsion of a Flexible Plunging Foil Near a Solid Wall," Procedia Engineering, vol. 126, pp. 431-435, 2015.

[13] L. Dai, G. He, and X. Zhang, "Self-propelled swimming of a flexible plunging foil near a solid wall," Bioinspiration \& Biomimetics, vol. 11, no. 4, p. 046005, July 2016.

[14] D. B. Quinn and A. J. Smits, "Rectangular panels undergoing pitch oscillation in ground effect," in The 1000 Island Fluids Meeting, 2012.

[15] A. Mivehchi, J. Dahl, and S. Licht, "Heaving and pitching oscillating foil propulsion in ground effect," Journal of Fluids and Structures, vol. 63, pp. 174-187, May 2016.

[16] E. Filippas and K. Belibassakis, "Hydrodynamic analysis of flapping-foil thrusters operating beneath the free surface and in waves," Engineering Analysis with Boundary Elements, vol. 41, pp. 47-59, Apr. 2014.

[17] J.-s. Shen, X.-c. Zhou, and H.-g. Zhang, "Research on Sliding Mode Control for NearSurface AUV Depth Regulation in Waves Circumstance," in 2010 International Conference on Electrical and Control Engineering. Wuhan, China: IEEE, June 2010, pp. $1760-1764$.

[18] D. A. Read, "Oscillating foils for propulsion and maneuvering of ships and underwater vehicles," Master's thesis, Massachusetts Institute of Technology, 2001.

[19] M. D. Flores, "Flapping motion of a three-dimensional foil for propulsion and maneuvering of underwater vehicles," Ph.D. dissertation, Massachusetts Institute of Technology, 2003.

[20] R. G. Dean and R. A. Dalrymple, Water wave mechanics for engineers and scientists. World Scientific Publishing Company, 1991, vol. 2. 


\section{MANUSCRIPT 3}

\section{Combination of force feedback and an analytic flapping foil model to improve control of a flapping foils in the presence of crossflow}

In this paper we combine an analytical model to design a control system to use force feedback from a rolling and pitching oscillating foil to compensate for and estimate the angle of crossflow. An analytic model was used to produce predictive force estimates of multiple oscillating foil systems, across a range of kinematics, and in the presence of crossflow. These predicted forces were compared with experimental data in order to validate the implementation of the analytic model. The comparison proved the code developed was able to accurately replicate previously published results, accurately predict forces on oscillating foil configurations at NUWC-NPT and URI, predict mean force coefficients over a range of foil kinematics and accurately estimate the effect that crossflow will have on the forces experienced by an oscillating foil. Once the code was validated, it was used to design and simulate a simple control system. The control system was implemented on the foil system at URI and was able to compensate and coupled with information from the model, estimate the crossflow angle within a half of a degree.

\subsection{Introduction and Background}

The ocean is a complex environment and navigating it has an endless list of challenges to overcome. New vehicles are being designed that have biomimetic propulsion to improve the maneuverability and agility of these platforms. With these new types of propulsion, the vehicles will need new control systems to allow for accurate thrust and lift production while operating. Oscillating foils in particular have been implemented in underwater vehicles and their control systems are being researched to improve the performance of the foils.

\subsubsection{Oscillating Foils}

Self-propelled underwater vehicles using oscillating foils can be seen in [1] and [2]. These vehicles use coupled rolling and pitching motions to induce a flow across a foil providing lift which can be vectored to produce thrust on a vehicle. More in depth descriptions of different oscillating foil configurations can be found in [3]. 


\subsubsection{Digital Control Theory}

Control of a system starts with the creation of a representative model of the system. A continuous time state space model or 'plant' can be described by the equations in (3.1) where matrices $\mathrm{A}, \mathrm{B}, \mathrm{C}$, and $\mathrm{D}$ are derived from the system.

$$
\begin{aligned}
& \dot{x}=\mathbf{A} x+\mathbf{B} u \\
& y=\mathbf{C} x+\mathbf{D} u
\end{aligned}
$$

For most control applications, however, a computer is necessary and therefore the continuous model needs to be transformed into a discrete time model. A continuous time state space model can be converted to a discrete time or zero-order hold $(\mathrm{ZOH})$ model with the equations shown below in (3.2) and (3.3).

$$
\begin{array}{r}
\boldsymbol{\Phi}=e^{\mathbf{A} T}, \quad \boldsymbol{\Gamma}=\int_{0}^{T} e^{\mathbf{A} \tau} \mathbf{B} d \tau \\
x[k+1]=\mathbf{\Phi} x[k]+\boldsymbol{\Gamma} u[k] \\
y[k]=\mathbf{C} x[k]+\mathbf{D} u[k]
\end{array}
$$

where $\mathrm{k}$ is the time index and $\mathrm{T}$ is the sampling period. Before designing a controller model must also analyzed to ensure it was controllable. To determine if a discrete model is controllable, first the continuous time model must be controllable. This can be checked for a second-order system by forming the controllability matrix as described in (3.4)[4].

$$
W_{c}=[\mathbf{A} \quad \mathbf{A B}]
$$

If the rank of the controllability matrix is equal to the order of the system, than it is controllable. The rank of the controllability matrix was equal to the order of the system and therefore, it is controllable, or there exists a inputs signal that will bring the system from any initial condition to any final condition in a finite amount of time [4]. If the continuous system is controllable, the discrete time is controllable if

$$
T<\frac{\pi}{\beta_{\max }}
$$

where $\mathrm{T}$ is the sampling interval and $\beta_{\max }$ is imaginary part of the pole with the greatest magnitude. 


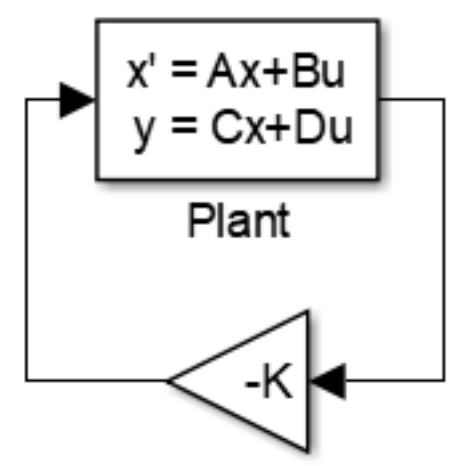

Figure 3.1.1: Continuous time state feedback regulator with continuous time gain matrix $\mathrm{K}$.

A common control method used in digital control is the creation of state feedback regulator as illustrated in Figure 3.1.1. A regulator drives all state variables to zero. The system feeds the current states back through a gain matrix to create the input for the next step of the system. This changes the system from open loop as described by (3.3) to a closed loop system which is mathematically represented by

$$
\begin{array}{r}
x[k+1]=\boldsymbol{\Phi} x[k]+\boldsymbol{\Gamma} u[k] \\
u[k]=-\mathbf{L} x[k]
\end{array}
$$

There are many methods of calculating the the gain matrix, $L$, for a regulator. This paper proposes using pole placement through state feedback using an iterative algorithm. The iterative algorithm will calculate the gain to make the poles of the closed loop system equal to normalized bessel poles as described [4] while ensuring that the eigenvectors are as orthogonal as possible. Moving the poles to these location should provide a desirable system response driving the states of the system to zero with minimal overshoot.

\subsubsection{Contributions}

As biomimetic underwater propulsion evolves, new tools are needed to help improve design and control of new systems. In the present work we replicate results of a previously derived model for forces on a flapping foil and expand on validation cases of the model. We then use the model to design a control system for use on a flapping foil. The control system is tested validating the model as a useful design tool for flapping foil control systems. 


\subsubsection{Useful Definitions}

Results are reported in terms of non-dimensional coefficients as defined

$$
\begin{gathered}
C_{L \alpha}=\frac{F_{z}(t)}{1 / 2 \rho U^{2} S c \sin \alpha} \\
C_{D \alpha}=\frac{F_{x}(t)}{1 / 2 \rho U^{2} S c \sin \alpha} \\
C_{\Gamma \alpha}=\frac{\Gamma_{m i d}(t)}{2 U S \sin \alpha} \\
C_{L}=\frac{F_{z}(t)}{1 / 2 \rho U^{2} S c} \\
C_{T}=\frac{F_{x}(t)}{1 / 2 \rho U^{2} S c}
\end{gathered}
$$

where $\rho$ is the density of water, $U$ is the carriage speed, $\alpha$ is the maximum angle of attack, and $S$ and $c$ are the foil span and chord lengths. $F_{z}$ is the measured force perpendicular to the span of the foil and incoming flow. $F_{x}$ is the thrust force in line with incoming flow. $\bar{C}_{L}$ and $\overline{C_{T}}$ denote mean lift and thrust coefficients averaged over a complete cycle of motion, and are calculated as follows, for each cycle of motion:

$$
\begin{aligned}
\bar{C}_{T} & =\frac{\int_{\tau_{i}}^{\tau_{i}+T} C_{T}(t) \mathrm{d} t}{T} \\
\bar{C}_{L} & =\frac{\int_{\tau_{i}}^{\tau_{i}+T} C_{L}(t) \mathrm{d} t}{T}
\end{aligned}
$$

where $T$ is the period of the oscillating motion and $\tau_{i}$ is the start of the $i^{t h}$ cycle.

\subsection{Model Implementation}

An analytic model has recently been developed at the Massachusetts Institute of Technology (MIT) to predict forces on wings of finite aspect ratio. Detailed in [5], this model is suited for rapid modeling of forces on a flapping foil with the goal of improving on current methods such as computational fluid dynamics (CFD) or unsteady vortex lattice methods (UVLM). CFD simulations are physically accurate, however, due to their complexity, they are computationally slow. UVLM is faster than full CFD simulations, however, they grow slower as the entire wake history is recorded. Research into reduced order modeling has 


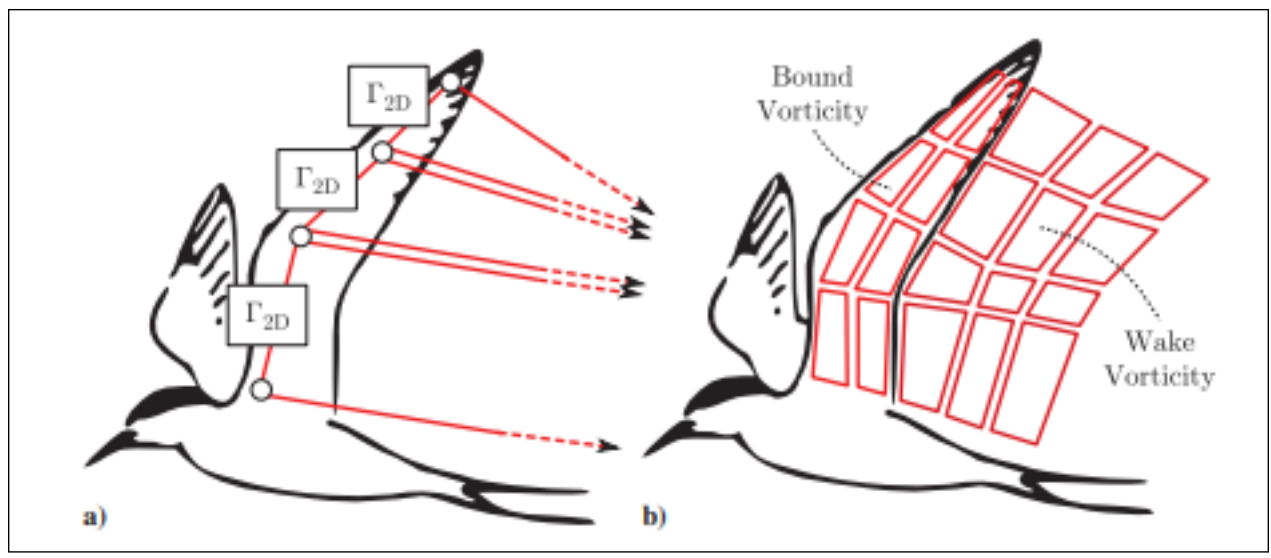

Figure 3.2.1: a.) Proposed state-space wake approximation b.) standard UVLM wake representation for wings [5]

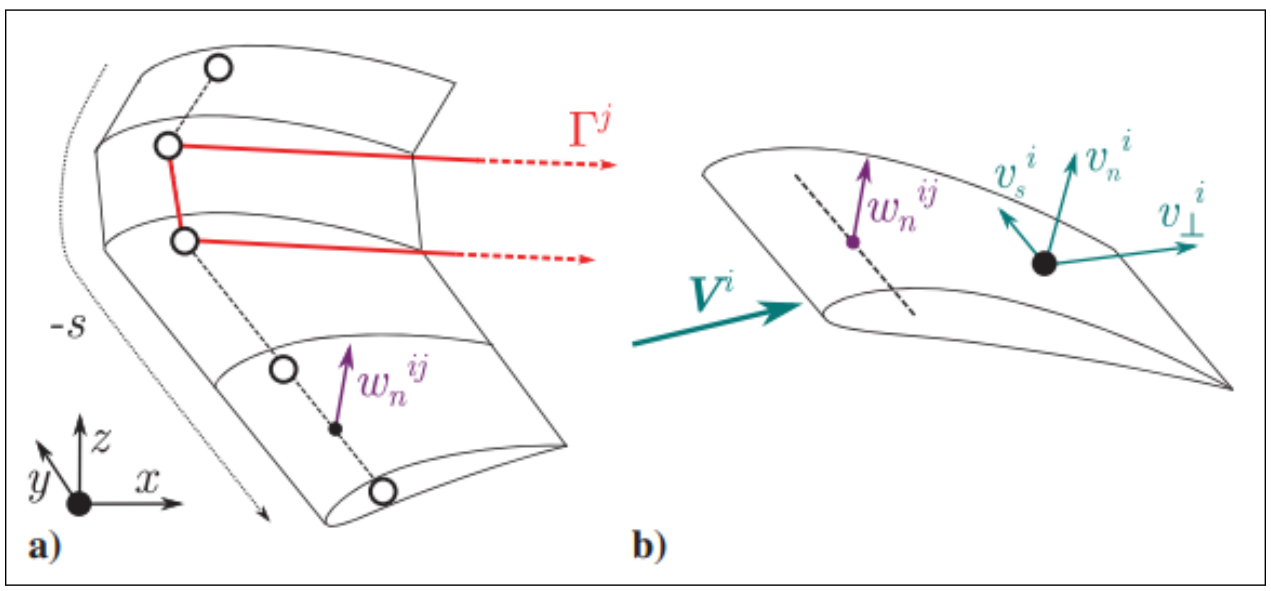

Figure 3.2.2: Discretized wing showing blade elements, horseshoe vortex attachment points as well as reference frames for downwash and velocity vectors. [5] 
found that the dynamics of unsteady wings are highly complicated but simulation outputs such as lift, circulation, and downwash can be modeled more easily [5]. This model presents a fast and computationally cheap solution to model unsteady flapping motion with the goal of aiding active control of oscillating propulsive foils.

Commonly, the wake of an oscillating foil is modeled as a continuous vortex sheet without viscous effects and lacking leading edge vortex (LEV) shedding. The model presented in [5] proposes a novel apprach that approximates the wake as sum of horseshoe vortices and state-space systems of the local wake history. Figure 3.2.1 illustrates the difference between the new wake approximation (a) and the common UVLM representation (b). The wing is separated into several discrete blade elements with a horseshoe vortex overlaid upon each section and trailers of these vortices extending away from the wing tangent to the instantaneous velocity at the point of attachment to the foil. Figure 3.2.2 shows the horseshoe vortex along with the downwash and velocity vectors of each blade element. Low order state-space systems are created for each section in order to track the unsteady circulation and lift of each discrete section providing a local history in place of a full time history. In total, the wake model is a combination of fully unsteady $2-\mathrm{D}$ wake in the state-space models and a quasi-steady 3-D wake that couples the 2-D wakes of each blade element to its neighbors. The model uses the indicial method to obtain the state-space representations of the blade element systems similarly to [6]. [5] proposes two options using 1 state per blade element and 6 states per blade element to record the independent lift and circulation. An additional small downwash correction is provided to consolidate the true 3-D unsteady wake with the previously mentioned quasi- steady and 2-D unsteady wakes.

Using this model, at the University of Rhode Island (URI) we were able to develop a code to calculate the forces on a foil. Following [5], each time step in the model follows this sequence:

1. The circulation is determined by solving for the trailers and circulation states that represent the unsteady wake.

2. The instantaneous downwash is determined from the previously found circulation and the incident velocities are modified.

3. The lift is found from a combination of the downwash and lift states.

4. The time rate of change of all states are calculated, then integrated to give an updated 
state.

After reviewing the paper we decided to use the proposed reduced order state-space system method which includes simplifications allowing for one state per blade element to track both the lift and circulation of that element. We did not add the small additional downwash correction since the correction had little affect on the resultant lift forces and the additional states would have added computational cost. Finally, our model simulations were done in MATLAB R2015 without the use of the Drake toolbox mentioned in [5] and any added mass forces were neglected.

\subsubsection{Result Replication}

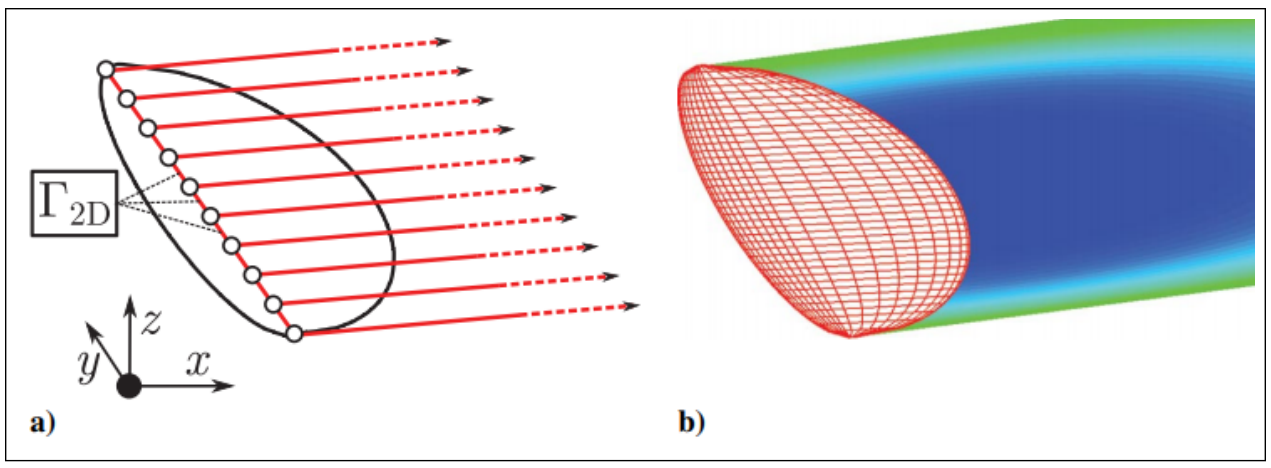

Figure 3.2.3: The elliptical planform wing used in the validation cases with (a) the new proposed wake representation and (b) the UVLM representation. [5]

[5] provides multiple validation cases in order to compare the new model to earlier work. The code developed at URI was used to replicate the results of two of these cases to validate that the code was working properly. The two cases were the impulsive start of a foil from rest and the case of coupled heaving and pitching motions of the same foil. The foil, shown in Figure 3.2.3, is an elliptical planform wing with an aspect ratio of 3, altered to give a straight $\frac{1}{4}$ chord line. The pitch oscillations will rotate about this $\frac{1}{4}$ chord line.

The first case replicated was that of the elliptical foil in an impulsively started flow. The results from [5] are shown in Figure 3.2.4a and the results from our own simulations are show in Figure 3.2.4b. The results of second case with the foil undergoing heaving and pitching motions are shown in Figure 3.2.5. In both cases the results from the code developed at URI are very similar to those published in [5]. There are some small variations during the first few time steps as the states are settling. The reason for these discrepancies 
are unknown at this time but should not affect the final results. These figures show that our code is working properly and can be used in further research.

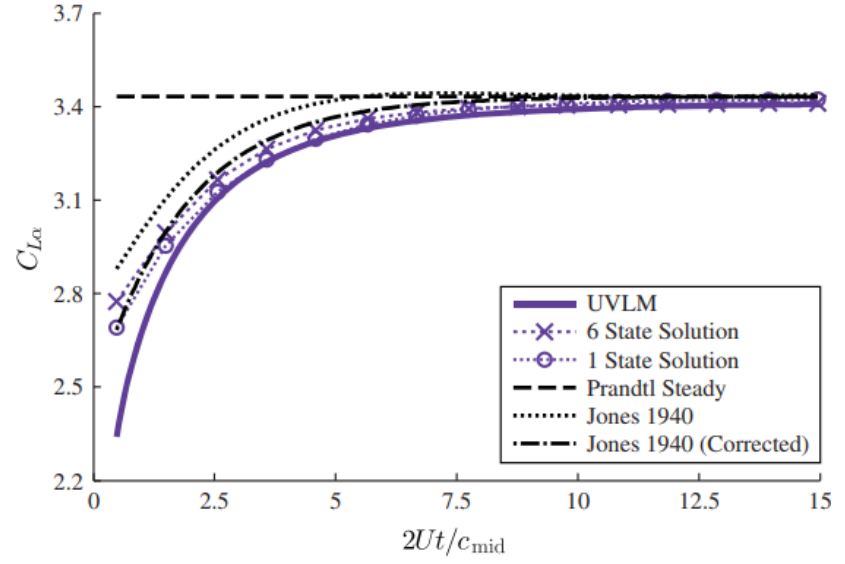

(a)

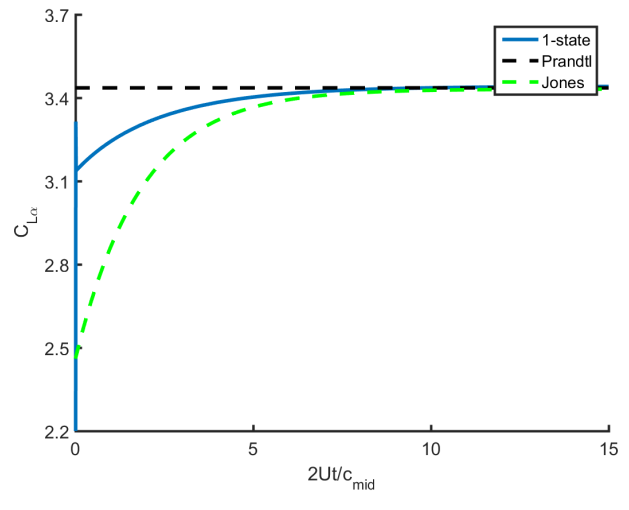

(b)

Figure 3.2.4: Simulated lift on an impulsively started elliptical foil from (a) [5] and (b) the code developed at URI

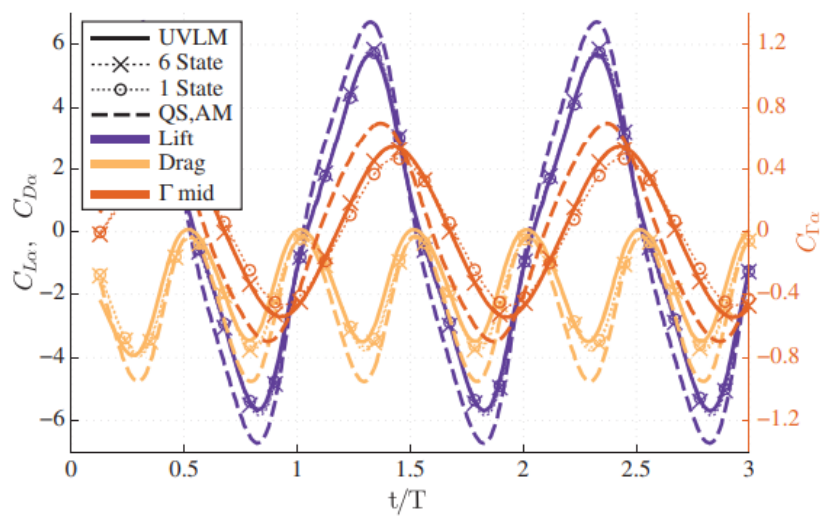

(a)

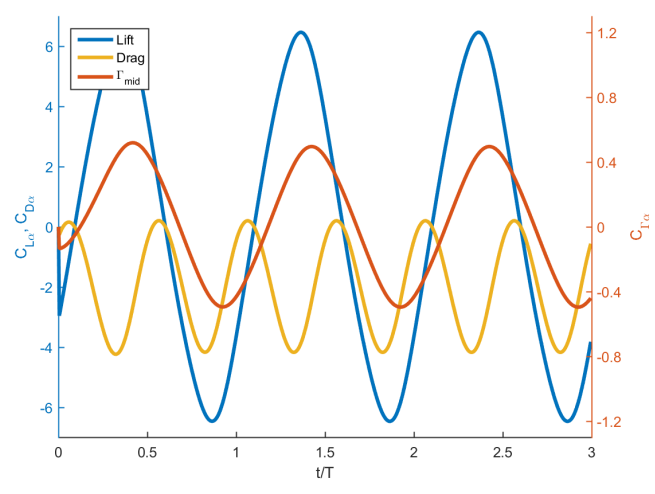

(b)

Figure 3.2.5: Simulation outputs of a heaving and pitching elliptical foil from (a) [5] and (b) the code developed at URI

\subsection{Evaluating Model Accuracy}

After the model code was completed, and the results from the paper were replicated, the model was validated against experimental force data taken from 3-D rolling and pitching foils. The data was taken from two different setups; one at the University of Rhode Island (URI) and another at the Naval Undersea Warefare Center- Newport (NUWC-NPT). The model was compared to force data from both of these apparatuses and under different 
conditions. At URI we were able to compare the accuracy of the model over a range of operating kinematics and at NUWC-NPT were able to the test the model against a foil in the presence of a crossflow and with non-zero pitch biases. The experimental apparatuses of each location are described in Section 3.3.1.

\subsubsection{Experimental Apparatuses}

This section details the oscillating foil configurations and experimental methods of the tests run at each location.

\subsubsection{University of Rhode Island}

The foil drive mechanism consists of a two canister oscillating mechanism mounted to an overhead towing carriage using a trio of faired struts, as shown in Figure 3.3.3. The tow tank measures $3.65 \mathrm{~m}$ wide and $30 \mathrm{~m}$ long with a maximum depth of $1.55 \mathrm{~m}$. The depth profile in the tank can be adjusted with seven movable bottom panels spanning the width of the tank which were set to the greatest possible depth. This left a $8 \mathrm{~m}$ long section that allowed for the carriage to ramp up to speed before entering the test zone and avoiding any possible interaction with the adjustable bottom panels that slope upward toward one end of the tank.

The test foil is a nominally rigid rectangular planform foil with a constant NACA-0012 cross section and an aspect ratio of 4.2 with a span of $0.398 \mathrm{~m}$ and a chord of $0.095 \mathrm{~m}$ (See Figure 3.3.4). The foil attachment within the pitch canister is shown in cutaway detail in Figure 3.3.5. The pitch motor drive system and the foil shaft bearings are mounted directly to a 6-axis strain-gauge dynamometer (ATI Gamma SI-65-5) installed inside the pitch canister. The foil is mounted directly to the sensor to avoid direct measurement of drag forces on the drive apparatus. A floating dynamic o-ring seal, as detailed in [7], is used to minimize mechanical coupling between the canister and the foil shaft where it exits the housing.

The six analog outputs from the dynamometer are amplified and recorded by a DAQ module (National Instrument PXI-6225) installed in a carriage mounted chassis (National Instruments PXIe-1082). The angular position in roll is captured using a string potentiometer wrapped once around the moving canister. Carriage position is logged at $10 \mathrm{~Hz}$ using a laser distance measurement device (Astech LDM42A) mounted to the carriage. The 


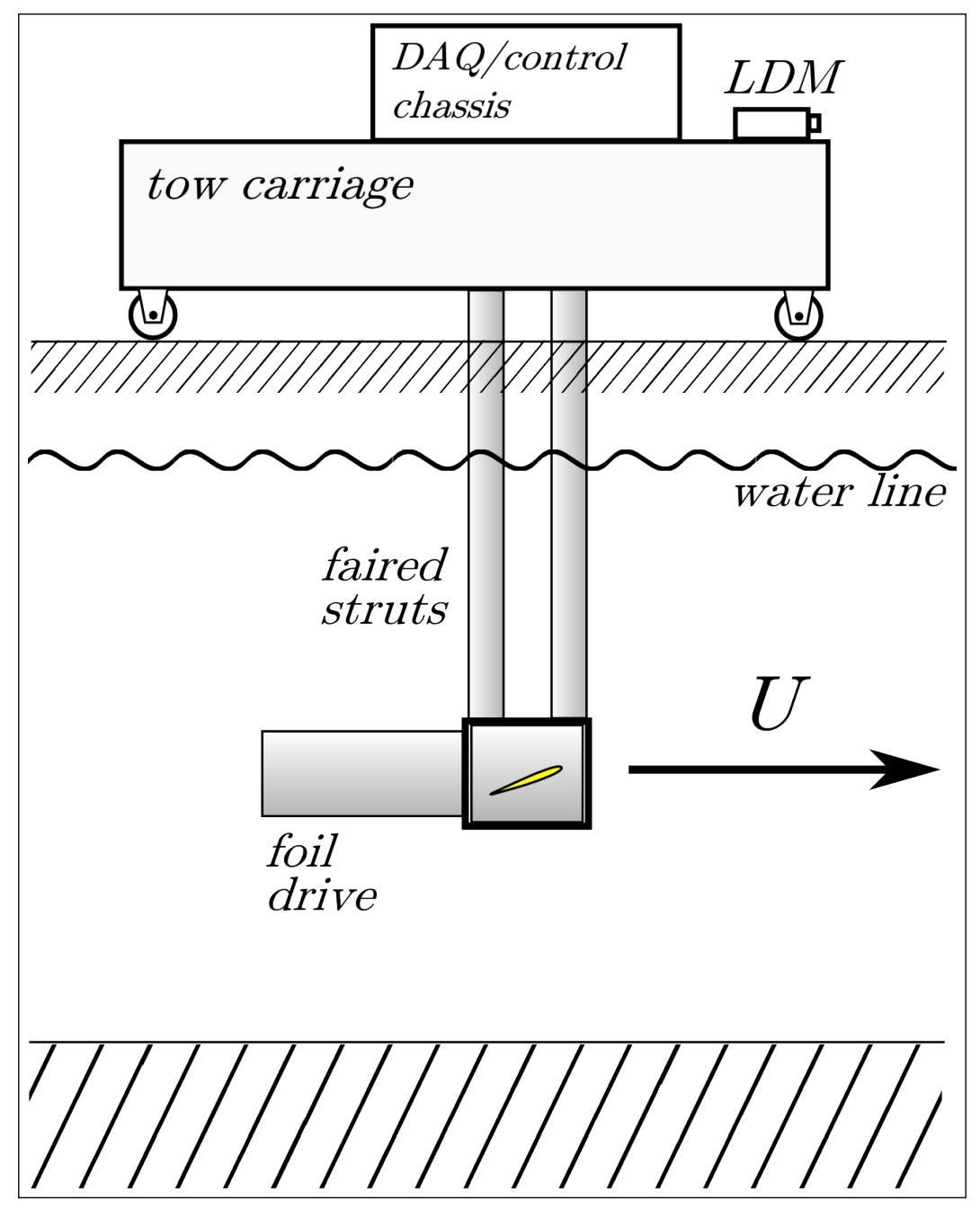

Figure 3.3.1: Side view of foil apparatus as installed in tow tank. DAQ, communications, and foil control are managed through an integrated electronics chassis mounted on the self-propelled tow carriage. The laser distance measurement (LDM) provides real-time measurement of carriage position relative to the end of the tow tank. The roll axis of the foil is parallel to the direction of motion. [3]

data acquisition hardware and control computer are connect via fiber optics for fast reliable communication.

Sinusoidal pitch and roll trajectories with a $90^{\circ}$ phase offset were used for all experiments. The foil motion can be completely described by (3.14) and (3.15),

$$
\begin{aligned}
& \Phi(t)=\Phi_{0} \sin (\omega t) \\
& \theta(t)=\theta_{0} \cos (\omega t)
\end{aligned}
$$




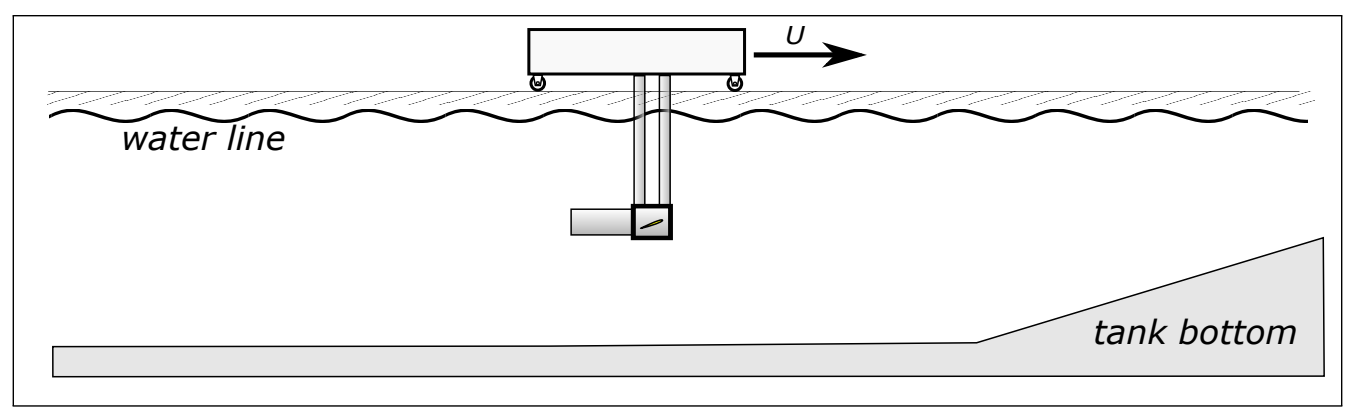

Figure 3.3.2: Side view cross section of the tow tank showing the depth profile created by adjustment of the tank's solid bottom panels. The foil drive is shown in the transition zone between freestream and ground effect test zone, and is shown mounted at a height which results in the closest bottom approach.[3]

Table 3.3.1: URI Experimental Parameters: Constants

\begin{tabular}{ccc}
\hline Carriage velocity & $U$ & $0.5 \frac{\mathrm{m}}{\mathrm{s}}$ \\
Roll amplitude & $\Phi_{0}$ & $12^{\circ}$ \\
Foil span length & $\mathrm{S}$ & $0.398 \mathrm{~m}$ \\
Foil chord length & $\mathrm{c}$ & $0.095 \mathrm{~m}$ \\
Foil planform area & $A_{p}=S c$ & $0.038 \mathrm{~m}^{2}$ \\
Foil aspect ratio & $A R=\frac{S}{c}$ & 4.2 \\
Distance to 70\% span & $r_{0.7}$ & $0.41 \mathrm{~m}$ \\
2-D heave of 70\% span point & $h_{0.7}$ & $0.17 \mathrm{~m}$ \\
Kinematic viscosity, $\mathrm{H}_{2} \mathrm{O}$ & $\nu$ & $10^{-6}$ \\
Reynolds number & $\frac{U c}{\nu}$ & 57,000 \\
\hline
\end{tabular}

Table 3.3.2: URI Experimental Parameters: Independent Variables

\begin{tabular}{lcc}
\hline Max. nominal angle of attack & $\alpha_{\max }$ & {$[20,25,30,35,40]^{\circ}$} \\
Strouhal number & $S t$ & {$[0.3,0.4,0.5]$} \\
Heave to chord ratio & $\frac{h_{0.7}}{c}$ & 1.8 \\
\hline
\end{tabular}

Table 3.3.3: URI Experimental Parameters: Dependent Variables

\begin{tabular}{ccc}
\hline Pitch amplitude & $\theta_{0}$ & chosen to obtain desired $\alpha_{0}$ \\
Oscillation frequency & $\omega$ & chosen to obtain desired $S t$ \\
\hline
\end{tabular}

where $\Phi_{0}$ is the amplitude of the roll motion, and $\theta_{0}$ is the amplitude of the pitch motion. $\Phi$ and $\theta$ are defined to be zero where the foil planform is parallel to the surface 


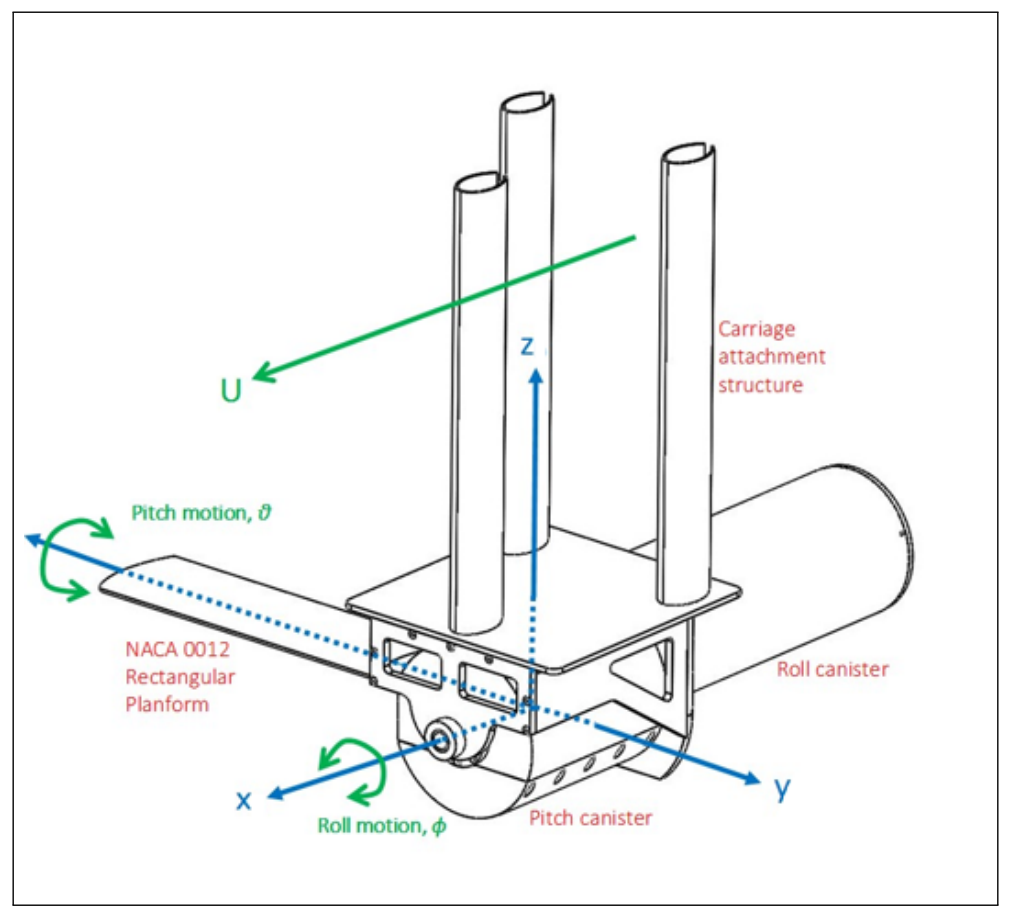

Figure 3.3.3: Foil drive mechanism and attachment. The submersible foil drive apparatus consists of two pressure housings, each containing a DC brush motor to actuate roll or pitch. Drive electronics are contained within the roll canister, while power and communication are supplied from the overhead carriage through underwater connectors. The foil shaft bearings are mounted to a rigid platform supported by a 6-DOF dynamometer inside the pitch canister, as detailed in Figure 3.3.5.[3]

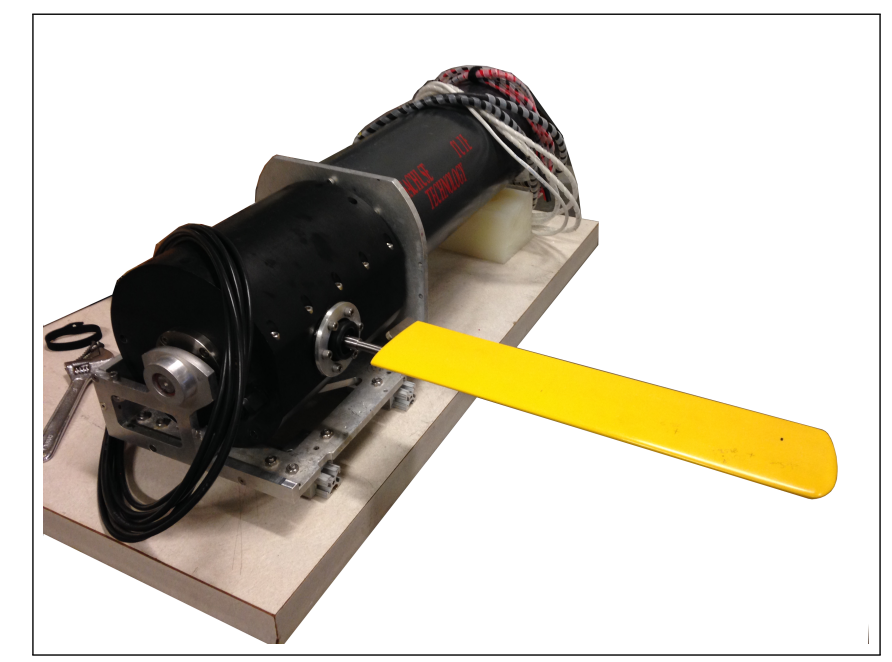

Figure 3.3.4: Image of foil drive apparatus prior to installation with foil mounted in place. The foil has a uniform NACA-0012 cross-section, with span of $0.398 \mathrm{~m}$ and chord of $0.095 \mathrm{~m}$, and is nominally rigid, with a titanium frame and cast outer shape.[3] 


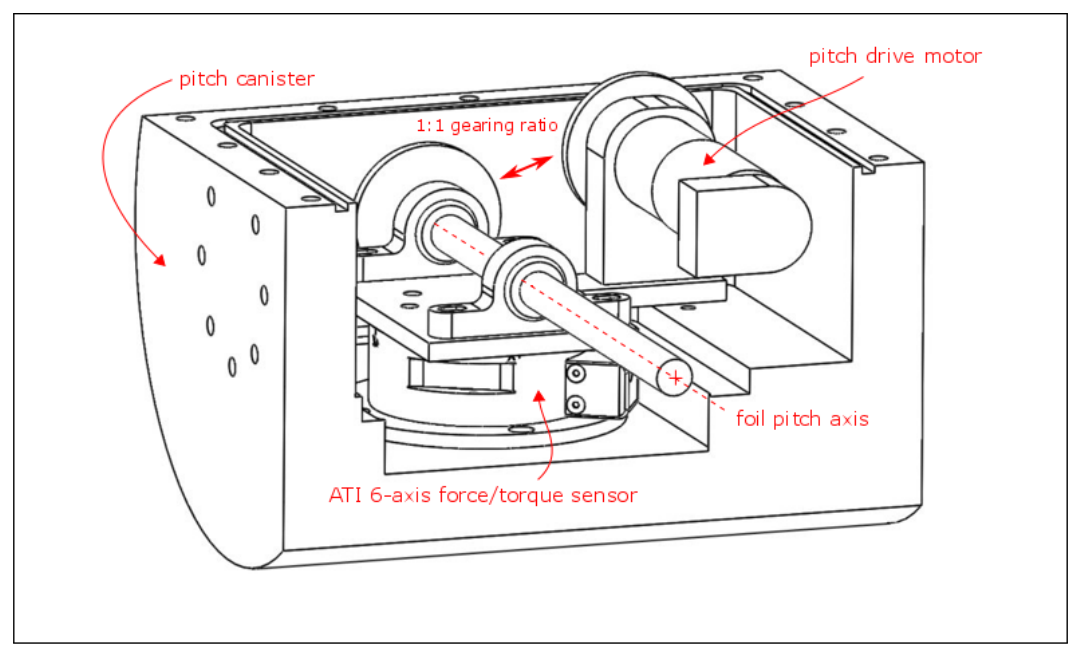

Figure 3.3.5: The pitch canister containing sensor and foil drive train. The front face of the canister is cut away in this view to show internal detail. The motor and shaft bearings are mounted to a rigid platform supported by the six-axis dynamometer. [3]

plane. The resulting nominal angle of attack as a function of time, $\alpha$, of the foil is given by,

$$
\alpha(t)=-\arctan \left(\frac{\omega r_{0.7} \Phi(t)}{U}\right)+\theta(t)
$$

\subsubsection{NUWC-Newport}

The oscillating foil device at NUWC-NPT is made up of two motors. The roll motor is a Maxon 355032 and the roll motor is a Maxon M081132 controlled by Maxon EPOS24/5 and EPOS24/1 control boards respectively. The motors, control boards, and amplifier are housed inside a $28.6 \mathrm{~cm}$ long, $10.6 \mathrm{~cm}$ diameter cylindrical waterproof shell. The roll and pitch shafts exit the canister in parallel and the pitch motion is rotated perpendicular to the roll through a bevel gear. The fin and canister was mounted to an AMTI UDW3-100 6-axis load cell connected to the test carriage as shown in Figure 3.3.6. The load cell has an MSA-6 signal conditioning box, measuring forces and moments with a range of $222 \mathrm{~N}$ in $\mathrm{X}$ and $\mathrm{Y}, 445 \mathrm{~N}$ in the $\mathrm{Z}$-axis, $11 \mathrm{Nm}$ moments in the $\mathrm{X}$ and $\mathrm{Y}$ directions, and $5.6 \mathrm{Nm}$ in $\mathrm{Z}$. The foil is oriented with the freestream velocity in the $\mathrm{X}$ axis, and the fin rolls and pitches about the $\mathrm{X}$ and $\mathrm{Z}$ axes respectively.

The onshore computer sends roll and pitch velocities to the motion control board to generate the oscillatory fin motion. The carriage to which the foil is attached travels the length of the tank where foil motion is stopped and then the carriage is returned to its 


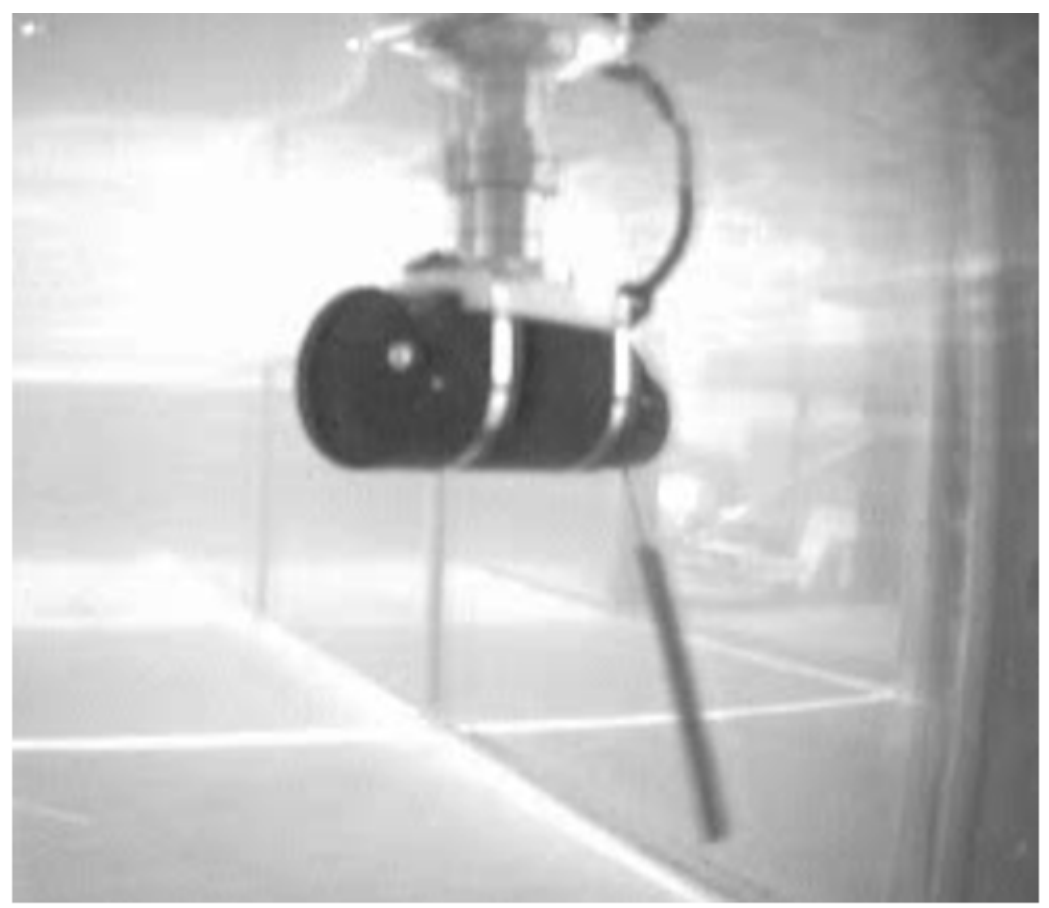

Figure 3.3.6: Photograph of the foil canister mounted to the force sensor inside the tank at NUWC-NPT

starting position. Instantaneous carriage velocity measurements are recorded alongside the force and motion data at a rate of $10 \mathrm{~Hz}$. Since the fin and motor canister are attached to the load cell, the drag of the canister and fin with zero angle of attack was recorded and removed from the force data in post processing.

The fin used at NUWC-NPT is a nominally rectangular planform foil with a NACA 0012-64 cross-section with the pitch axis located at the $\frac{1}{4}$ chord point. The foil span is $21.6 \mathrm{~cm}$ with a chord of $10.2 \mathrm{~cm}$. The distance from the roll axis to the start of the foils is $8.1 \mathrm{~cm}$ giving a $R_{0.7}$ of $25.3 \mathrm{~cm}$. The planform area of the foil is $219 \mathrm{~cm}^{2}$. These values are tabulated below in Table 3.3.4. Independent and dependent variables are also tabulated in Tables 3.3.5 and 3.3.6.

Table 3.3.4: NUWC-NPT Experimental Parameters: Constants

\begin{tabular}{ccc}
\hline Flow velocity & $U$ & $0.55 \frac{\mathrm{m}}{\mathrm{s}}$ \\
Roll amplitude & $\Phi_{0}$ & $40^{\circ}$ \\
Foil span length & $\mathrm{S}$ & $0.216 \mathrm{~m}$ \\
Foil chord length & $\mathrm{c}$ & $0.102 \mathrm{~m}$ \\
Foil planform area & $A_{p}=S c$ & $0.022 \mathrm{~m}^{2}$ \\
Foil aspect ratio & $A R=\frac{S}{c}$ & 2.125 \\
Distance to 70\% span & $r_{0.7}$ & $0.253 \mathrm{~m}$ \\
\hline
\end{tabular}


Table 3.3.4 - continued from previous page

2-D heave of $70 \%$ span point $\quad h_{0.7} \quad 0.325 m$

Kinematic viscosity, $\mathrm{H}_{2} \mathrm{O} \quad \nu \quad 10^{-6}$

Reynolds number $\quad \frac{U c}{\nu} \quad 51,000$

Table 3.3.5: NUWC-NPT Experimental Parameters: Independent Variables

\begin{tabular}{lcc}
\hline Pitch amplitude & $\theta_{0}$ & $35^{\circ}$ \\
Oscillation frequency & $f$ & $0.7 \mathrm{~Hz}$ \\
Heave to chord ratio & $\frac{h_{0.7}}{c}$ & 3.19 \\
Crossflow Angle & $\sigma$ & {$[-45,-30,0,30,45]$} \\
\hline
\end{tabular}

Table 3.3.6: NUWC-NPT Experimental Parameters: Dependent Variables

\begin{tabular}{ccc} 
Max. nominal angle of attack & $\alpha_{\max }$ & $14.5^{\circ}$ \\
Strouhal number & St & 0.37 \\
\hline
\end{tabular}

\subsubsection{3-D rolling and pitching foil model validation}

Force data from different 3-D oscillating foil experiments were used to validate the model. The code developed at URI was used to model experiments using both experimental setups. After examining the phase averaged lift and thrust signal of both experimental apparatuses the model was compared to mean lift and thrust coefficients of a foil over a range of kinematics. Finally, the model was used to predict the forces on a foil in the presence of a crossflow and compared to experimental data. The results of these comparisons are shown below.

\subsubsection{Phase averaged lift and thrust}

The model was used to predict the forces on the oscillating foil system at URI. The modeled results (black) are compared with data experimentally taken (red) in Figures 3.3.7a and 3.3.7b. They show the forces for a foil oscillating with $S t=.4$ and $\alpha_{\max }=30$ which is in the mid range of normal operating kinematics of this system. The model was able to predict the forces quite well with the exception of some small higher order harmonics in the lift signal and it slightly under predict the peak forces. The experimental forces plateau at their peaks where the modeled forces are more rounded. 


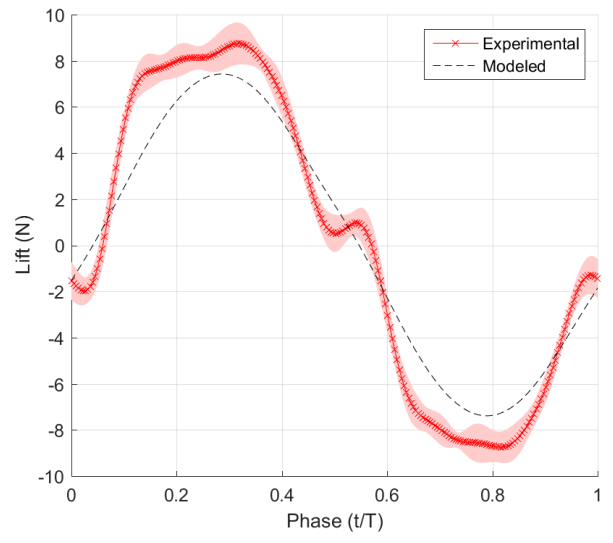

(a)

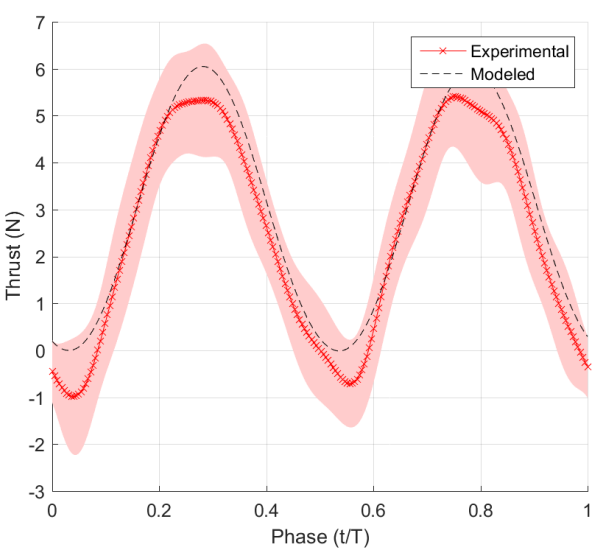

(b)

Figure 3.3.7: Phase averaged lift(a) and thrust(b) forces on a foil from URI with 2 standard deviations (red) compared to the modeled signal (black). $\left(S t=.4, \alpha_{\max }=30^{\circ}\right)$

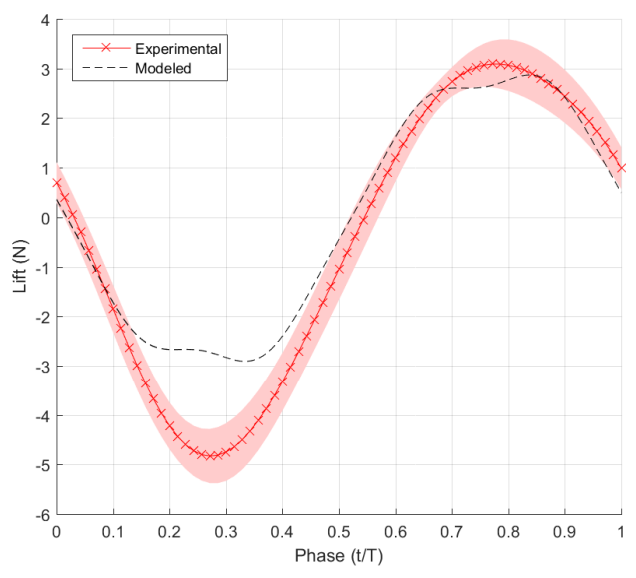

(a)

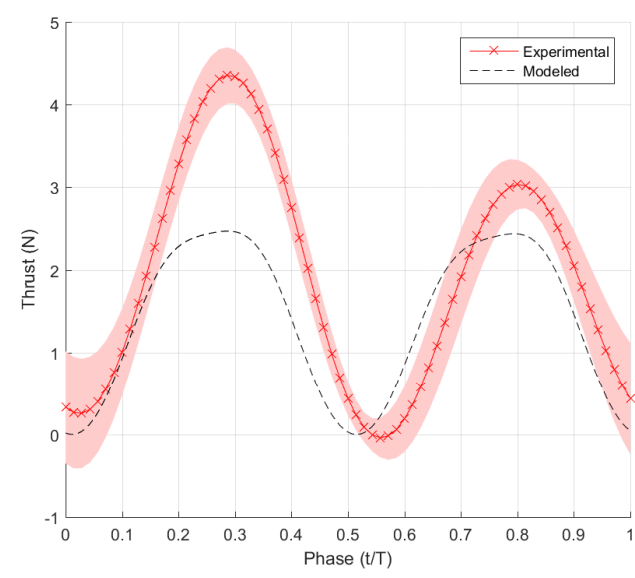

(b)

Figure 3.3.8: Phase averaged lift(a) and thrust(b) forces on a foil from NUWC-NPT with 2 standard deviations (red) compared to the modeled signal (black). $(S t=.37$, $\alpha_{\max }=14.5^{\circ}$ )

Next, the model was tested against the experimental apparatus at the NUWC-NPT. Figures 3.3.8a and 3.3.8b show the phase averaged experimental and modeled force signals for a typical flapping cycle of the NUWC-NPT system. The experimental signal also shows, shaded in red, two standard deviations on both sides of the mean. The modeled data has peaks that are flatter than that of the experimental data and the magnitude of one of the peaks is under predicted. It should also be noted that given symmetric sinusoidal motion, both peaks of the signals should be equal as is shown in the model but is not the case in the experimental signal. This could possibly be caused by slight asymmetries in the experimental setup. We can surmise that the model gives a good representation of the 
forces seen in both systems but there are still some higher order hydrodynamic effects that do not appear in the model.

\subsubsection{Range of Kinematics}

Since the model could predict the forces on different types of foils in normal operating conditions, the scope was expanded to include a foil flapping at different kinematics. The model was compared to experiments performed with the URI flapping foil system where the forces on the foil were recorded over a range of Strouhal numbers from 0.3 to 0.5 and maximum angles of attack from $20^{\circ}$ to $40^{\circ}$. The results are reported in mean lift and thrust coefficients as defined in Section 3.1.4 since these will be most of interest in the control system described later in Section 3.4.

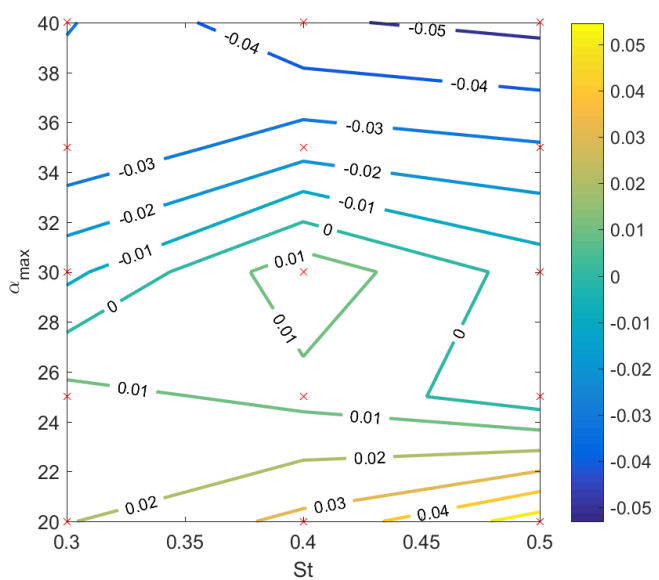

(a)

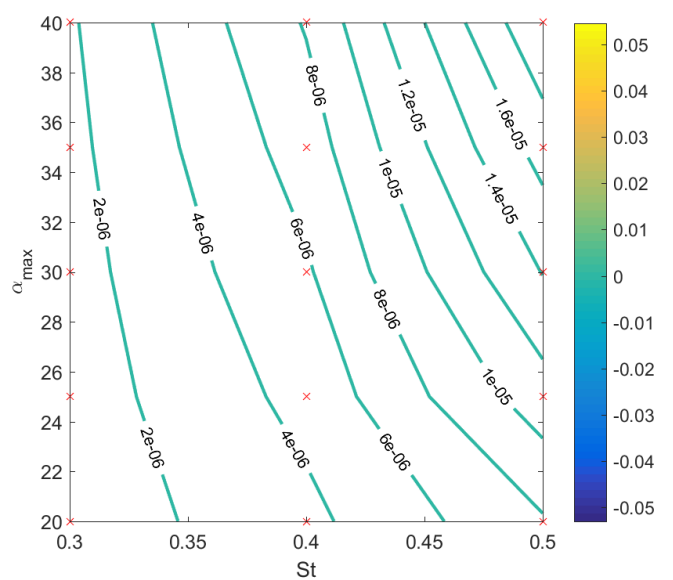

(b)

Figure 3.3.9: Mean lift coefficients across all tested kinematics (a) compared to modeled results (b)

Contours of mean lift coefficient from experimental and modeled force data are shown in Figures $3.3 .9 \mathrm{a}$ and $3.3 .9 \mathrm{~b}$ respectively. The model shows the mean lift coefficient across the entire range of kinematics to be practically zero. The experimental data has non zero mean lift coefficients dependent mostly on Strouhal number. This is likely caused by asymmetry in the experimental system at URI. The contours of mean thrust coefficient are shown in Figure 3.3.10. The model overestimates the thrust force across the entire range of kinematics and does not capture the decrease in thrust seen as the experimental kinematics approach the higher maximum angles of attack.

The difference in mean lift and thrust coefficients across all the kinematics tested are 


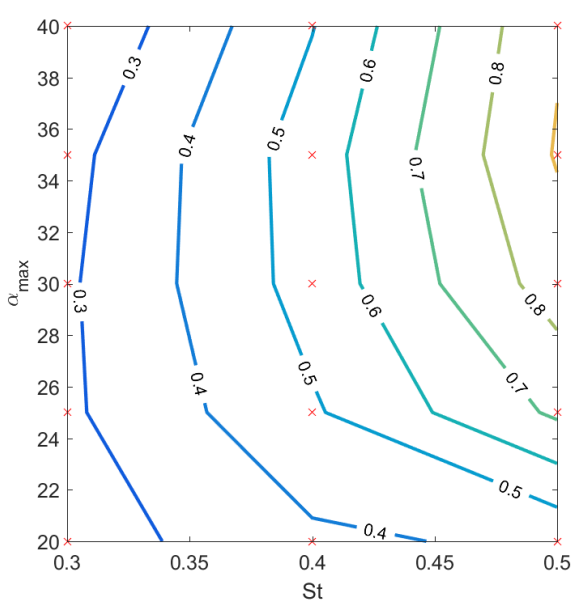

(a)

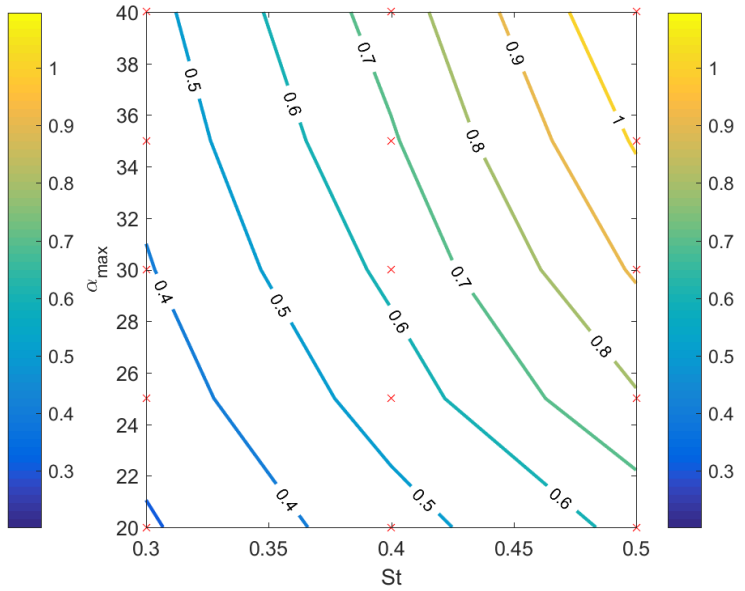

(b)

Figure 3.3.10: Mean thrust coefficients across all tested kinematics(a) compared to modeled results(b)

shown in Figures 3.3.11a and 3.3.11b respectively. The modeled mean lift coefficient has a maximum of approximately 0.05 at the highest Strouhal number and both high and low maximum angles of attack with the model over estimating at high angles of attack and under estimating at high angles of attack. Since the modeled data had zero mean lift coefficient across the entire range of data, the error comes mostly from the experimental data being nonzero and the shape of the difference contours is the same as the mean lift coefficient contours of the experimental data. In Figure 3.3.11b it can be seen that the model overestimates the mean thrust coefficient with a maximum error of approximately 0.25. The model appears to perform best at low strouhal and low angles of attack and worsens as maximum angles of attack or strouhal number increases.

Figures 3.3.12a and 3.3.12b show the percent difference in the amplitudes of the lift and thrust signals between experimental and modeled data. Difference in the prediction of lift amplitudes doesn't vary much with changes in kinematics and ranges between 20 and 30\% error. The amplitudes of the thrust signals, however, can vary by up to 40 percent at low maximum angles of attack but can reach as low as zero at higher maximum angles of attack but Strouhal number has little effect on the accuracy of the thrust amplitude prediction.

\subsubsection{Cross Flow}

After validating the model over a range of kinematics, crossflow and non zero pitch biases were introduced into the system. The following data sets were taken using the NUWC- 


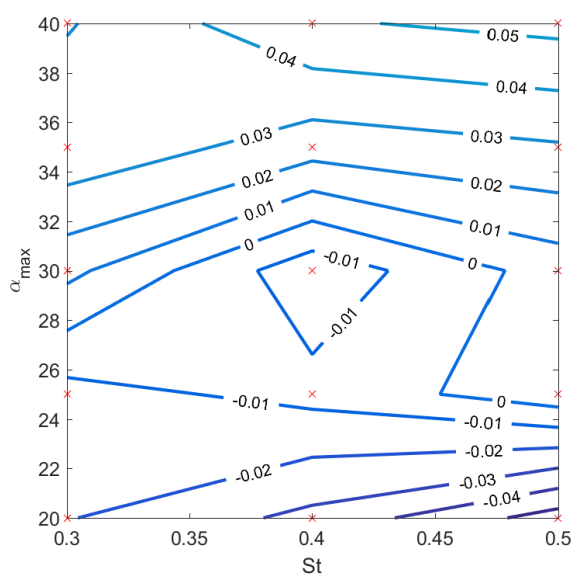

(a)
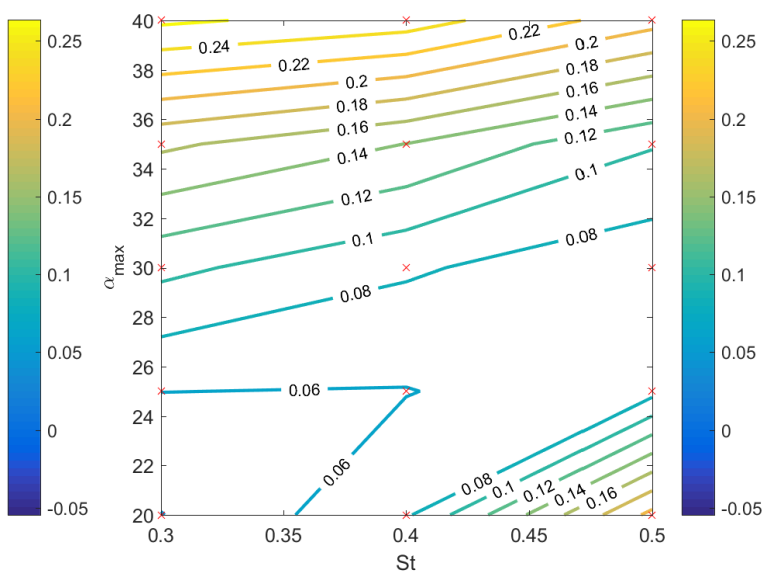

(b)

Figure 3.3.11: Difference in mean coefficients between experimental and modeled. (a) Mean Lift (b) Mean Thrust

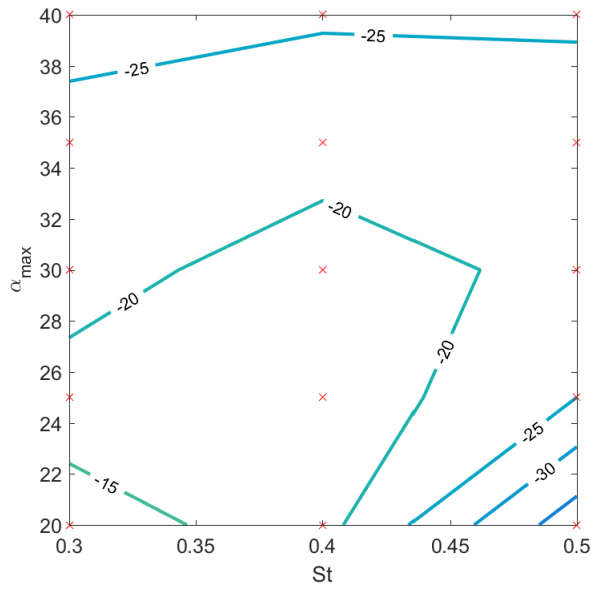

(a)

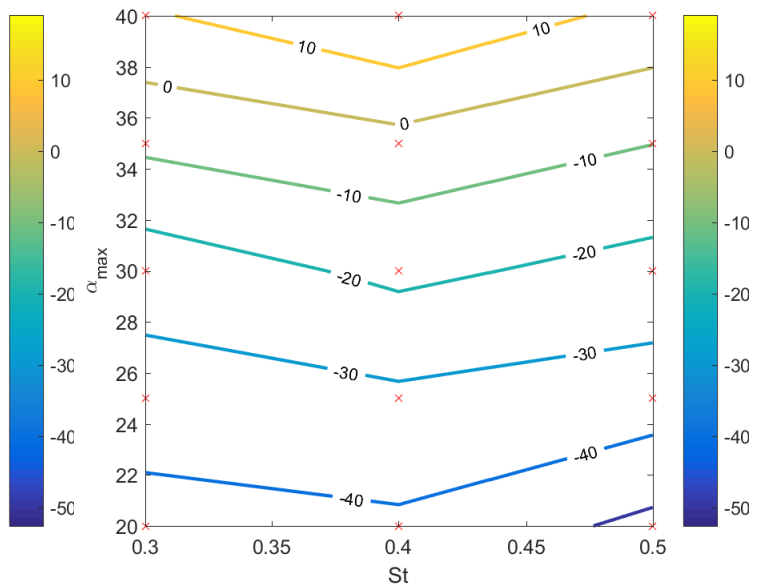

(b)

Figure 3.3.12: Percent[\%] difference in signal amplitudes between experimental and modeled. (a) Lift (b) Thrust

NPT flapping foil system over a range of crossflows and pitch biases but only one flapping kinematic. Figure 3.3.13 shows the phase averaged force signals of three different crossflow angles with no pitch bias in order to observe the effect of crossflow on the lift and thrust forces. Seen in Figures 3.3.13a and 3.3.13b, the crossflow creates asymmetry in the force signal. The downstroke has now shifted to a higher relative velocity while the upstroke has a slower relative velocity, or visa versa. This shift in relative velocity makes one stroke produce stronger forces than the other. The model shows the same effect and accurately models the shape of the force signal in the presence of a crossflow. 


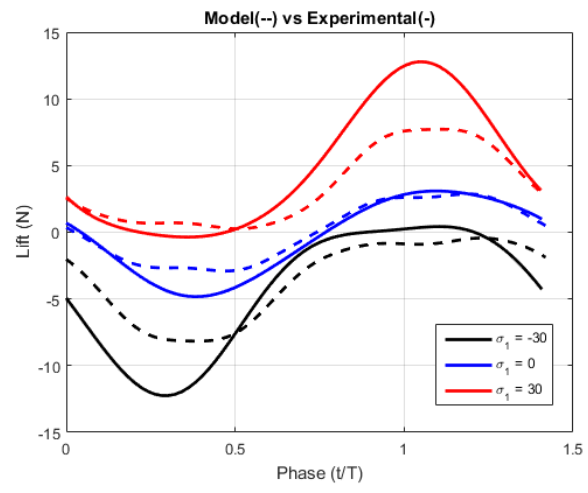

(a)

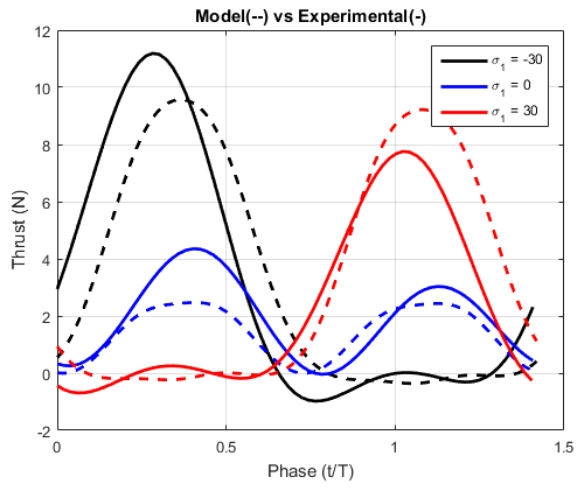

(b)

Figure 3.3.13: Modeled versus Experimental phased averaged lift force(a) and thrust force(b) for 3 different crossflow cases.

In order to counteract crossflow, a pitch bias is often added to the oscillatory motion to achieve a zero mean lift coefficient. Therefore, the model was tested against experimental force data of the foil flapping in the presence of different angles of crossflow with non zero pitch biases applied to the flapping motion. It should be noted that the angle of the crossflow and angle of pitch bias are opposed so that a $-30^{\circ}$ pitch bias would orient a foil directly into a $30^{\circ}$ crossflow. In Figure 3.3.14 the mean lift and thrust coefficients of the experimental and modeled data sets are presented for a given pitch bias. The angle of the crossflow in each test is denoted by the color of the data points. In Figure 3.3.14a the model accurately predicts the mean lift coefficient for different combinations of crossflow and pitch bias. However, seen in Figure 3.3.14b the accuracy of the mean thrust coefficients decays as the crossflow and pitch bias angles diverge. The greatest error is seen when the foil pitch is biased in the direction away from the crossflow. 


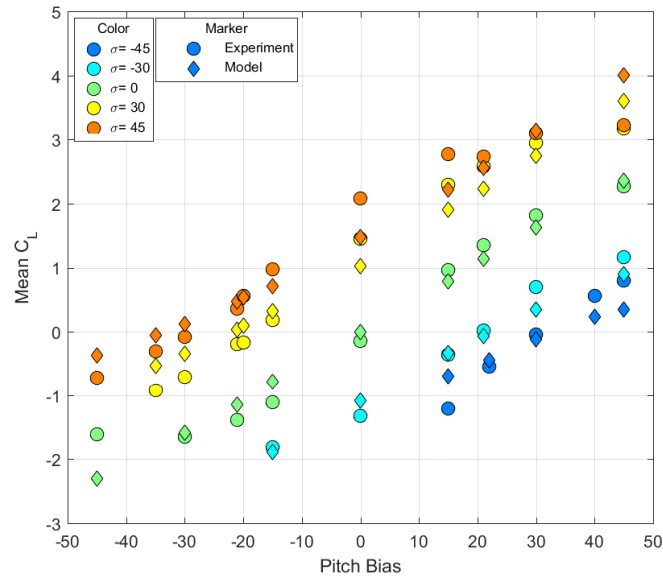

(a)

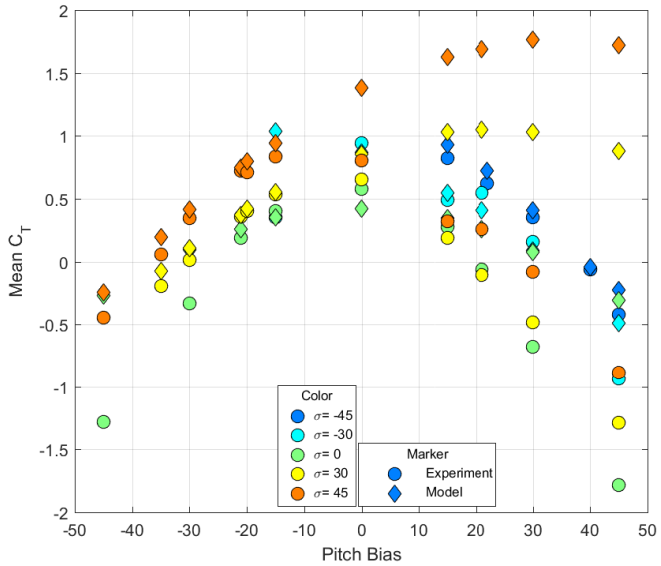

(b)

Figure 3.3.14: Experimental vs modeled mean lift coefficients(a) and mean thrust coefficients(b) for different crossflow and pitch bias angles

\subsection{Using Analytic Model as a Design Tool}

This model of the forces on a foil undergoing oscillatory motion is a potentially powerful tool for vehicles using this form of propulsion. We propose to use this model to develop a control system for a rolling and pitching foil that will compensate for and estimate a crossflow. During normal cruising operations, a vehicle maintains a desired mean thrust coefficient and a zero mean lift coefficient. Using feedback from the embedded force sensor we aim to control the vehicle to obtain desired force coefficients. Given a desired mean thrust coefficient of $\overline{C_{T}}$ des $=0.5$ the control system will adjust the flapping frequency and pitch bias in order to obtain the desired thrust coefficient while driving the mean lift coefficient to zero. The pitch amplitude will be calculated to achieve a maximum angle of attack for the current Strouhal number assuming a zero crossflow. It is understood that in the presence of crossflow the maximum angle of attack will vary with crossflow, but this method is employed to simplify and standardize the calculation of the pitch amplitude for a given Strouhal number.

\subsubsection{System Model}

The states in the system to be controlled are chosen to be the error in the mean lift and thrust coefficients as defined in (3.17). The state space system matrices are also defined 
below.

$$
\begin{aligned}
& {\overline{C_{T}}}_{\text {des }}=0.5 \\
& {\overline{C_{L}}}_{\text {des }}=0 \\
& E_{T}=\overline{C_{T}} \text { des }-\overline{C_{T}} \\
& E_{L}={\overline{C_{L}}}_{d e s}-{\overline{C_{L}}} \\
& x=\left[\begin{array}{l}
E_{T} \\
E_{L}
\end{array}\right] \quad u=\left[\begin{array}{l}
\delta S t \\
\delta \theta_{B}
\end{array}\right] \\
& \mathbf{A}=\left[\begin{array}{ll}
0 & 0 \\
0 & 0
\end{array}\right] \quad \mathbf{B}=\left[\begin{array}{ll}
\frac{\partial C_{T}}{\partial S_{t}}\left(S t, \alpha_{\max }, \theta_{B}\right) & \frac{\partial C_{T}}{\partial \theta_{B}}\left(S t, \alpha_{\max }, \theta_{B}\right) \\
\frac{\partial C_{L}}{\partial S t}\left(S t, \alpha_{\max }, \theta_{B}\right) & \frac{\partial C_{L}}{\partial \theta_{B}}\left(S t, \alpha_{\max }, \theta_{B}\right)
\end{array}\right] \\
& \mathbf{C}=\left[\begin{array}{ll}
1 & 0 \\
0 & 1
\end{array}\right] \quad \mathbf{D}=\left[\begin{array}{ll}
0 & 0 \\
0 & 0
\end{array}\right]
\end{aligned}
$$

The controllability matrix was formed for each continuous system created across the entire range of operating parameters and each was verified that it was full rank. Furthermore, since there are no complex poles of the system, the discrete time systems are controllable for all sampling intervals. Since each is controllable, the feedback loop was closed creating the system in (3.21) where $L$ is the feedback gain matrix.

$$
\left[\begin{array}{c}
E_{T}[k+1] \\
E_{L}[k+1]
\end{array}\right]=\left[\begin{array}{cc}
1-L_{(1,1)} \frac{\partial C_{T}}{\partial S t}\left(S t, \alpha_{\max }, \theta_{B}\right) & -L_{(1,2)} \frac{\partial C_{T}}{\partial \theta_{B}}\left(S t, \alpha_{\max }, \theta_{B}\right) \\
-L_{(2,1)} \frac{\partial C_{L}}{\partial S t}\left(S t, \alpha_{\max }, \theta_{B}\right) & 1-L_{(2,2)} \frac{\partial C_{L}}{\partial \theta_{B}}\left(S t, \alpha_{\max }, \theta_{B}\right)
\end{array}\right]\left[\begin{array}{c}
E_{T}[k] \\
E_{L}[k]
\end{array}\right]
$$

\subsubsection{Gain Calculation and Simulation}

With access to the working flapping foil force model, it was possible to pre-calculate the feedback gains for a range of operating conditions. Separate gains were found for a range of Strouhal numbers, maximum angles of attack, and pitch bias angles by calculating the gradients of mean lift and thrust coefficients provided by the analytical force model to create a system model for each operating point and using a pole placement method to calculate the feedback gain. These gains were stored so that during the control tests, the 
correct gain could be quickly obtained for the current operating conditions of each step. The feedback gains were calculated to place the poles each closed looped system at the roots of a 2nd order Bessel polynomial normalized by the desired settling time. The desired settling time was 3 times the flapping period derived from each Strouhal number. With the gains calculated, the control system could be simulated by feeding the control inputs back into the analytical model code. The results are shown in Figures 3.4.1 and 3.4.2.

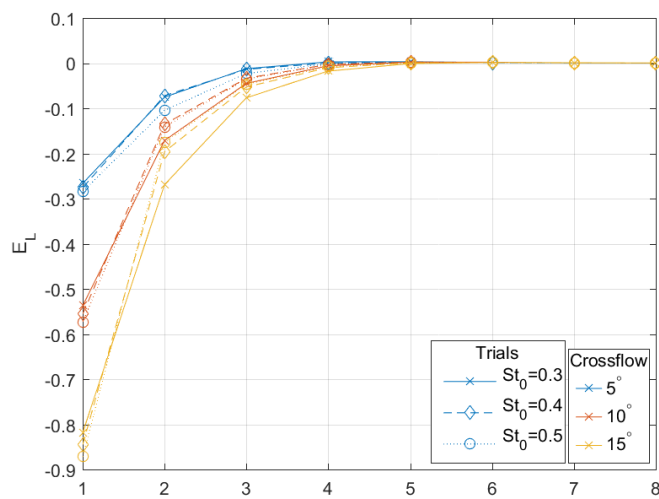

(a)

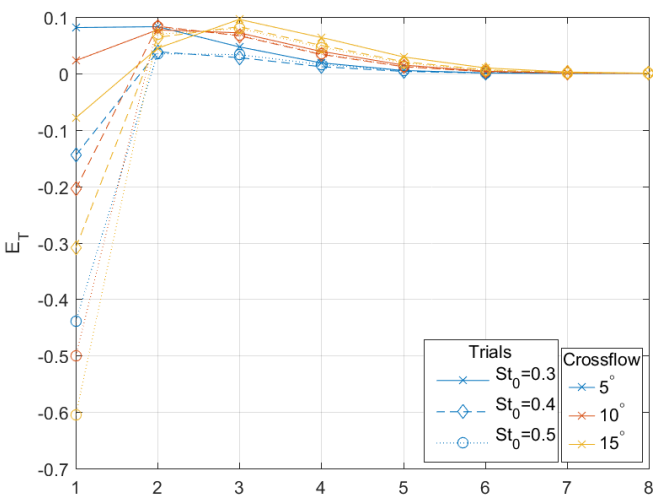

(b)

Figure 3.4.1: Simulation of error in both the mean lift(a) and thrust(b) coefficients over time. Each crossflow angle is designated by a different color and each initial condition corresponds to a different line style.

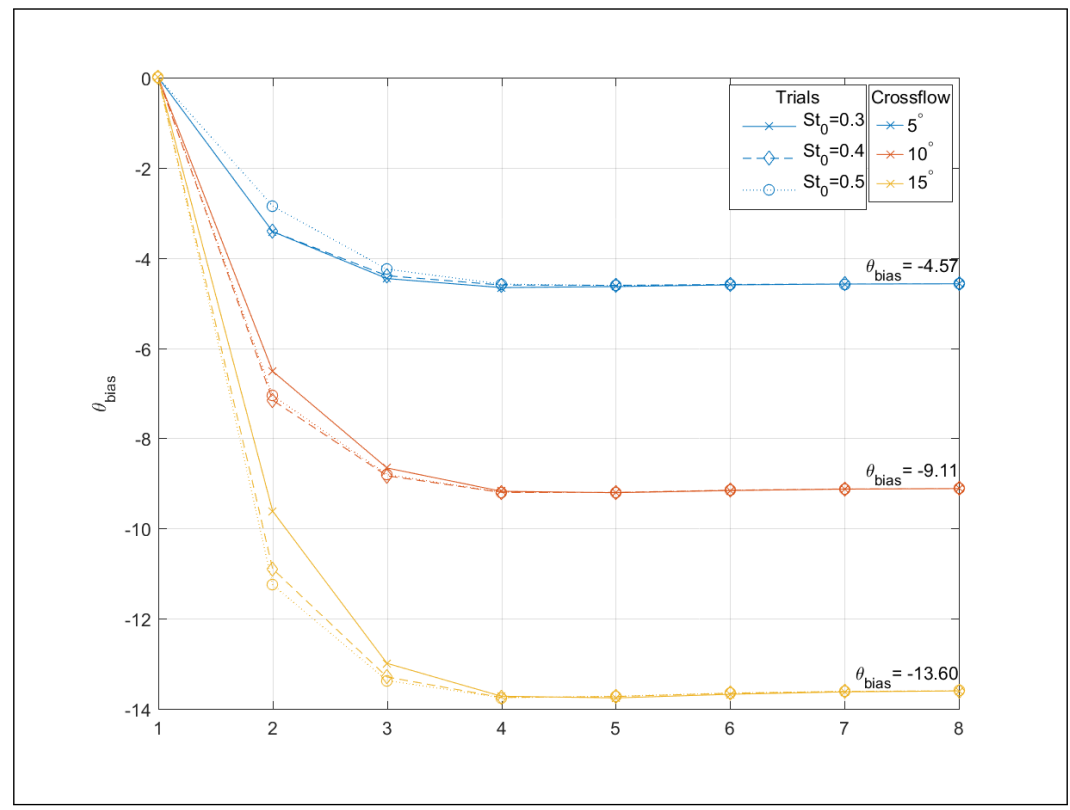

Figure 3.4.2: Simulation of the pitch bias of the system over time. Each crossflow angle is designated by a different color and each initial condition corresponds to a different line style. 
Figures 3.4.1a and 3.4.1b show the error in mean lift and thrust coefficients, $E_{L}$ and $E_{T}$, for each step of the simulation and Figure 3.4.2 shows the evolution of the pitch bias angle, $\theta_{\text {bias }}$. Each crossflow angle is designated by a different color in the figures and each initial condition corresponds to a different line style. The initial conditions correspond with initial errors ranging from -0.9 to -0.3 and -0.6 to 0.1 for lift and thrust respectively. For each case the errors decay to zero in about 5 time steps. The pitch bias in Figure 3.4.2 settles to the values $\left[-4.57^{\circ},-9.11^{\circ},-13.60^{\circ}\right]$ for crossflow angles of $\left[5^{\circ}, 10^{\circ}, 15^{\circ}\right]$ respectively. The lift coefficient settles to zero when the pitch bias angle is approximately $91 \%$ of the crossflow angle. This relationship will allow for an improved estimation of the crossflow angle when supplied with experimental data.

\subsubsection{Crossflow Compensation using force feedback}

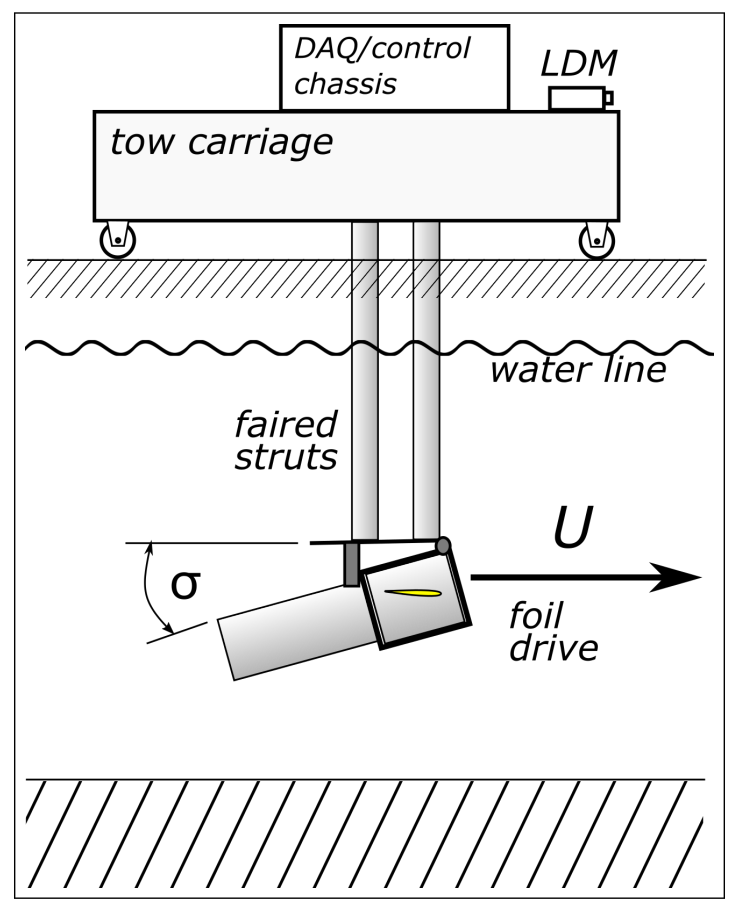

Figure 3.4.3: Modified URI experimental apparatus to allow for crossflow testing.

In order to test the control system designed, the experimental apparatus at URI was modified to allow for the application of a crossflow by adding a hinge to the assembly, shown in Figure 3.4.3, enabling the foil canisters to be rotated an angle $\sigma$ with respect the oncoming flow. Each experimental trial followed these prescribed steps:

1. The foil was run at an initial set of kinematics 
2. The force data recorded was processed to determine the current mean lift and thrust coefficients and the error of each.

3. Using the error and the pre-calculated feedback gains, a change in $S t$ and $\theta_{\text {bias }}$ were found.

4. The foil was run with the new kinematics and this cycle continued until the values settled at $\overline{C_{T}}$ des and $\bar{C}_{L}$ des

Tests were run for each crossflow angle $\sigma \approx[5,10,15]$, starting at 3 different initial Strouhal numbers, $S t=[0.3,0.4,0.5]$, as listed in Table 3.4.1. For simplicity, the pitch amplitude of each set of kinematics was chosen to maintain a constant maximum angle of attack of $\alpha_{\max }=30$ assuming no presence of crossflow. The independent and dependent variables are shown in updated Tables 3.4.1 and 3.4.2.

Table 3.4.1: URI Crossflow Experimental Parameters: Independent Variables

\begin{tabular}{lcc}
\hline Max. nominal angle of attack & $\alpha_{\max }$ & $30^{\circ}$ \\
Initial Strouhal number & $S t_{0}$ & {$[0.3,0.4,0.5]$} \\
Initial Pitch bias & $\theta_{\text {bias } 0}$ & 0 \\
Heave to chord ratio & $\frac{h_{0.7}}{c}$ & 1.8 \\
Crossflow Angle & $\sigma$ & {$\left[5.25^{\circ}, 10^{\circ}, 14.43^{\circ}\right]$} \\
\hline
\end{tabular}

Table 3.4.2: URI Crossflow Experimental Parameters: Dependent Variables

\begin{tabular}{ccc}
\hline Pitch amplitude & $\theta_{0}$ & chosen to obtain desired $\alpha_{0}$ \\
Oscillation frequency & $\omega$ & chosen to obtain desired $S t_{0}$ \\
\hline
\end{tabular}

Figures 3.4.4 and 3.4.5 show the experimental results of the control system when tested on the flapping foil at URI. In each figure the cases are separated by color and line style which correspond to independent variables crossflow angle, $\sigma$, and initial Strouhal number, $S t_{0}$, respectively. There is greater overshoot in the experimental implementation of the control system than seen in simulation but the states settle in the same amount of time. The initial errors are also different than predicted from the model but this was expected due to the slight discrepancies seen between the analytical model and experiments described in Section 3.3.2. 


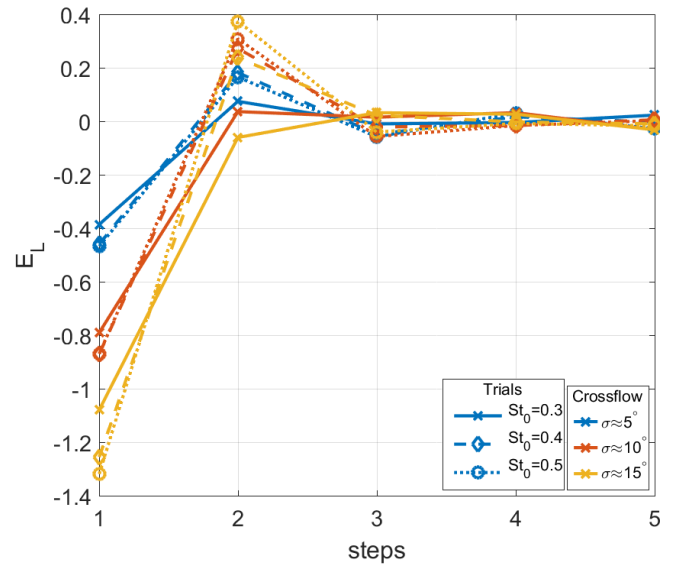

(a)

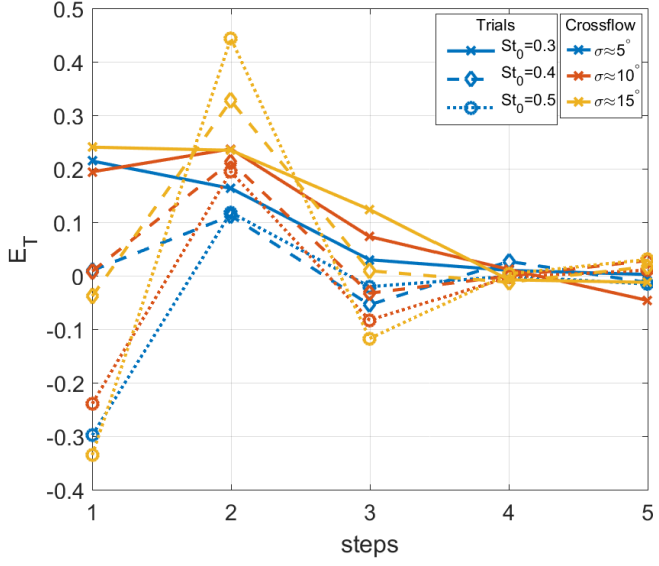

(b)

Figure 3.4.4: Experimental data showing the evolution of error in both the mean lift(a) and thrust(b) coefficients over time. Each crossflow angle is designated by a different color and each initial condition corresponds to a different line style.

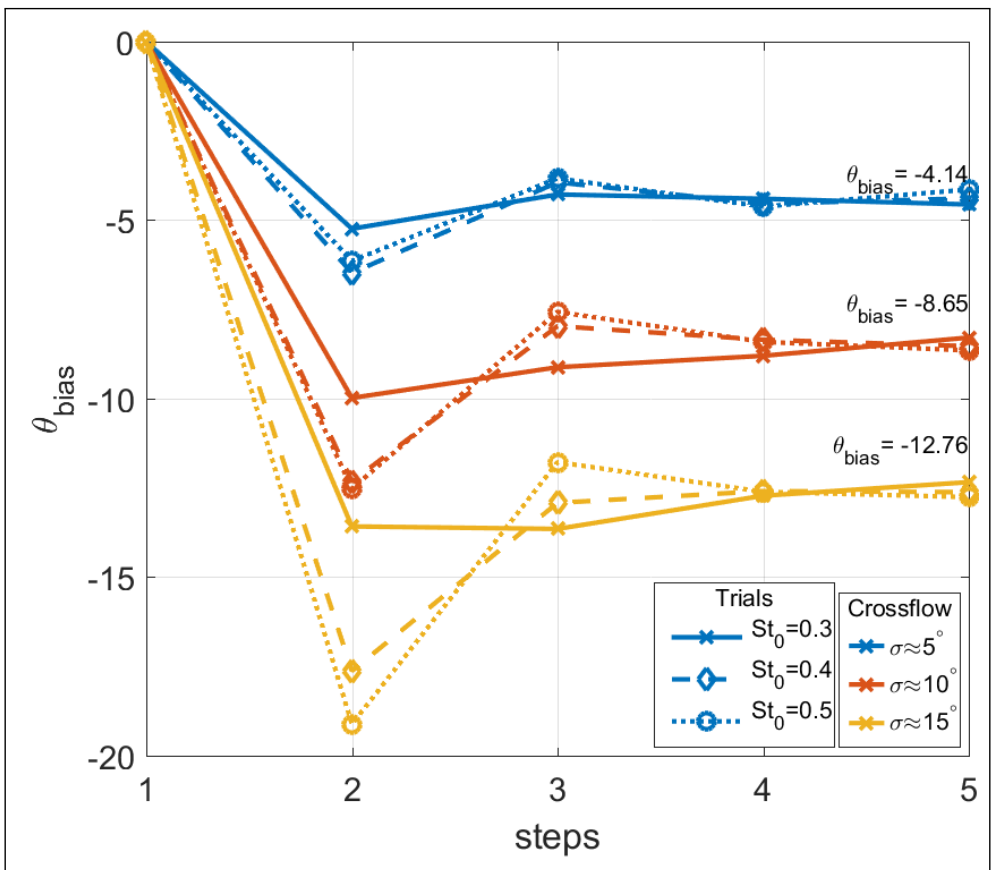

Figure 3.4.5: Experimental data showing the evolution of pitch bias angle over time. Each crossflow angle is designated by a different color and each initial condition corresponds to a different line style. 


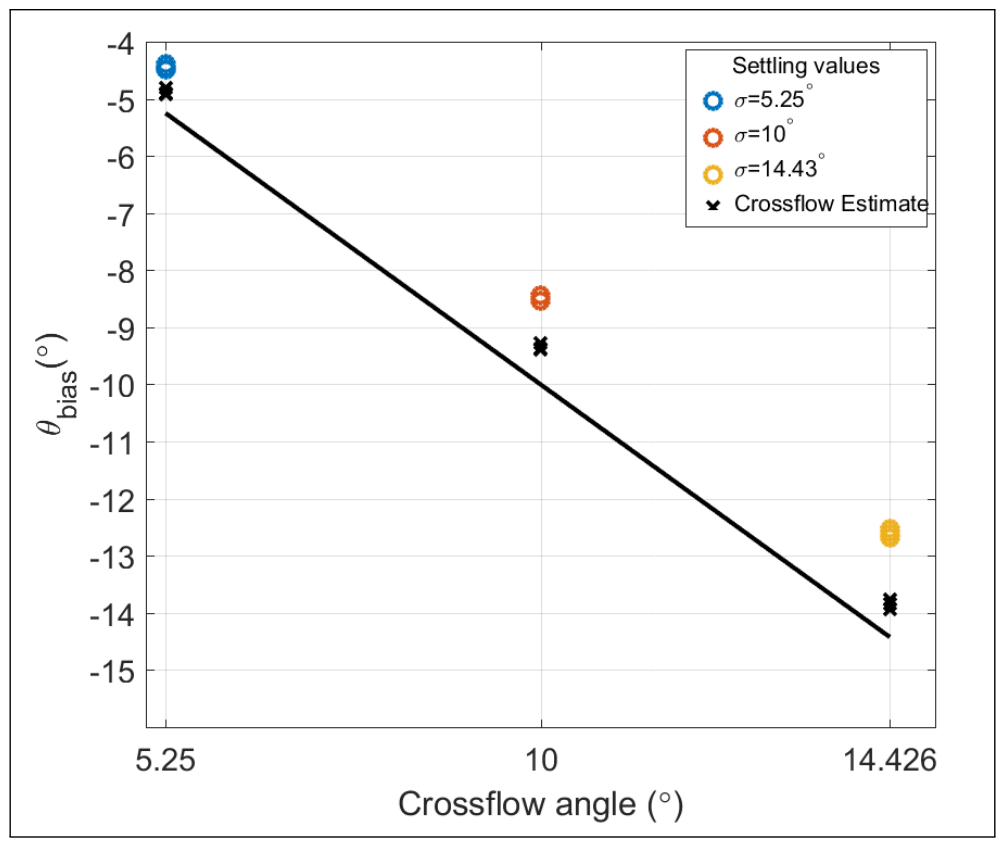

Figure 3.4.6: Final pitch bias angles compared to crossflow angle along with crossflow angle using the $91 \% \sigma$ relationship found during simulations.

Figure 3.4.6 shows the final pitch bias angle, $\theta_{\text {bias }}$, of each trial plotted against the crossflow angle of the trial. The reference lines $\theta_{\text {bias }}=\sigma$ is also plotted along side the experimental data. A mean lift coefficient of zero in a crossflow does not occur at a pitch bias angle equal and opposite of the crossflow. The pitch bias angle settles within approximately $1-1.5^{\circ}$ of the crossflow angle, however, using the $91 \%$ simulated relationship from the model the settling angles, the crossflow can be estimated to within approximately $0.5^{\circ}$.

\subsection{Discussion}

From all of the results shown here, we would like to highlight three distinct points:

- The code based on the analytical model provides accurate force prediction across a range of foil configurations, kinematics, and environmental factors.

- A simple control system combined with an analytical model can compensate for and estimate crossflow.

- This model is a valid design tool for designing control systems of 3-D rolling and pitching oscillating foils 


\subsubsection{The code based on the analytical model provides accurate force predic- tion across a range of foil configurations, kinematics, and environmental factors.}

We were able to encode the analytical model into a working MATLAB script that can be used to predict forces on oscillating foils. The results from our code matched those provided by [5]. Upon creating a working tool, we moved to test it against a new range of conditions. The model performed well predicting forces for both the foil at NUWC-NPT and the foil at URI. The prediction of the URI foil was slightly better possibly due to some suspect error in the experimental setup at NUWC-NPT since symmetric kinematics did not provide symmetric force signals. In Figure 3.3.8 it can be seen that the magnitude of one stroke of the flapping cycle is greater than the other. This is likely due to the device not being completely aligned with the flow. The model was tested against a range of kinematics and performed well with some slight over prediction of thrust at high $\alpha_{\max }$. The model was also able to accurately predict forces when a crossflow was added to the simulation. The model does start to fail as crossflow diverges too far from the pitch bias angle as seen in Figure 3.3.14. Overall, the code based on the analytical model is able to predict the forces on an oscillating foil for variety of cases within normal operating conditions of an oscillating foil. It should be noted that the model was able to accurately predict the forces without any sort of tuning parameters and was able to be implemented for different systems by simply knowing the geometry and motion profiles of the foils.

\subsubsection{A simple control system combined with an analytical model can compen- sate for and estimate crossflow.}

The state feedback regulator designed in this paper was able to compensate for increasing angles of crossflow and was able to achieve the desired mean lift and thrust coefficients. There is some overshoot especially when correcting for large angles of crossflow but still settles to the desired values. In this system both the lift and thrust are coupled, but it seems that the error in mean thrust which is more variable causes disturbances in the mean lift coefficient. This could be fixed by decoupling the two, where the change in Strouhal number is based solely on the error in mean thrust and the pitch bias angle is based solely on the error in mean lift. From the analytical model we were able to simulate how the control system would perform and also found that a zero mean lift coefficient would not occur when the pitch bias oriented the fin motion directly into the flow but at an angle 
approximately $91 \%$ of the crossflow angle. This is most likely due to the fact that while the pitch can be aligned with the flow, the roll motion is still constrained to be perpendicular to the canisters. This means that as the crossflow value increases the roll motion which is normally completely perpendicular to the flow has an increasing amount of motion inline with the flow which will affect the angle of attack of the foil through out its motion. More information on the effect of adding inline motion to oscillating foils can be found in [8]. The simulation was able to provide the $91 \% \sigma$ relationship that allowed for estimation of the crossflow angle to within half of a degree by dividing the pitch bias angle of zero mean lift by the $91 \%$. This simple system would allow a vehicle to maintain course, rejecting the steady disturbance of a crossflow, while simultaneously estimating information about the surrounding environment.

\subsubsection{This model is a valid design tool for designing control systems of 3-D rolling and pitching oscillating foils}

The analytical model accurately predicts the forces on a foil and has been shown to perform well predicting lift and thurst forces under different operating conditions and in the presence of constant disturbances such as a crossflow. This paper describes a simple state feedback regulator that was designed and simulated using the model. The control system performed remarkably similar to simulated results and was able to compensate for a crossflow on the body. The model was also able to provide information for more accurate estimation of the crossflow angle. Being able to use the model to rapidly design and test control systems is a powerful tool and can be significantly more cost efficient than running full scale vehicle experiments. The model proved to be a useful tool for designing a control system and was able to accurately predict the performance of a control system without any need of tuning.

\subsection{Conclusion}

As vehicles with oscillating foil propulsion progress, their control systems will also need to perform in more difficult conditions. Having force feedback implemented on individual foils will allow for more complex vehicle maneuvers. Individual foil control can improve the agility of a vehicle allowing for maneuvers such as corkscrews, and barrel rolls. Station keeping and low-speed hovering or investigative maneuvers would also improve with better foil control. New tools for control system design will be needed to enable cheaper more 
efficient research into these areas. A code was developed to replicate the analytical model published in [5]. This code was validated against results in [5] and was also validated against experimental data of various foil configurations, kinematic parameters, and environmental conditions. The code was able to replicate previously published results, accurately predict forces on oscillating foil configurations at NUWC-NPT and URI, predict mean force coefficients over a range of foil kinematics and accurately estimate the effect that crossflow will have on the forces experienced by an oscillating foil. Once the code was validated, it was used to design and simulate a simple control system. The control system was implemented on the foil system at URI and was able to compensate and coupled with information from the model, estimate the crossflow angle within a half of a degree.

\subsection{Future Work}

The steps taken here open up various paths for further research. The code can be used to test the model against more complicated environmental conditions such as unsteady disturbances such as the presence of wave induced velocities. The control in this paper was done in discrete tests off-line post processing before new kinematics being input to the foil system. Modifications could be made to the oscillating foil system to allow for real time control. The control in this paper was done with mean lift and thrust coefficients, the model can output instantaneous lift forces which could be used for more complex motion control of the foil, including non-sinusoidal oscillating motions. In this paper we used a simple feedback regulator to drive the error of the system to zero. More complicated control systems could be simulated to improve robustness and response characteristics.

\section{List of References}

[1] S. C. Licht, "Biomimetic oscillating foil propulsion to enhance underwater vehicle agility and maneuverability," Ph.D. dissertation, Massachusetts Institute of Technology and Woods Hole Oceanographic Institution, 2008.

[2] D. Beal, H. Leinhos, A. Fredette, and R. Berube, "Unified Scaling for Flapping Fins," IEEE Journal of Oceanic Engineering, vol. 38, no. 1, pp. 1-11, 2013.

[3] M. Perkins, D. Elles, G. Badlissi, A. Mivehchi, J. Dahl, and S. Licht, "Rolling and pitching oscillating foil propulsion in ground effect," Bioinspiration $\&$ biomimetics, vol. 13, no. 1, p. 016003, 2017.

[4] R. J. Vaccaro, Digital control: a state-space approach. McGraw-Hill New York, 1995, vol. 196. 
[5] J. S. Izraelevitz, Q. Zhu, and M. S. Triantafyllou, "State-space adaptation of unsteady lifting line theory: twisting/flapping wings of finite span," AIAA Journal, pp. 1279-1294, 2017.

[6] R. T. Jones, "The unsteady lift of a wing of finite aspect ratio," 1940.

[7] V. Polidoro, "Flapping foil propulsion for cruising and hovering autonomous underwater vehicles," Master's thesis, Massachusetts Institute of Technology, 2003.

[8] J. S. Izraelevitz and M. S. Triantafyllou, "Adding in-line motion and model-based optimization offers exceptional force control authority in flapping foils," Journal of Fluid Mechanics, vol. 742, pp. 5-34, Mar. 2014. 


\section{BIBLIOGRAPHY}

Abramowski, T., "Numerical investigation of airfoil in ground proximity," Journal of Theoretical and Applied Mechanics, vol. 45, pp. 425-436, 2007.

Ahmed, M. and Sharma, S., "An investigation on the aerodynamics of a symmetrical airfoil in ground effect," Experimental Thermal and Fluid Science, vol. 29, no. 6, pp. 633-647, July 2005.

Anderson, J. M., Streitlien, K., Barrett, D. S., and Triantafyllou, M. S., "Oscillating foils of high propulsive efficiency," Journal of Fluid Mechanics, vol. 360, pp. 41-72, Apr. 1998.

Anderson, J. M. and Chhabra, N. K., "Maneuvering and Stability Performance of a Robotic Tuna," Integrative and Comparative Biology, vol. 42, no. 1, pp. 118-126, Feb. 2002.

Beal, D., Leinhos, H., Fredette, A., and Berube, R., "Unified Scaling for Flapping Fins," IEEE Journal of Oceanic Engineering, vol. 38, no. 1, pp. 1-11, 2013.

Blevins, E. and Lauder, G. V., "Swimming near the substrate: a simple robotic model of stingray locomotion," Bioinspiration $\mathscr{E}$ Biomimetics, vol. 8, no. 1, p. 016005, Mar. 2013.

Blondeaux, P., Fornarelli, F., Guglielmini, L., Triantafyllou, M. S., and Verzicco, R., "Numerical experiments on flapping foils mimicking fish-like locomotion," Physics of Fluids, vol. 17, no. 11, p. 113601, Nov. 2005.

Buchholz, J. H. J. and Smits, A. J., "On the evolution of the wake structure produced by a low-aspect-ratio pitching panel," Journal of Fluid Mechanics, vol. 546, no. -1, p. 433, Dec. 2005.

Cui, E. and Zhang, X., "Ground effect aerodynamics," Encyclopedia of Aerospace Engineering, vol. 1, no. Part 3, pp. 245-256, 2010.

Dai, L., He, G., and Zhang, X., "Self-propulsion of a Flexible Plunging Foil Near a Solid Wall," Procedia Engineering, vol. 126, pp. 431-435, 2015.

Dai, L., He, G., and Zhang, X., "Self-propelled swimming of a flexible plunging foil near a solid wall," Bioinspiration \& Biomimetics, vol. 11, no. 4, p. 046005, July 2016.

Dean, R. G. and Dalrymple, R. A., Water wave mechanics for engineers and scientists. World Scientific Publishing Company, 1991, vol. 2.

Elles, D., "Analysis of Force Production by a Biologically Inspired Underwater Flapping Foil Near Solid Boundaries in Three Dimensional Flow," 2016.

Fernandez-Prats, R., Raspa, V., Thiria, B., Huera-Huarte, F., and Godoy-Diana, R., "Largeamplitude undulatory swimming near a wall," Bioinspiration 83 biomimetics, vol. 10, no. 1, p. 016003, 2015.

Filippas, E. and Belibassakis, K., "Hydrodynamic analysis of flapping-foil thrusters operating beneath the free surface and in waves," Engineering Analysis with Boundary Elements, vol. 41, pp. 47-59, Apr. 2014.

Flores, M. D., "Flapping motion of a three-dimensional foil for propulsion and maneuvering of underwater vehicles," Ph.D. dissertation, Massachusetts Institute of Technology, 2003. 
Georgiades, C., Nahon, M., and Buehler, M., "Simulation of an underwater hexapod robot," Ocean Engineering, vol. 36, no. 1, pp. 39-47, 2009.

Hoerner, S. F. and Borst, H. V., "Fluid-dynamic lift: practical information on aerodynamic and hydrodynamic lift," 1985.

Izraelevitz, J. S. and Triantafyllou, M. S., "Adding in-line motion and model-based optimization offers exceptional force control authority in flapping foils," Journal of Fluid Mechanics, vol. 742, pp. 5-34, Mar. 2014.

Izraelevitz, J. S., Zhu, Q., and Triantafyllou, M. S., "State-space adaptation of unsteady lifting line theory: twisting/flapping wings of finite span," AIAA Journal, pp. 12791294, 2017.

Jones, K. D., Dohring, C. M., and Platzer, M. F., "Experimental and Computational Investigation of the Knoller-Betz Effect," AIAA Journal, vol. 36, no. 7, pp. 1240-1246, July 1998.

Jones, R. T., "The unsteady lift of a wing of finite aspect ratio," 1940.

Koochesfahani, M. M., "Vortical patterns in the wake of an oscillating airfoil," American Institute of Aeronautics and Astronautics, vol. 27, no. 9, pp. 1200-1205, Sept 1989.

Licht, S., Polidoro, V., Flores, M., Hover, F., and Triantafyllou, M., "Design and Projected Performance of a Flapping Foil AUV," IEEE Journal of Oceanic Engineering, vol. 29, no. 3, pp. 786-794, July 2004.

Licht, S. C., Wibawa, M. S., Hover, F. S., and Triantafyllou, M. S., "In-line motion causes high thrust and efficiency in flapping foils that use power downstroke," The Journal of experimental biology, vol. 213, no. 1, pp. 63-71, 2010.

Licht, S. C., "Biomimetic oscillating foil propulsion to enhance underwater vehicle agility and maneuverability," Ph.D. dissertation, Massachusetts Institute of Technology and Woods Hole Oceanographic Institution, 2008.

Long Jr, J. H., Schumacher, J., Livingston, N., and Kemp, M., "Four flippers or two? tetrapodal swimming with an aquatic robot," Bioinspiration 63 Biomimetics, vol. 1, no. 1 , p. $20,2006$.

Mivehchi, A., Dahl, J., and Licht, S., "Heaving and pitching oscillating foil propulsion in ground effect," Journal of Fluids and Structures, vol. 63, pp. 174-187, May 2016.

Perkins, M., Elles, D., Badlissi, G., Mivehchi, A., Dahl, J., and Licht, S., "Rolling and pitching oscillating foil propulsion in ground effect," Bioinspiration 8 biomimetics, vol. 13, no. 1, p. 016003, 2017.

Polidoro, V., "Flapping foil propulsion for cruising and hovering autonomous underwater vehicles," Master's thesis, Massachusetts Institute of Technology, 2003.

Prempraneerach, P., Hover, F. S., and Triantafyllou, M. S., "The effect of chordwise flexibility on the thrust and efficiency of a flapping foil," Proceedings Unmanned, Untethered Submersible Technology, 2003.

Quinn, D. B. and Smits, A. J., "Rectangular panels undergoing pitch oscillation in ground effect," in The 1000 Island Fluids Meeting, 2012.

Quinn, D. B., Lauder, G. V., and Smits, A. J., "Flexible propulsors in ground effect," Bioinspiration $\mathscr{E}$ Biomimetics, vol. 9, no. 3, p. 036008, Sept. 2014. 
Quinn, D. B., Moored, K. W., Dewey, P. A., and Smits, A. J., "Unsteady propulsion near a solid boundary," Journal of Fluid Mechanics, vol. 742, pp. 152-170, Mar. 2014.

Rauworth, S. L., "Development of an underwater flapping foil tow-test system," Master's thesis, University of Rhode Island, 2014.

Read, D. A., "Oscillating foils for propulsion and maneuvering of ships and underwater vehicles," Master's thesis, Massachusetts Institute of Technology, 2001.

Rufo, M. and Smithers, M., "Ghostswimmer auv: Applying biomimetics to underwater robotics for achievement of tactical relevance," Marine Technology Society Journal, vol. 45, no. 4, 2011.

Ryu, J., Park, S. G., Kim, B., and Sung, H. J., "Flapping dynamics of a flexible propulsor near ground," Acta Mechanica Sinica, vol. 32, no. 6, pp. 991-1000, Dec. 2016.

Salumäe, T., Chemori, A., and Kruusmaa, M., "Motion control architecture of a 4-fin u-cat auv using dof prioritization," in Intelligent Robots and Systems (IROS), 2016 IEEE/RSJ International Conference on. IEEE, 2016, pp. 1321-1327.

Shen, J.-s., Zhou, X.-c., and Zhang, H.-g., "Research on Sliding Mode Control for NearSurface AUV Depth Regulation in Waves Circumstance," in 2010 International Conference on Electrical and Control Engineering. Wuhan, China: IEEE, June 2010, pp. $1760-1764$.

Siegenthaler, C., Pradalier, C., Gnther, F., Hitz, G., and Siegwart, R., "System integration and fin trajectory Design for a robotic sea-turtle," in 2013 IEEE/RSJ International Conference on Intelligent Robots and Systems, Nov. 2013, pp. 3790-3795.

Techet, A. H., "Propulsive performance of biologically inspired flapping foils at high Reynolds numbers," Journal of Experimental Biology, vol. 211, no. 2, pp. 274-279, Jan. 2008.

Vaccaro, R. J., Digital control: a state-space approach. McGraw-Hill New York, 1995, vol. 196.

Wu, J., Qiu, Y. L., Shu, C., and Zhao, N., "Pitching-motion-activated flapping foil near solid walls for power extraction: A numerical investigation," Physics of Fluids, vol. 26, no. 8, p. 083601, Aug. 2014.

Zhang, C., Huang, H., and Lu, X.-Y., "Free locomotion of a flexible plate near the ground," Physics of Fluids, vol. 29, no. 4, p. 041903, Apr. 2017. 